WHC-SP-1119

Copy No. 31

\title{
Radiological Control FY 1995 Site Support Program Plan WBS 6.7.2.4
}

Prepared for the U.S. Department of Energy

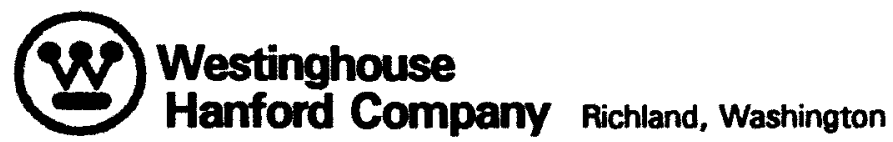

Hanford Operations and Engineering Contractor for the U.S. Depertment of Energy under Contract DE-AC06-87RL10930 


\section{Radiological Control FY 1995 Site Support Program Plan WBS 6.7.2.4}

Date Published

September 1994

Prepared for the U.S. Department of Energy Office of Environmental Restoration and Waste Management



Hanford Operations and Engineering Contractor for the

U.S. Department of Energy under Contract DE-AC06-87RL10930

Approved for Public Release

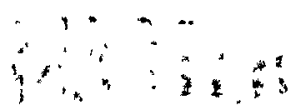




\section{LEGAL DISCLAIMER}

This report was prepared as an account of work sponsored by an agency of the United States Government Nelther the

United States Government nor any agency thereof, nor any of their employees nor any of their contractors subcontractors or their employees, makes any warranty, express or Implied or assumes any legal liability or responsiblity for the accuracy, completeness, or any third party's use or the results of such use of any information, apparatus, product, or process disclosed, or represents that its use would not infringe privately owned rights Reterence herein to any specific commercial product, process, or service by trade name. trademark, manufacturer, or otherwise, does not necessarlly constitute or imply its endorsement recommendation or favoring by the United States Government or any agency thereot or its contractors or subcontractors The views and opinions of authors expressed herein do not necessarly state or reflect those of the United States Government or any agency thereot

This report has been reproduced from the best avallable copy Avallable in paper copy and microfiche

Avallable to the U S Department of Energy

and its contractors from

Office of Scientific and Technical Information

PO Box 62

Oak Ridge, TN 37831

(615) $576-8401$

Avallable to the public from the U S Department of Commerce National Technical Information Service

5285 Port Royal Road

Springiteld, VA 22161

(703) $487-4650$

Printed in the United States of America

DISCLM 1 CHP (1 91) 


\section{DISCLAIMER}

Portions of this document may be illegible in electronic image products. Images are produced from the best available original document. 


\section{RELEASE AUTHORIZATION}

Document Number: WHC-SP-1119, REV 0

Document Title: Radiological Control FY 1995 Site Support Program PIan WBS 6.7 .2 .4

Release Date: $10 / 13 / 94$

$$
\text { * * * * * * * * * * * }
$$

This document was reviewed following the procedures described in WHC-CM-3-4 and is:

APPROVED FOR PUBLIC RELEASE

* * * * * * * * * * * *

WHC Information Release-Administration Specialist:

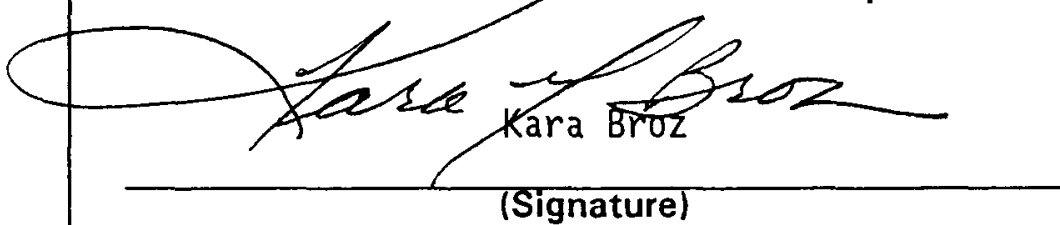

October 13,1994

(Signature)

(Date) 


\title{
Site Support Program Plan Approval Sheet
}

\author{
RADIOLOGICAL CONTROL - WBS \#6.7.2.4
}

Assistant Manager-Contracting Officer's Representative
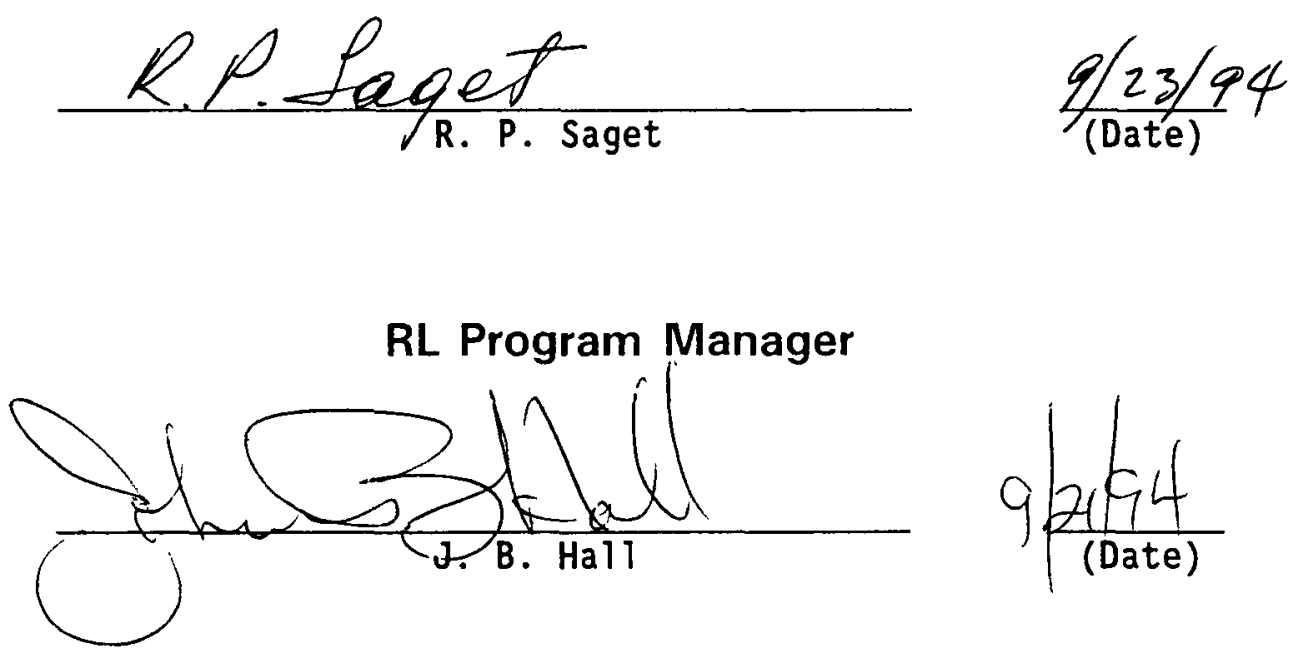

\section{WHC Director}

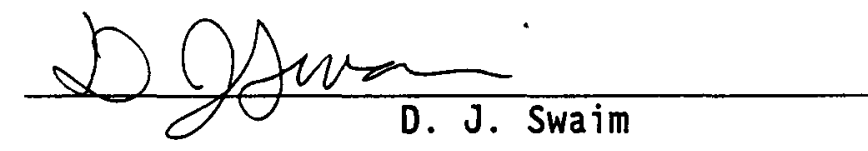

$\frac{9 / 23 / 94}{\text { (Date) }}$
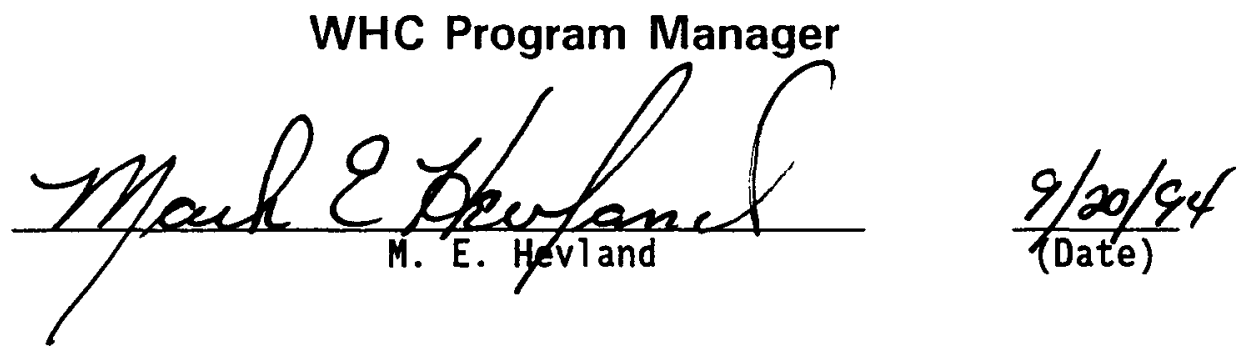


\section{5}

\section{RADIOLOGICAL CONTROL}

SITE SUPPORT PROGRAM PIAN

6.7 .2 .4

Radiological Control Program Control and Integration 


\section{Table of contents \\ for \\ 1995 Radiological Control Site Support Program Plan}

\section{Section 1 --Overview--}

Narrative of 1995 Site Support Program Plan...............1-1

Radiological Control and Hanford Vision.................

Radiological Control Mission Statement..................1-4

Radiological Control Objectives......................

Radiological Control Strategy.......................

Radiological Control Assumptions and Constraints...........1-7

Radiological Control Internal Needs Assessment.............1-8

Radiological Control External Needs Assessment.............1-9

Radiological Control Performance Indicators...............

\section{Section 2 --Baselines--}

Basis for Cost Estimates...........................

Cost Baseline by Program Element...................2-2

Full Time Equivalent Forecasts.....................

Description of Activities......................... $2-10$

Responsibility Assignment Matrix................... 


\section{Table of contents}

for

1995 Radiological Control Site Support Program Plan cont inued

\section{Section 3 --Work Breakdown Structure and Execution Year Work Plan}

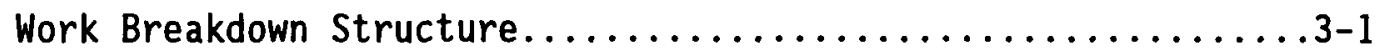

Work Breakdown Structure Dictionary Summary--Rad Con.........3-2

Radiological Control Management......................

Radiological Control Program Control and Integration.........3-6

Radiological Engineering and ALARA....................

Southern Areas and Support Services Radiological Control.....3-26

Dosimetry Pool Summary...........................30

Dosimetry Pool Work Breakdown Structure................3-31

Milestone List............................... 3-47

Milestone Description Sheets........................49

Section 4 --Appendix--

Direct Funded Radiological Control Activities..............4-1






\begin{tabular}{|c||c||c||}
\hline $\begin{array}{c}\text { Vision, Mission, } \\
\text { Introduction, Overview }\end{array}$ & $\begin{array}{c}\text { Westinghouse Hanford Company } \\
\text { Radiological Control } \\
6.7 .2 .4\end{array}$ & $\begin{array}{c}\text { Fr } 1995 \\
\text { Site Support Program P1an } \\
\text { August 30, 1994 }\end{array}$ \\
\hline
\end{tabular}

\section{Narrative of 1995 Site Support Program Plan}

The 1995 Site Support Program Plan (SSPP) represents a departure from past planning practices. It attempts to bring out year planning and execution year (EY) planning into a single document which will provide better understanding and continuity in planning. The plan consists of four sections:

\section{Section 1 Overview and Introduction}

Health Physics has been renamed to Radiological Control (RadCon). The role of RadCon is to protect workers, the public, and the environment from the harmful effects of radiation resulting from the DOE Hanford Site Operations. Section one expands this function in general terms and addresses issues that have an effect upon this mission. 


\begin{tabular}{|c||c||c||}
\hline $\begin{array}{c}\text { Vision, Mission, } \\
\text { Introduction, Overview }\end{array}$ & $\begin{array}{c}\text { Westinghouse Hanford Company } \\
\text { Radiological Control } \\
6.7 .2 .4\end{array}$ & $\begin{array}{c}\text { FY 1995 } \\
\text { Site Support Program Plan } \\
\text { August 30, 1994 }\end{array}$ \\
\hline
\end{tabular}

\section{Section 2 Cost Baselines which contains cost, technical and schedule baselines}

This reflects major changes in functional alignment as well as work breakdown structural alignment. The 1995 SSPP reflects these changes. The most noticeable change is in funding methods. Nearly all of the field functions have been funded directly by the projects and are carried in the projects program plans. This leaves the department overhead function with only those activities that require a company wide view point, such as:

- Providing a single point of contact for RadCon in the person of the RadCon Manager.

- Interpreting and guiding the implementation of DOE Orders, CFRs, and other regulations related to RadCon.

- Providing overall RadCon program planning.

- Administering the Radiological Problem Report and the Radiological Work Permit Systems.

- Overseeing the radiological records keeping system.

- Providing overall management of the Radiological Control Program.

These are best summed up in a few general areas which are represented by milestones in section 3 . These areas of oversight are:

- Coordinating the Implementation of 10 CFR 835.

- Reporting functions to DOE-RL.

- Performing self assessment, compliance, and lessons learned functions.

- Coordinating the Implementation of the Hanford Site Radiological Control Manual. 


\begin{tabular}{|c||c||c||}
\hline $\begin{array}{c}\text { Vision, Mission, } \\
\text { Introduction, Overview }\end{array}$ & $\begin{array}{c}\text { Westinghouse Hanford Company } \\
\text { Radiological Control } \\
6.7 .2 .4\end{array}$ & $\begin{array}{c}\text { Fy 1995 } \\
\text { Site Support Program Plan } \\
\text { August 30, 1994 }\end{array}$ \\
\hline
\end{tabular}

\section{Section 3 Execution Year Work Plan}

The execution year work $\mathrm{plan}$ is made up of cost summaries and detailed descriptions of the work to be done. The completion of the work is captured in milestones with a distinct completion date. This provides a schedule for the work to be accomplished. The required resources are captured in section three in Full Time Equivalent numbers and cost projections for the execution year of 1995.

\section{Section 4 Appendix}

The appendix to the SSPP includes important information not having a distinct place in the body of the plan.

The appendix includes a brief description of other project activities that are directly coupled to Radiological Control which are direct funded projects.

The methods used to establish the data in the SSPP were past experience base, Financial Data System escalation factors, and historical projections. The numbers reflected in the SSPP may not directly correlate with other planning efforts due to the different data used to make projections. As with any planning document, it is a plan and subject to change as it is put into use. The changes to the SSPP are controlled through a formal change control process. 


\begin{tabular}{|c||c||c||}
\hline $\begin{array}{c}\text { Vision, Mission, } \\
\text { Introduction, Overview }\end{array}$ & $\begin{array}{c}\text { Westinghouse Hanford Company } \\
\text { Radiological Control } \\
6.7 .2 .4\end{array}$ & $\begin{array}{c}\text { FY 1995 } \\
\text { Site Support Program Plan } \\
\text { August 30, 1994 }\end{array}$ \\
\hline
\end{tabular}

\section{Radiological Control and the Hanford Vision}

Together we are building a clean, accessible, and healthy environment which is part of a prospering and diversified community. We are reaching beyond our past achievements to continue a tradition of excellence in scientific and technological accomplishments. We are a resource that nations turn to for solutions to environmental challenges.

\section{Radiological Control Mission Statement}

The role of the RadCon program is to protect workers, the public, and the environment from the potentially harmful effects of exposure to radiation resulting from DOE's Hanford Site operations. The mission of Westinghouse Hanford Company RadCon organization as the administrator of the radiological control program, is to establish and maintain a high quality RadCon program and assist line management in its implementation. 


\begin{tabular}{|c||c||c||}
\hline $\begin{array}{c}\text { Vision, Mission, } \\
\text { Introduction, Overview }\end{array}$ & $\begin{array}{c}\text { Westinghouse Hanford Company } \\
\text { Radiological Control } \\
6.7 .2 .4\end{array}$ & $\begin{array}{c}\text { FY 1995 } \\
\text { Site Support Program P1an } \\
\text { August 30, 1994 }\end{array}$ \\
\hline
\end{tabular}

\section{Radiological Control Values}

- Establishing internal standards of accountability and integrity for each individual in Radiological Control.

- Establishing technically sound requirements which properly balance program cost, schedules, and technical excellence.

- Complying rigorously with requirements and procedures.

- Earning widespread respect as the leader, coach, mentor, and role model in the application of radiological controls.

- Becoming recognized by all our customers as an asset in accomplishing their missions.

- Establishing a strong safety culture such that safety becomes an integral part of every activity for each employee.

- Establishing strong labor/management relationships with a joint emphasis on the development of Total Quality leadership behaviors for all RadCon leaders.

- Utilizing the talents and skills of all RadCon staff for the enhancement and upgrading of the WHC radiological control program through employee involvement activities. 


\begin{tabular}{|c||c||c|c|}
\hline $\begin{array}{c}\text { Vision, Mission, } \\
\text { Introduction, Overview }\end{array}$ & $\begin{array}{c}\text { Nestinghouse Hanford Company } \\
\text { Radiological Control } \\
6.7 .2 .4\end{array}$ & $\begin{array}{c}\text { FY 1995 } \\
\text { Site Support Program Plan } \\
\text { August 30, 1994 }\end{array}$ \\
\hline
\end{tabular}

\section{Radiological Control Objectives}

The Radiological Control organization shall administer and control the Radiation Protection Program (RPP) for Westinghouse Hanford in a manner that is consistent with all applicable laws, regulations, and standards as defined by our customer. Radiological Control will provide a program that is risk based, efficient, and effective for all elements of radiological control.

\section{Radiological Control Strategy}

Radiological Control will accomplish this mission and objective by:

- Recruiting and training the best qualified personnel available.

- Encouraging professional development of all personnel.

- Analyzing and determining applicability of newly proposed rules and regulations.

- Incorporating applicable rules and regulations into the RPP.

- Planning the work and working the plan, with one plan for all of RadCon.

- Self-assessment of RadCon activities for quality, adequacy, performance, and methods for improvement.

- Accurately determining its resources and taking no work without adequate resources and prior planning.

- Those activities that are in support of direct funded programs will be controlled and funded directly by the direct program. RadCon will provide administrative, policy, and technical support as necessary.

- Developing and implementing a continuous long term improvement plan. 


\begin{tabular}{|c||c||c||}
\hline $\begin{array}{c}\text { Vision, Mission, } \\
\text { Introduction, Overview }\end{array}$ & $\begin{array}{c}\text { Westinghouse Hanford Company } \\
\text { Radiological Control } \\
6.7 .2 .4\end{array}$ & $\begin{array}{c}\text { FY 1995 } \\
\text { Site Support Program Plan } \\
\text { August 30, 1994 }\end{array}$ \\
\hline
\end{tabular}

\section{Radiological Control Assumptions and Constraints}

The following assumptions and constraints were made in the development of this SSPP.

- No new regulations more restrictive than 10 CFR 835 will be imposed.

- New technology will keep pace with continually reducing level of risk perception and the new technology will be funded.

- All milestone closures are assumed to be satisfactory if Radiological Control is not notified in writing by DOE-RL of any deficiencies.

- The restructuring within DOE and WHC has made usable forecasting of costs and personnel past 1995 very difficult. New baselines must be established before cost, skills mix, or total FTEs can be forecast. These constraints lead to the assumption that this plan is accurate in so far as there are no changes from present conditions or activities. All changes shall be promulgated through the formally approved change control process. 


\begin{tabular}{|c||c||c||}
\hline $\begin{array}{c}\text { Vision, Mission, } \\
\text { Introduction, Overview }\end{array}$ & $\begin{array}{c}\text { Westinghouse Hanford Company } \\
\text { Radiological Control } \\
6.7 .2 .4\end{array}$ & $\begin{array}{c}\text { FY 1995 } \\
\text { Site Support Program Plan } \\
\text { August 30, 1994 }\end{array}$ \\
\hline
\end{tabular}

\section{Internal Needs Assessment Summary}

Radiological Control products include radiological technical services, dosimetry monitoring, regulatory compliance, and Health Physics Technicians (HPTs) to provide radiation protection support of program, operations, and maintenance of facilities and project.

It is the responsibility of Radiological Control to be aware of and protect the needs of the environment and general public as well as radiological workers at the Hanford Site. This effort significantly adds to the confidence level of the general public.

It is expected that new technology and increased alertness on the part of the public and various watch dog groups will significantly impact future costs.

As the site moves towards more remediation activities, monitoring costs and record keeping costs will increase. Levels of risk and exposure to radiological hazards may be elevated. In general Radiological Control has the personnel and capacity to handle these new challenges, however an active program for training or recruiting new management personnel may be necessary. 


\begin{tabular}{|c||c||c||}
\hline $\begin{array}{c}\text { Vision, Mission, } \\
\text { Introduction, Overview }\end{array}$ & $\begin{array}{c}\text { Westinghouse Hanford Company } \\
\text { Radiological Control } \\
6.7 .2 .4\end{array}$ & $\begin{array}{c}\text { FY 1995 } \\
\text { Site Support Program Plan } \\
\text { August 30, 1994 }\end{array}$ \\
\hline
\end{tabular}

\section{Radiological Control External Needs Summary}

Radiological Control provides four products to its customers. HPTs are recruited and trained for radiation protection support of facility and Program operations and maintenance. The Radiological Engineering group of Radiological Control provides a variety of technical services for the various facilities on radiological issues. Program Control and Integration provides assessment of regulatory compliance and policy implementation for a safe radiological protection program. The dosimetry personnel provide exposure monitoring for internal and external exposure. This provides a baseline for maintaining exposures ALARA for all Westinghouse radiological workers and visitors.

\section{Radiological Control Performance Indicators}

These Performance Indicators provide details for the execution year work plan. The successful completion of the RadCon yearly mission and work plan will be measured by comparison to the following baselines.

- Completion of DOE-RL Milestones on or before scheduled completion date.

- Internal self assessments as determined by the RadCon Program, HSRCM, and 10 CFR 835.

- Completion of work scope and milestones within cost guidelines specified in this plan.

- Internal tracking of these indicators will be used to ensure timely actions and necessary corrections to mandate completions of scheduled milestones on time and with in cost estimates. 


\begin{tabular}{|c|c|c|}
\hline Cost Baseline & $\begin{array}{c}\text { Westinghouse Hanford Company } \\
\text { Radiological Control } \\
6.7 .2 .4 \\
\end{array}$ & $\begin{array}{c}\text { FY } 1995 \text { SSPP } \\
\text { Date Prepared: } \\
\text { August } 30,1994 \\
\end{array}$ \\
\hline
\end{tabular}

Basis for Cost Estimates:

The cost baseline which is included in this SSPP is based upon the estimated 1995 costs. The out years are projected using an escalation factor of 3 percent for 1996 and 4 percent for each out year. Radiological control will continue to identify more cost effective methods for performing work activities and combined with outsourcing will result in reduced out year staffing levels.

The restructuring of WHC Radiological Control and the SSPP process has made it difficult to compare data from the 1994 and previous years to the 1995 and subsequent year costs. Therefore the 1994 costs are not included. 


\begin{tabular}{|c|c|c|}
\hline & Westinghouse Hanford Company & FY 1995 SSPP \\
2.C.1 Cost Baseline & Radiological Control Prepared: \\
by Program Element & 6.7 .2 .4$. & August 30, 1994: \\
\hline
\end{tabular}

FY 1994 Cost Baseline (Dollars in Thousands)

\begin{tabular}{|l|c|c|}
\hline WBS \# & Title & Total \$s \\
\hline & & \\
\hline & [Excludes company adders (G\&A and CSP/Oversight)] FY 1994 Total for (6.7.2.4) & \\
\hline
\end{tabular}

FY 1995 Cost Baseline (Dollars in Thousands)

\begin{tabular}{|l|l|l|}
\hline 6.7 .2 .4 & & \\
\hline 6.7 .2 .4 .1 & RadCon Manager and Administration & $\$ 5,244.6$ \\
\hline 6.7 .2 .4 .1 .1 & RadCon Program Control and Integration & $\$ 1,215.7$ \\
\hline 6.7 .2 .4 .2 & Radiological Engineering and ALARA & $\$ 1,400.3$ \\
\hline 6.7.2.4.2.1 & Dosimetry Pool & $\$ 12,743.5$ \\
\hline 6.7.2.4.4.1 & RadCon Field Activities & $\$ 354.7$ \\
\hline & [Excludes company adders (G\&A and CSP/Oversight)] FY 1995 Total for (6.7.2.4) & $\$ 20,958.8$ \\
\hline
\end{tabular}




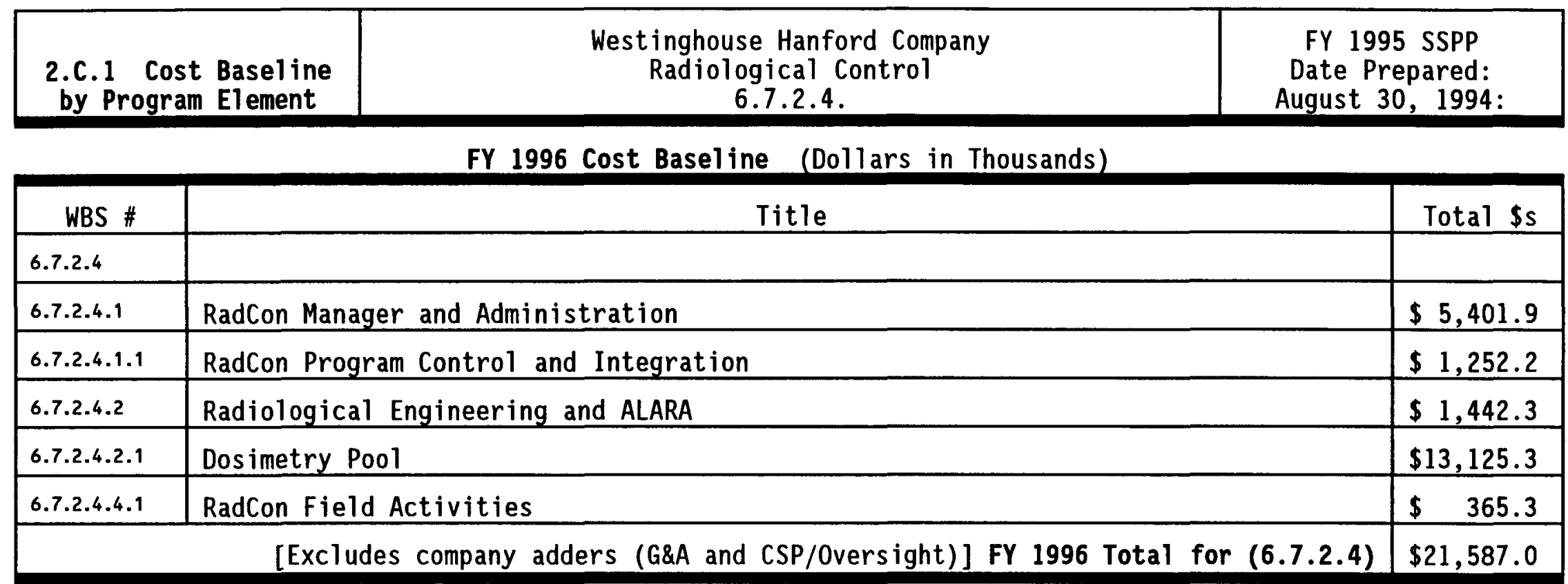

FY 1997 Cost Baseline (Dollars in Thousands)

\begin{tabular}{|l|l|l|}
\hline 6.7 .2 .4 & & \\
\hline 6.7 .2 .4 .1 & RadCon Manager and Administration & $\$ 5,618.0$ \\
\hline 6.7.2.4.1.1 & RadCon Program Control and Integration & $\$ 1,302.3$ \\
\hline 6.7.2.4.2 & Radiological Engineering and ALARA & $\$ 1,500.0$ \\
\hline 6.7.2.4.2.1 & Dosimetry Pool & $\$ 13,650.3$ \\
\hline 6.7.2.4.4.1 & RadCon Field Activities & $\$ 380.0$ \\
\hline & [Excludes company adders (G\&A and CSP/Oversight)] FY 1997 Total for (6.7.2.4) & $\$ 22,450.6$ \\
\hline
\end{tabular}




\begin{tabular}{|c|c|c|c|}
\hline $\begin{array}{l}\text { 2.C. } 1 \text { Cost Baseline } \\
\text { by Program El ement }\end{array}$ & $\begin{array}{c}\text { West inghouse Hanford Company } \\
\text { Radiological Control } \\
6.7 .2 .4 \text {. }\end{array}$ & \multicolumn{2}{|c|}{$\begin{array}{c}\text { FY } 1995 \text { SSPP } \\
\text { Date Prepared: } \\
\text { August } 30,1994:\end{array}$} \\
\hline & FY 1998 Cost Baseline (Dollars in The & & \\
\hline WBS \# & \multicolumn{2}{|l|}{ Title } & Total \$s \\
\hline \multicolumn{4}{|l|}{ 6.7.2.4 } \\
\hline 6.7.2.4.1 & \multicolumn{2}{|l|}{ RadCon Manager and Administration } & $\$ 5,842.7$ \\
\hline 6.7.2.4.1.1 & \multicolumn{2}{|l|}{ RadCon Program Control and Integration } & $\$ 1,354.3$ \\
\hline 6.7 .2 .4 .2 & \multicolumn{2}{|l|}{ Radiological Engineering and ALARA } & $\$ 1,560.0$ \\
\hline 6.7.2.4.2.1 & \multicolumn{2}{|l|}{ Dosimetry Pool } & $\$ 14,196.3$ \\
\hline 6.7.2.4.4.1 & \multicolumn{2}{|l|}{ RadCon Field Activities } & $\$ \quad 395.2$ \\
\hline \multicolumn{3}{|c|}{ [Excludes company adders (G\&A and CSP/Oversight)] FY 1998 Total for (6.7.2.4) } & $\$ 23,348.5$ \\
\hline
\end{tabular}

FY 1999 Cost Baseline (Dollars in Thousands)

\begin{tabular}{|l|l|l|}
\hline 6.7 .2 .4 & & \\
\hline 6.7 .2 .4 .1 & RadCon Manager and Administration & $\$ 6,076.4$ \\
\hline 6.7 .2 .4 .1 .1 & RadCon Program Control and Integration & $\$ 1,408.5$ \\
\hline 6.7 .2 .4 .2 & Radiological Engineering and ALARA & $\$ 1,622.4$ \\
\hline 6.7 .2 .4 .2 .1 & Dosimetry Pool & $\$ 14,764.2$ \\
\hline 6.7 .2 .4 .4 .1 & RadCon Field Activities & $\$ 11.0$ \\
\hline & [Excludes company adders (G\&A and CSP/Oversight)] FY 1999 Total for (6.7.2.4) & $\$ 24,282.5$ \\
\hline
\end{tabular}




\begin{tabular}{|c|c|c|}
\hline & Westinghouse Hanford Company & FY 1995 SSPP \\
2.C.1 Cost Baseline & Radiological Control & Date Prepared: \\
by Program Element & 6.7 .2 .4 & August 30, 1994: \\
\hline
\end{tabular}

FY 2000 Cost Baseline (Dollars in Thousands)

\begin{tabular}{|l|l|l|}
\hline \multicolumn{1}{|c|}{ WBS\# } & \multicolumn{1}{|c|}{ Title } & Total \$s \\
\hline 6.7.2.4 & & \\
\hline 6.7.2.4.1 & RadCon Manager and Administration & $\$ 6,319.5$ \\
\hline 6.7.2.4.1.1 & RadCon Program Control and Integration & $\$ 1,464.9$ \\
\hline 6.7.2.4.2 & Radiological Engineering and ALARA & $\$ 1,687.3$ \\
\hline 6.7.2.4.2.1 & Dosimetry Pool & $\$ 15,354.7$ \\
\hline 6.7.2.4.4.1 & RadCon Field Activities & $\$ 227.4$ \\
\hline & [Excludes company adders (G\&A and CSP/Oversight)] FY 2000 Total for (6.7.2.4) & $\$ 25,253.8$ \\
\hline
\end{tabular}

FY 2001 Cost Baseline (Dollars in Thousands)

\begin{tabular}{|l|l|l|}
\hline 6.7.2.4 & RadCon Manager and Administration & \\
\hline 6.7.2.4.1 & RadCon Program Control and Integration & $\$ 6,572.3$ \\
\hline 6.7.2.4.1.1 & Radiological Engineering and ALARA & $\$ 1,523.5$ \\
\hline 6.7.2.4.2 & Dosimetry Pool & $\$ 1,754.8$ \\
\hline 6.7.2.4.4.1 & RadCon Field Activities & $\$ 15,968.9$ \\
\hline & [Excludes company adders (G\&A and CSP/Oversight)] FY 2001 Total for (6.7.2.4) & $\$ 26,264.0$ \\
\hline
\end{tabular}




\begin{tabular}{|c|c}
\hline FTE Forecasts & $\begin{array}{c}\text { Westinghouse Hanford Company } \\
\text { Radiological Control } \\
6.7 .2 .4\end{array}$ \\
\hline
\end{tabular}

JOB FAMILY - Ful1 Time Equivalent Staff by Job Description

\begin{tabular}{|l|l|l|l|}
\hline Job category & 1994 & 1995 & 1996 \\
\hline
\end{tabular}

\section{MAMAGERS}

First line

General/executive

Project/Program

other

Subtotal Managers

ENGINEERS

Chemical

civil

Computer Software

Electrical

Environmental

Industrial

Mechanical

Nuclear

Plant

Qual ity Control

\begin{tabular}{|r|r|r|r|}
\hline 34 & 6 & & \\
\hline 12 & 3 & & \\
\hline 2 & 2 & & \\
\hline
\end{tabular}

FY 1995

Site Support Program Plan Date Prepared: August 30, 1994

NOTE: Job Family Only After 1996

\begin{tabular}{|l|l|l}
\hline 1999 & 2000 & 2001
\end{tabular}

1998

\begin{tabular}{|c|c|c|c|c|c|c|c|}
\hline 34 & 6 & & & & & & \\
\hline 12 & 3 & & & & & & \\
\hline 2 & 2 & & & & & & \\
\hline 2 & & & & & & & \\
\hline 50 & 11 & 11 & 11 & 11 & 11 & 11 & 11 \\
\hline
\end{tabular}

\begin{tabular}{|l|l|}
\hline & \\
\hline & \\
\hline & \\
\hline & \\
\hline
\end{tabular}

\begin{tabular}{|l|l|l|l|}
\hline & & \\
\hline & & & \\
\hline & & & \\
\hline & & & \\
\hline & & & \\
\hline & & & \\
\hline
\end{tabular}




\begin{tabular}{|c|c|c|c|c|c|c|c|c|}
\hline FTE Forecasts & \multicolumn{4}{|c|}{$\begin{array}{c}\text { West inghouse Hanford Company } \\
\text { Radiological Control } \\
6.7 .2 .4\end{array}$} & & \multicolumn{3}{|c|}{$\begin{array}{c}\text { FY } 1995 \\
\text { Site Support Program Plan } \\
\text { Date Prepared: August } 30,1994\end{array}$} \\
\hline \multicolumn{9}{|c|}{ JOB FAMILY - Full Time Equivalent Staff by Job Description } \\
\hline Job category & 1994 & 1995 & 1996 & 1997 & 1998 & 1999 & 2000 & 2001 \\
\hline \multicolumn{9}{|l|}{ Safety } \\
\hline \multicolumn{9}{|l|}{ other } \\
\hline Subtotal Engineers & 6 & 5 & 5 & 5 & 5 & 5 & 5 & 5 \\
\hline \multicolumn{9}{|l|}{ SCIENTISTS } \\
\hline \multicolumn{9}{|l|}{ Chemists } \\
\hline \multicolumn{9}{|l|}{ Environmental } \\
\hline \multicolumn{9}{|l|}{ Life } \\
\hline \multicolumn{9}{|l|}{ Mathematicians } \\
\hline Physicists Heal th & 28 & 15 & 15 & & & & & \\
\hline \multicolumn{9}{|l|}{ Social Industrial Hygienist } \\
\hline \multicolumn{9}{|l|}{ other } \\
\hline Subtotal Scientists & 28 & 15 & 15 & 15 & 15 & 15 & 15 & 15 \\
\hline \multicolumn{9}{|l|}{ ADMIN/OTHER PROFESSIONALS } \\
\hline \multicolumn{9}{|l|}{ Accountant/audi tor } \\
\hline \multicolumn{9}{|l|}{ Cormunications Specialist } \\
\hline \multicolumn{9}{|l|}{ Compliance inspectors } \\
\hline Computer System Anal & 1 & & & & & & & \\
\hline \multicolumn{9}{|l|}{ Cost Est/planner/sch } \\
\hline Heal th Physics Analyst & 39 & 6 & 6 & & & & & \\
\hline Industrial Hygiene & & & & & & & & \\
\hline
\end{tabular}

The FTEs reflected in this table are averages and may not reflect the actual number of persons employed at any given time. Out year numbers are estimates based upon current statfing and expecting little change after fiscal year 1995 . 


\begin{tabular}{|c|c|c|c|c|c|c|c|c|}
\hline FTE Forecasts & \multicolumn{4}{|c|}{$\begin{array}{c}\text { Westinghouse Hanford Company } \\
\text { Radiological Control } \\
6.7 .2 .4\end{array}$} & & \multicolumn{3}{|c|}{$\begin{array}{c}\text { FY } 1995 \\
\text { Site Support Program Plan } \\
\text { Date Prepared: August } 30,1994\end{array}$} \\
\hline \multicolumn{9}{|c|}{ JOB FAMILY - Ful1 Time Equivalent Staff by Job Description } \\
\hline Job category & 1994 & 1995 & 1996 & 1997 & 1998 & 1999 & 2000 & 2001 \\
\hline \multicolumn{9}{|l|}{ Safeguard \& Security } \\
\hline \multicolumn{9}{|l|}{ Trainers } \\
\hline Staff Asst \& Records Specialists & 15 & & & & & & & \\
\hline Subtotal Admin/Other Professionals & 55 & 6 & 6 & 6 & 6 & 6 & 6 & 6 \\
\hline \multicolumn{9}{|l|}{ GEN ADM/SECRETARY/CLERK } \\
\hline Admin Assistants & 19 & 10 & 10 & & & & & \\
\hline Office Clerks (Gen) & 8 & & & & & & & \\
\hline office Clerks (Special) & 2 & 7 & 7 & & & & & \\
\hline Secretaries & 14 & 8 & 8 & & & & & \\
\hline \multicolumn{9}{|l|}{ Typist/Word Process } \\
\hline \multicolumn{9}{|l|}{ other } \\
\hline Subtotal Gen Adm/Secretary/Clerk & 43 & 25 & 25 & 25 & 25 & 25 & 25 & 25 \\
\hline \multicolumn{9}{|l|}{ TECHNICIANS } \\
\hline \multicolumn{9}{|l|}{ Computer Oper/Coder } \\
\hline \multicolumn{9}{|l|}{ Engrs/Tech } \\
\hline \multicolumn{9}{|l|}{ Envir. Sci Technicians } \\
\hline Heal th Phys. Technic. & 358 & 37 & 37 & & & & & \\
\hline \multicolumn{9}{|l|}{ Indus. Saf/Heal th Tech } \\
\hline \multicolumn{9}{|l|}{ Instru/Control Tech } \\
\hline \multicolumn{9}{|l|}{ Other } \\
\hline Subtotal Technicians & 358 & 37 & 37 & 37 & 37 & 37 & 37 & 37 \\
\hline
\end{tabular}

The FTEs reflected in this table are averages and may not reflect the actual number of persons employed at any given time. Out year numbers are estimates based upon current staffing and expecting little change after fiscal year 1995. 


\begin{tabular}{|c|c|c|c|c|c|c|c|c|}
\hline FTE Forecasts & \multicolumn{4}{|c|}{$\begin{array}{c}\text { Westinghouse Hanford Company } \\
\text { Radiological Control } \\
6.7 .2 .4\end{array}$} & & \multicolumn{3}{|c|}{$\begin{array}{l}\text { FY } 1995 \\
\text { Site Support Program Plan } \\
\text { Date Prepared: August 30, } 1994\end{array}$} \\
\hline \multicolumn{9}{|c|}{ JOB FAMILY - Full Time Equivalent Staff by Job Description } \\
\hline Job category & 1994 & 1995 & 1996 & 1997 & 1998 & 1999 & 2000 & 2001 \\
\hline \multicolumn{9}{|l|}{ CRAFTS } \\
\hline \multicolumn{9}{|l|}{ Electricians } \\
\hline \multicolumn{9}{|l|}{ other } \\
\hline \multicolumn{9}{|l|}{ Instrument Specialist } \\
\hline \multicolumn{9}{|l|}{ Locksmith Safemaster } \\
\hline Subtotal Crafts & 0 & 0 & 0 & & & & & \\
\hline \multicolumn{9}{|l|}{ LABOR \& GEN HORKERS } \\
\hline \multicolumn{9}{|l|}{ Firefighters } \\
\hline \multicolumn{9}{|l|}{ Security Guards } \\
\hline \multicolumn{9}{|l|}{ other } \\
\hline \multicolumn{9}{|l|}{ Paramedics } \\
\hline Subtotal Labor \& Gen Workers & 0 & 0 & 0 & & & & & \\
\hline Total FTEs 6.7 .2 .4 & 540 & 99 & 99 & 99 & 99 & 99 & 99 & 99 \\
\hline
\end{tabular}

94 staff includes Environmental Restoration Contractor and direct funded staff.

95 staff reflects non-aligned staff only. 


\begin{tabular}{|c|c|c|}
\hline $\begin{array}{l}\text { 2.A.2 Description } \\
\text { of Activities }\end{array}$ & $\begin{array}{r}\text { Westinghouse H } \\
\text { Radiologic } \\
6.7\end{array}$ & $\begin{array}{l}\text { FY } 1995 \\
\text { Site Support Program Plan } \\
\text { Prepared: Aug 30, } 1994 \\
\end{array}$ \\
\hline Activity No. & Activity Title & Description \\
\hline 6.7 .2 .4 .1 & Radiological Control Management & $\begin{array}{l}\text { Manage and direct the total Radiological Control } \\
\text { Program for Westinghouse Hanford Company. Provides } \\
\text { service as the Radiological Control Manager (RCM) } \\
\text { for all WHC Activities. }\end{array}$ \\
\hline 6.7 .2 .4 .1 .1 & RC Program Control and Integration & $\begin{array}{l}\text { Activities include: records management, storage, and } \\
\text { disposition; self assessment and lessons learned } \\
\text { communication process administration; manpower } \\
\text { planning and staffing; HAMTC contract negotiations, } \\
\text { grievance process, and leadership council; RPR and } \\
\text { RWP program administration; Organizational } \\
\text { performance indicators and Administrative plans and } \\
\text { reports, write and monitor the Radiological } \\
\text { Protection Program (RPP), provide technical } \\
\text { interface in the area of radiation protection, } \\
\text { provide administrative support, track and trend RC } \\
\text { data, provide RC Planning and support of the SMS and } \\
\text { the SSPP, analyze and coordinate implementation of } \\
\text { the requirements of DoE/EH-0256T and } 10 \text { CFR 835, } \\
\text { provide commitment tracking and trending, compliance } \\
\text { self assessment, Radiological Work Permit } \\
\text { administration, and Radiological Control } \\
\text { communications enhancement. }\end{array}$ \\
\hline 6.7 .2 .4 .2 & Radiological Engineering and ALARA & $\begin{array}{l}\text { Activities include: developing radiological work } \\
\text { practices; providing radiological control } \\
\text { instrumentation support; monitoring and } \\
\text { administering the source control program; and } \\
\text { providing technical support as required in all areas } \\
\text { of Radiological Control. }\end{array}$ \\
\hline 6.7 .2 .4 .2 .1 & Dosimetry & $\begin{array}{l}\text { Administer the WHC Dosimetry program (See Section } 3 \\
\text { for details). }\end{array}$ \\
\hline 6.7 .2 .4 .2 .2 & Radiological Control Technology & $\begin{array}{l}\text { Administer the Source Control Program and the Site } \\
\text { Cognizant Engineer Program. }\end{array}$ \\
\hline
\end{tabular}




\begin{tabular}{|c|c|c|}
\hline $\begin{array}{l}\text { 2.A.2 Description } \\
\text { of Activities }\end{array}$ & $\begin{array}{c}\text { Westinghouse Hanford } \\
\text { Radiological Cont } \\
6.7 .2 .4 \\
\end{array}$ & $\begin{array}{l}\text { FY } 1995 \\
\text { Site Support Program Plan } \\
\text { Prepared: Aug 30, } 1994\end{array}$ \\
\hline Activity No. & Activity Title & Description \\
\hline 6.7 .2 .4 .2 .3 & Radiological Engineering & $\begin{array}{l}\text { Provide necessary Radiological Control Procedures } \\
\text { and work practices as required. }\end{array}$ \\
\hline 6.7 .2 .4 .2 .4 & $\begin{array}{l}\text { Radiological ALARA and Contamination } \\
\text { Control Project }\end{array}$ & $\begin{array}{l}\text { Activities include: Assist line management and sub- } \\
\text { contractors with implementation of the radiological } \\
\text { control program and ALARA work practices; Administer } \\
\text { the Contamination Control Improvement Project; track } \\
\text { and trend contamination issues; and provide maps of } \\
\text { contaminated areas. }\end{array}$ \\
\hline 6.7 .2 .4 .4 .1 & $\begin{array}{l}\text { Field Support Activities--Overhead Funded } \\
\text { portion of Southern Area and RC Support } \\
\text { Services only }\end{array}$ & $\begin{array}{l}\text { Activities include: providing supervisory leadership } \\
\text { and management; engineering support; project } \\
\text { planning and oversight; radiation and contamination } \\
\text { exposure control; performing special and routine } \\
\text { radiological surveys, air sampling, and complete } \\
\text { legal documentation of conditions; monitor } \\
\text { facilities, materials, equipment, and employees for } \\
\text { radioactive contamination. }\end{array}$ \\
\hline
\end{tabular}




\begin{tabular}{|c|c|c|c|c|c|}
\hline \multicolumn{2}{|c|}{$\begin{array}{c}2 . A .1 \\
\text { WBS \&esponsibility } \\
\text { Assignment } \\
\text { Matrix }\end{array}$} & \multicolumn{2}{|r|}{$\begin{array}{c}\text { Westinghouse Hanford Company } \\
\text { Radiological Control } \\
6.7 .2 .4\end{array}$} & \multicolumn{2}{|c|}{$\begin{array}{l}\text { FY } 1995 \\
\text { Site Support Program Plan } \\
\text { Date Prepared: August 30, } 1994\end{array}$} \\
\hline $\begin{array}{l}\text { Activity } \\
\text { No. }\end{array}$ & $\begin{array}{l}\text { Cost } \\
\text { Account }\end{array}$ & $\begin{array}{r}\text { WBS Work } \\
\text { Package } \\
\end{array}$ & Title & $\begin{array}{l}\text { Responsible } \\
\text { Manager }\end{array}$ & $\begin{array}{l}\text { Responsible } \\
\text { Organization }\end{array}$ \\
\hline 6.7 .2 .4 .1 & $\mathrm{~J} 330 \mathrm{~A}$ & 6.7 .2 .4 .1 & RadCon Management \& Administration & ME Hevland & 33000 \\
\hline 6.7 .2 .4 .1 .1 & $\mathrm{~J} 33 \mathrm{AB}$ & 6.7 .2 .4 .1 .1 & RadCon Prog. Control and Integration & SR Johnson & 33800 \\
\hline 6.7 .2 .4 .1 .1 & J33A8 & 6.7 .2 .4 .1 .1 & Radiological Control Program Manual Support & SR Johnson & 33800 \\
\hline 6.7 .2 .4 .1 .1 & $\mathrm{~J} 33 \mathrm{AB}$ & 6.7 .2 .4 .1 .1 & Program Improvement Initiatives & SR Johnson & 33800 \\
\hline 6.7 .2 .4 .1 .1 & J33A8 & 6.7 .2 .4 .1 .1 & $\begin{array}{l}\text { Technical Support for Implementation of } \\
10 \text { CFR } 835\end{array}$ & SR Johnson & 33800 \\
\hline 6.7 .2 .4 .1 .1 & $\mathrm{~J} 33 \mathrm{~A} A 8$ & 6.7 .2 .4 .1 .1 & $\begin{array}{l}\text { Implementation of Hanford Site Radiological } \\
\text { Control Manual }\end{array}$ & SR Johnson & 33800 \\
\hline 6.7 .2 .4 .1 .1 & J33A8 & 6.7 .2 .4 .1 .1 & $\begin{array}{l}\text { Radiological Control (RC) Records } \\
\text { Management }\end{array}$ & SR Johnson & 33800 \\
\hline 6.7 .2 .4 .1 .1 & $\mathrm{~J} 33 \mathrm{~A} 8$ & 6.7 .2 .4 .1 .1 & RC Staff Services, Planning \& Recruitment & SR Johnson & 33800 \\
\hline 6.7 .2 .4 .1 .1 & J33A8 & 6.7 .2 .4 .1 .1 & Radiation Work Permit Administration & SR Johnson & 33800 \\
\hline 6.7 .2 .4 .1 .1 & J33A8 & 6.7 .2 .4 .1 .1 & Radiological Problem Report Administration & SR Johnson & 33800 \\
\hline 6.7 .2 .4 .1 .1 & J33A8 & 6.7 .2 .4 .1 .1 & Self Assessment/Lessons Learned Programs & SR Johnson & 33800 \\
\hline 6.7 .2 .4 .1 .1 & J33A8 & 6.7 .2 .4 .1 .1 & General Administrative Support Activities & SR Johnson & 33800 \\
\hline 6.7 .2 .4 .1 .1 & J33A8 & 6.7 .2 .4 .1 .1 & $\begin{array}{l}\text { Radiological Control Site Support Program } \\
\text { Planning }\end{array}$ & SR Johnson & 33800 \\
\hline 6.7 .2 .4 .2 & $\mathrm{~J} 3360$ & 6.7 .2 .4 .2 & Radiological Engineering and ALARA & TO Merkling & 33600 \\
\hline 6.7 .2 .4 .2 .1 & $1 M D C O A$ & 6.7 .2 .4 .2 .1 & Dosimetry & WA Decker & 33600 \\
\hline
\end{tabular}




\begin{tabular}{|c|c|c|c|c|c|}
\hline \multicolumn{2}{|c|}{$\begin{array}{c}2 . A .1 \\
\text { WBS \& Responsibility } \\
\text { Assignment } \\
\text { Matrix }\end{array}$} & \multicolumn{2}{|r|}{$\begin{array}{c}\text { Westinghouse Hanford Company } \\
\text { Radiological Control } \\
6.7 .2 .4\end{array}$} & \multicolumn{2}{|c|}{$\begin{array}{c}\text { FY } 1995 \\
\text { Site Support Program Plan } \\
\text { Date Prepared: August 30, } 1994\end{array}$} \\
\hline $\begin{array}{l}\text { Activity } \\
\text { No. }\end{array}$ & $\begin{array}{l}\text { Cost } \\
\text { Account }\end{array}$ & $\begin{array}{r}\text { WBS Work } \\
\text { Package }\end{array}$ & Title & $\begin{array}{l}\text { Responsible } \\
\text { Manager }\end{array}$ & $\begin{array}{l}\text { Responsible } \\
\text { Organization }\end{array}$ \\
\hline 6.7 .2 .4 .2 .1 & 1MDCOAM200 MDPNL & 6.7 .2 .4 .2 .1 .1 & Portable Instrumentation and Calibration & TD Merkling & 33600 \\
\hline 6.7 .2 .4 .2 .1 & 1MDCOAM101 M111A & 6.7 .2 .4 .2 .1 .2 & Oversee PNL dosimetry contract to WHC & UA Decker & 33600 \\
\hline 6.7 .2 .4 .2 .1 & 1MDCOAM103 M111C & 6.7 .2 .4 .2 .1 .3 & $\begin{array}{l}\text { Workplace Air Sampling and Respiratory } \\
\text { Protection Programs }\end{array}$ & WA Decker & 33600 \\
\hline 6.7 .2 .4 .2 .1 & 1MDCOAM103 M1110 & 6.7 .2 .4 .2 .1 .4 & Access Control Entry System (ACES) & HA Decker & 33600 \\
\hline 6.7 .2 .4 .2 .1 & 1MOCOAM103 M111E & 6.7 .2 .4 .2 .1 .5 & $\begin{array}{l}\text { Dosimetry Management, Administration and } \\
\text { Support }\end{array}$ & WA Decker & 33600 \\
\hline 6.7 .2 .4 .2 .1 & 1MDCOAM103 M111F & 6.7 .2 .4 .2 .1 .6 & IRM Support of ACES Program & WA Decker & 33600 \\
\hline 6.7 .2 .4 .2 .1 & 1MOCOAM109 M111H & 6.7 .2 .4 .2 .1 .7 & $\begin{array}{l}\text { ALARA and Contamination Control Improvement } \\
\text { Project }\end{array}$ & TD Merkling & 33600 \\
\hline 6.7 .2 .4 .2 .1 & 1MDCOAM110 M111J & 6.7 .2 .4 .2 .1 .8 & Payment to PNL for HSRCM Support & SR Johnson & 33000 \\
\hline 6.7 .2 .4 .2 & 1J330A0601 J3360 & 6.7 .2 .4 .2 & Implement Technology Improvements & TD Merkling & 33600 \\
\hline 6.7.2.4.2. & $1 \mathrm{~J} 330 \mathrm{A0601} \mathrm{J} 3360$ & 6.7 .2 .4 .2 & Radiological Cognizant Engineering & TD Merkling & 33600 \\
\hline 6.7 .2 .4 .2 & $1 \mathrm{~J} 330 \mathrm{A0601} \mathrm{J} 3360$ & 6.7 .2 .4 .2 & Radiological Control Procedures & TD Merkling & 33600 \\
\hline 6.7 .2 .4 .2 & 1J330A0601 J3360 & 6.7 .2 .4 .2 & Source Control Program & RM Rogers & 33600 \\
\hline 6.7 .2 .4 .4 .1 & $1 \mathrm{J330A0100}$ & 6.7 .2 .4 .4 .1 & $\begin{array}{l}\text { Radiological Control Support for Southern } \\
\text { Areas and Support Services Radiological } \\
\text { Control -- includes only overhead funded } \\
\text { portion }\end{array}$ & RL watts & 33100 \\
\hline
\end{tabular}




\section{RADIOLOGICAL CONTROL WORK BREAK DOWN STRUCTURE FOR FY95}

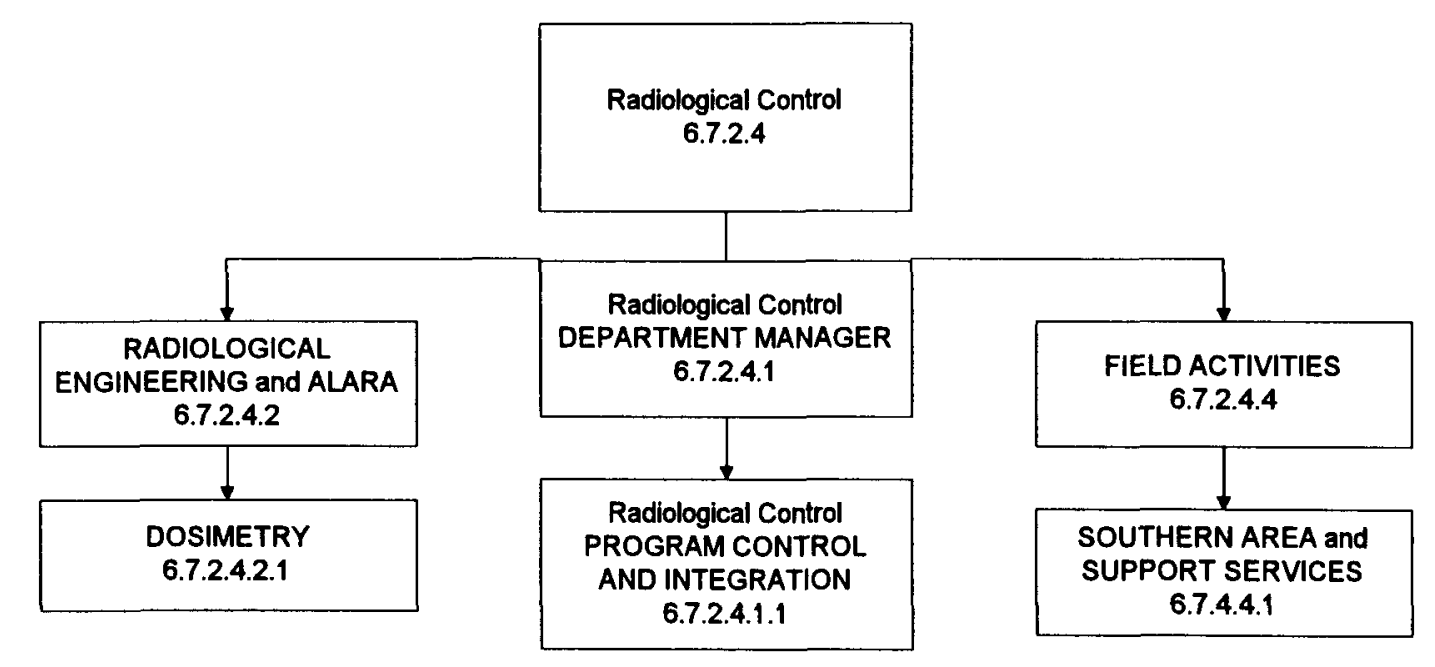




\begin{tabular}{|c|c|c|c|c|c|}
\hline \multirow{6}{*}{$\begin{array}{l}\text { Work } \\
\text { Breakdown } \\
\text { Structure } \\
\text { Dictionary }\end{array}$} & \multirow{6}{*}{\multicolumn{3}{|c|}{$\begin{array}{c}\text { Westinghouse Hanford Company } \\
\text { HEALTH PHYSICS } \\
\text { Part I - Summary } \\
\text { (DOLLARS IN 000'S) }\end{array}$}} & \multirow{8}{*}{$\begin{array}{l}\text { FY } 1995 \text { SSPP } \\
\text { Rev. \# } 0\end{array}$} & \multirow{3}{*}{\begin{tabular}{|l|} 
Funding Source: \\
G\&A \\
SWS \\
\end{tabular}} \\
\hline & & & & & \\
\hline & & & & & \\
\hline & & & & & OST \\
\hline & & & & & $\mathrm{DOH} X \mathrm{X}$ \\
\hline & & & & & MGT PAO \\
\hline Cost Account & \multirow{2}{*}{\multicolumn{3}{|c|}{$\begin{array}{l}\text { Cost Account Title } \\
\text { HEALTH PHYSICS OVERHEAD }\end{array}$}} & & POOL \\
\hline $1 \mathrm{~J} 33$ & & & & & DIRECT \\
\hline $\begin{array}{l}\text { SMS WBS } \\
6724\end{array}$ & \multicolumn{3}{|c|}{$\begin{array}{l}\text { SMS Title } \\
\text { RADIOLGGICAO CONTROL OVERHEAD }\end{array}$} & \multirow{2}{*}{\multicolumn{2}{|c|}{$\begin{array}{c}\text { Annuallzed Rate } \\
\text { (For Organizational Overhead and } \\
\text { Rated Service Pool Use Only) }\end{array}$}} \\
\hline \multicolumn{2}{|l|}{ CAM Review/Approval } & ME HEVLAND & $27 / 94$ & & \\
\hline \multirow{2}{*}{\multicolumn{2}{|c|}{$\begin{array}{l}\text { SMS Program Manager Review/Approv: } \\
\text { Financlal Manager Review/Approvat }\end{array}$}} & ME HEVLAND & Date & FY 1994 Rate & FY 1995 Rate Request \\
\hline & & L.C. BRANDT & Date $9-27-94$ & $38 \%$ & $32 \%$ \\
\hline \multicolumn{2}{|l|}{ Responsible Analyst } & A.W. SPILLMAN & Date $9 / 27 / 94$ & FY 1995 Target Rate & FY 1995 Approved Rate \\
\hline \multicolumn{3}{|l|}{ OSBRB Review/Approval } & Date & & \\
\hline \multirow[b]{2}{*}{ FULL-TIME EQUIVALENTS (FTES) } & \multicolumn{2}{|c|}{ FY1994 } & \multicolumn{3}{|c|}{ FY 1995} \\
\hline & Budget & $\begin{array}{c}\text { Fiscal Year Spending } \\
\text { Forecast (FYSF) }\end{array}$ & Target & Request & $\begin{array}{l}\text { Approved } \\
\text { Baseline }\end{array}$ \\
\hline Organizational - Exempt & 429 & 324 & & 28.3 & \\
\hline Organizational - Nonexempt & 112 & 249 & & 6.5 & \\
\hline Organizational - Bargaining & 54 & 56 & & 1.2 & \\
\hline Total Organizational FTEs & 595 & 62.9 & & 36 & \\
\hline Suppon FTEs & 184 & 179 & & 1.2 & \\
\hline TOTALFTES & 779 & 808 & & 37.2 & \\
\hline \multicolumn{6}{|l|}{ COST ELEMENTS } \\
\hline Labor - Regular & 4.510 & 4,634 & \multirow{12}{*}{ 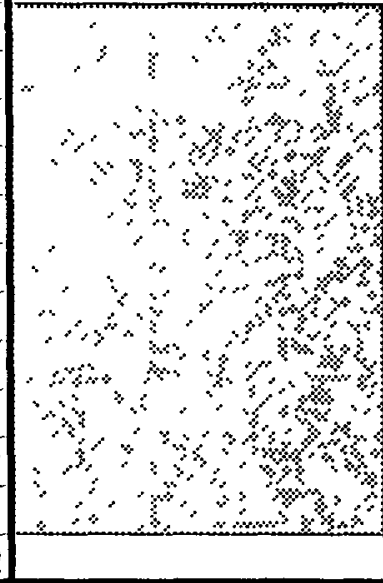 } & 2,139 & \multirow{12}{*}{$\begin{array}{l}3 \\
\% \\
8\end{array}$} \\
\hline Labor - Overtime & 46 & 46 & & 5 & \\
\hline 0 Total Labor & 4,556 & 4,680 & & 2,144 & \\
\hline 1 Materials & 283 & 278 & & 353 & \\
\hline 2 Purchased Services & 321 & 284 & & 887 & \\
\hline 3 Other Hanford & 4 & 5 & & 4 & \\
\hline 4 Site Services & 1,630 & 1,653 & & 1,680 & \\
\hline 5 Internal Charges & 2,700 & 2,700 & & 2,583 & \\
\hline 6 IRM Support & 705 & 735 & & 658 & \\
\hline 7 Overheads & 532 & 477 & & 6 & \\
\hline \multicolumn{3}{|l|}{8 Revenue } & & & \\
\hline TOTAL DOLLARS & 10,730 & 10,812 & & 8,313 & \\
\hline
\end{tabular}




\begin{tabular}{|c|c|c|c|c|c|c|c|c|c|c|c|c|c|c|c|c|c|}
\hline $\begin{array}{l}\text { Work } \\
\text { Breakdown } \\
\text { Structure } \\
\text { Dictionary }\end{array}$ & \multicolumn{14}{|c|}{$\begin{array}{l}\text { Westinghouse Hanford Company } \\
\text { HEALTH PHYSICS } \\
\text { Part III - Liquidation Base Analysis } \\
\text { ORGANIZATIONAL OVERHEAD ONLY }\end{array}$} & \multicolumn{3}{|c|}{$\begin{array}{l}\text { FY } 1995 \text { SSPP } \\
\text { Revision \# } 0\end{array}$} \\
\hline $\begin{array}{l}\text { Cost Account } \\
1 \mathrm{~J} 33\end{array}$ & \multicolumn{17}{|c|}{$\begin{array}{l}\text { Cost Account Title } \\
\text { HEALTH PHYSICS OVERHEAD }\end{array}$} \\
\hline & Ff 9994 & & & & & & a 19 & & & & $\because$ & & 8 & f(a) & 6 & $x=10$ & \#.tat \\
\hline DIRECT STAFF* & $\begin{array}{c}\text { FOAECAST } \\
(\$ 000) \\
\quad\end{array}$ & OCT & Nov & DEC & JAN & FEB & MAR & APR & MAY & JUN & JUL & AUG & SEP & $\begin{array}{c}\text { AVG. } \\
\text { DIRECT } \\
\text { STAFF }\end{array}$ & $\begin{array}{c}\text { AVG } \\
\text { LABOR } \\
\text { RATE }\end{array}$ & $\begin{array}{c}\text { REALIZATION } \\
\text { HOURS }\end{array}$ & $\begin{array}{l}\text { DIRECT } \\
\text { LABOR } \\
(\$ 000)\end{array}$ \\
\hline EXEMPT & \multirow{5}{*}{$\begin{array}{l}3 \\
8 \cdots \\
8\end{array}$} & 106 & 106 & 106 & 106 & 106 & 106 & 106 & 106 & 106 & 106 & 106 & 106 & 106 & 35.97 & 1812 & 6,909 \\
\hline NONEX / PT / TEMP & & 21 & 21 & 21 & 21 & 21 & 21 & 21 & 21 & 21 & 21 & 21 & 21 & 21 & 14.47 & 1812 & 551 \\
\hline $\mathrm{B} / \mathrm{U}(\mathrm{HPT})$ & & 312 & 312 & 312 & 312 & 312 & 312 & 312 & 312 & 312 & 312 & 312 & 312 & 312 & 29.40 & 1812 & 16,621 \\
\hline OVERTIME FACTOR \# & & $3:$ & & & & $2 x$ & $\infty$ & & & & $\$$ & B.\%? & 2 & 233 & 3 & $\%$ & 2,475 \\
\hline DIRECT LABOR BASE & & 439 & 439 & 439 & 439 & 439 & 439 & 439 & 439 & 439 & 439 & 439 & 439 & 439 & $8 \%$ & 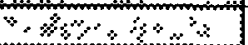 & 26,005 \\
\hline
\end{tabular}

\begin{tabular}{|c|c|c|c|}
\hline ORG OVHD COST & 10,812 & 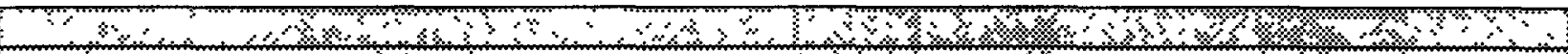 & 8,313 \\
\hline DIRECT LABOR BASE @ & 28,752 & $\%<0^{\circ}<<4 \% \diamond$ & 26,005 \\
\hline (COST/BASE) & $37.6 \%$ & ২ค४ & $32.0 \%$ \\
\hline
\end{tabular}

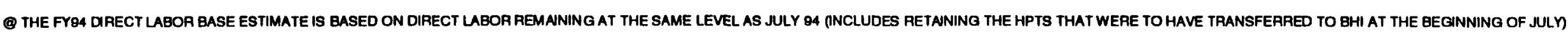
- FY94 OVERTME IS ESTIMATED TO BE \$3,300 FY95 OVERTIME IS ESTIMATED TO BE 75\% OF FY94. \$2,475

- Direct staff is stafi that charges babor to char ge codes on which organizational overtiead ls aRolled 


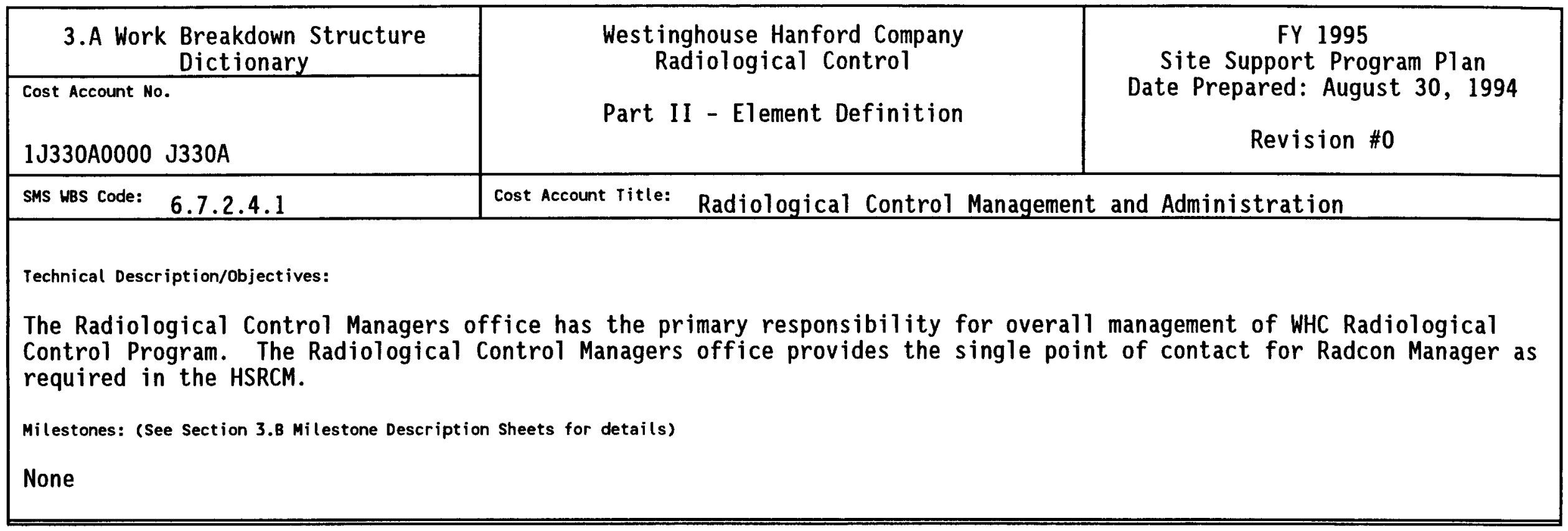




\begin{tabular}{|c|c|c|c|c|c|c|c|c|c|c|}
\hline \multirow{3}{*}{$\begin{array}{l}\text { Cost Account No. } 1 \mathrm{~J} 330 \mathrm{~A} 0000 \mathrm{~J} 330 \mathrm{~A} \\
\text { Element Task Description } \\
\text { Requests Within Target: }\end{array}$} & \multicolumn{10}{|c|}{ Part II - Radiological Control Management \& Administration - } \\
\hline & \multicolumn{2}{|c|}{1994 FYSF } & \multicolumn{2}{|c|}{ Delta } & \multicolumn{2}{|c|}{ FY 1995} & \multicolumn{2}{|c|}{ Delta } & \multicolumn{2}{|c|}{ FY 1996} \\
\hline & FTEs & $\$ \mathbf{s}$ & FTES & $\$ \mathbf{s}$ & FTEs & $\$ \mathbf{s}$ & FTES & \$s & FTES & $\$ s$ \\
\hline \multicolumn{11}{|l|}{$\begin{array}{l}\text { Hork Scope Detailed Description: } \\
\text { Overall management of Radiological Control }\end{array}$} \\
\hline WHC Labor Cost & 3.5 & 175.0 & & & 2.8 & 139.9 & & & 2.8 & 144.1 \\
\hline Total Labor & 3.5 & 175.0 & & & 2.8 & 139.9 & & & 2.8 & 144.1 \\
\hline Total Labor Contracts & & & & & & & & & & \\
\hline \multicolumn{11}{|l|}{$\begin{array}{l}\text { (List ONLY items that exceed \$5K. [i.e., } 20 \text { Computers]) } \\
\text { Non-Labor Cost Descriptions }\end{array}$} \\
\hline \multicolumn{11}{|l|}{$\begin{array}{l}\text { Non-Labor costs including occupancy costs, } \\
\text { management reserve, P.0 Labor, materials and misc. } \\
\text { costs. }\end{array}$} \\
\hline Total Non-Labor & & & & & & 5104.7 & & & & 5257.8 \\
\hline Total Cost Account Within Target & 3.5 & 175.0 & & & 2.8 & 5244.6 & & & 2.8 & 5401.9 \\
\hline
\end{tabular}




\begin{tabular}{|l|c|c|}
\hline 3.A Work Breakdown Structure & Westinghouse Hanford Company \\
Dictionary & Radiological Control & $\begin{array}{c}\text { FY 1995 } \\
\text { Site Support Program P1an } \\
\text { Dugust 30, 1994 }\end{array}$ \\
\hline Cost Account No. & Part II - Element Definition & Revared: \\
IJ330A0800 J33A8 & & Revision \#0 \\
\hline
\end{tabular}

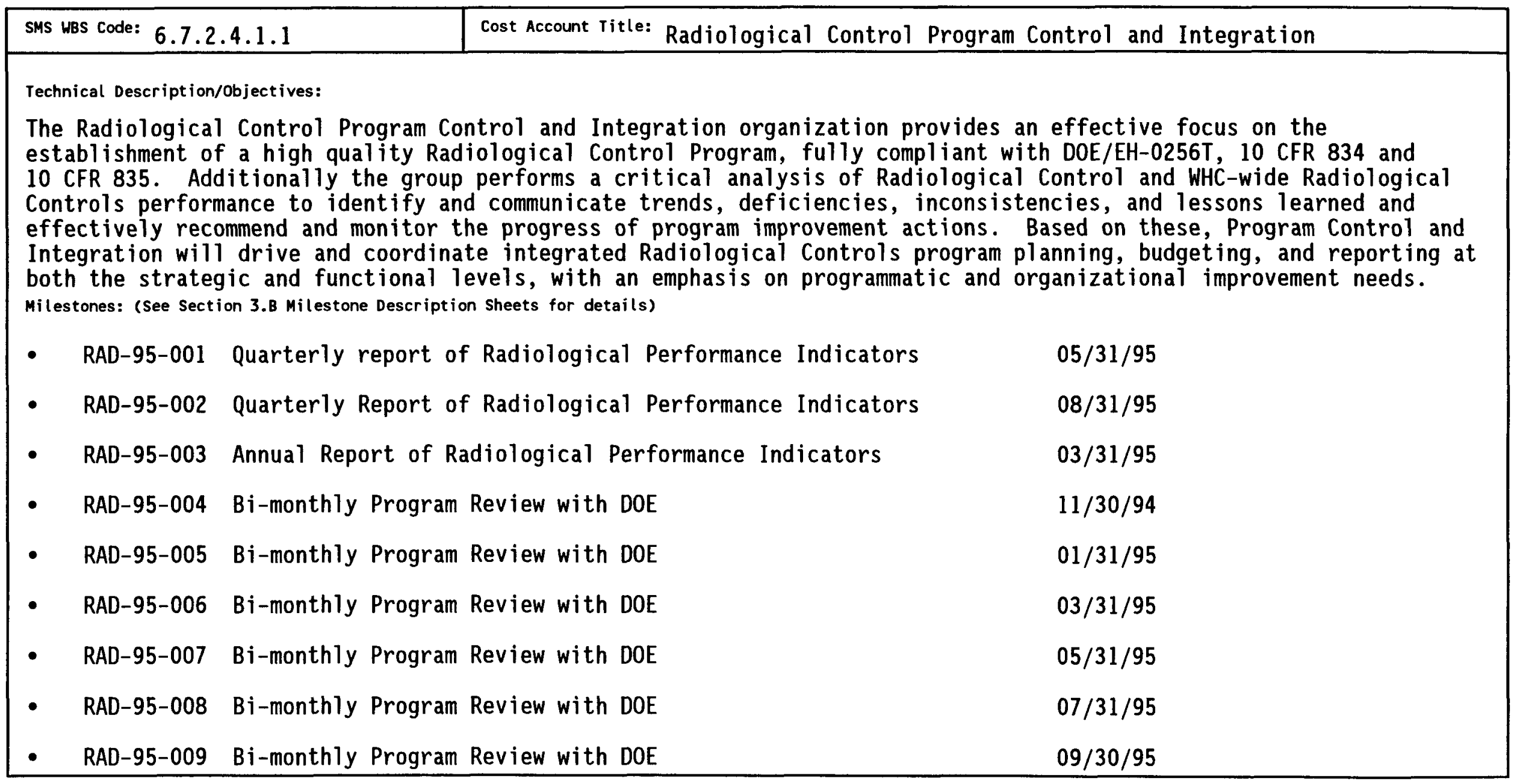




\begin{tabular}{|c|c|c|}
\hline $\begin{array}{c}\text { 3. A Work Breakdown Structure } \\
\text { Dictionary }\end{array}$ & $\begin{array}{l}\text { Westinghouse Hanford Company } \\
\text { Radiological Control }\end{array}$ & $\begin{array}{c}\text { FY } 1995 \\
\text { Site Support Program Plan }\end{array}$ \\
\hline $\begin{array}{l}\text { Cost Account No. } \\
1 \mathrm{~J} 330 \mathrm{~A} 0800 \quad \mathrm{~J} 33 \mathrm{~A} 8\end{array}$ & Part II - Element Definition & $\begin{array}{l}\text { Date Prepared: August 30, } 1994 \\
\text { Revision \#0 }\end{array}$ \\
\hline
\end{tabular}

\begin{tabular}{|c|c|c|}
\hline SMS WBS Code: 6.7 .2 .4 .1 .1 & \multicolumn{2}{|c|}{ Cost Account Title: Radiological Control Program Control and Integration } \\
\hline \multicolumn{3}{|l|}{ Milestones continued } \\
\hline - $\quad$ RAD-95-016 & Submittal of RPP to DOE & $12 / 31 / 94$ \\
\hline - $\quad$ RAD-95-017 & Year end report of DOE RadCon Manual Compliance & $12 / 31 / 94$ \\
\hline - $\quad$ RAD-95-018 & Report of Status of HSRCM Rev 1.0 Compliance Actions & $09 / 30 / 95$ \\
\hline - $\quad$ RAD-95-019 & Complete all actions for Rev. 0 of the DOE RadCon Manual & $03 / 31 / 95$ \\
\hline - $\quad$ RAD-95-022 & Area Emergency Preparedness Drills & $03 / 31 / 95$ \\
\hline - $\quad$ RAD-95-023 & Area Emergency Preparedness Drills & $09 / 30 / 95$ \\
\hline - $\quad$ RAD-95-024 & Area Emergency Preparedness Drills & $12 / 31 / 94$ \\
\hline RAD-95-025 & Area Emergency Preparedness Drills & $06 / 30 / 95$ \\
\hline
\end{tabular}




\begin{tabular}{|c|c|c|c|c|c|c|c|c|c|c|}
\hline Cost Account No. $1 \mathrm{~J} 330 \mathrm{~A} 0800 \mathrm{~J} 33 \mathrm{~A} 8$ & \multicolumn{10}{|c|}{ Part II - Management \& Administration - Radiological Control } \\
\hline Element Task Description & \multicolumn{2}{|c|}{1994 FYSF } & \multicolumn{2}{|c|}{ Delta } & \multicolumn{2}{|c|}{ FY 1995} & \multicolumn{2}{|c|}{ Delta } & \multicolumn{2}{|c|}{ FY 1996} \\
\hline Requests Within Target: & FTES & $\$ s$ & FTEs & $\$ s$ & FTES & $\$ s$ & FTEs & $\$ s$ & FTES & $\$ s$ \\
\hline $\begin{array}{l}\text { Work Scope Detailed Description: } \\
\begin{array}{l}\text { Manage and direct the Radiological Control Program } \\
\text { Control and Integration Group. } \\
\text { - PCI Manager position } \\
\text { - PCI Group Secretary }\end{array}\end{array}$ & & & & & & & & & & \\
\hline WHC Labor Cost & 2.0 & 92.0 & & & 2.0 & 92.0 & & & 2.0 & 94.8 \\
\hline
\end{tabular}




\begin{tabular}{|c|c|c|c|c|c|c|c|c|c|c|}
\hline \multirow{3}{*}{$\begin{array}{l}\text { Cost Account No. 1J330A0800 J33A8 } \\
\text { Element Task Description } \\
\text { Requests Within Target: }\end{array}$} & \multicolumn{10}{|c|}{ Part II - Management \& Administration - Radiological Control } \\
\hline & \multicolumn{2}{|c|}{1994 FYSF } & \multicolumn{2}{|c|}{ Delta } & \multicolumn{2}{|c|}{ FY 1995} & \multicolumn{2}{|c|}{ Delta } & \multicolumn{2}{|c|}{ FY 1996} \\
\hline & FTES & $\$ s$ & FTES & $\$ \mathbf{s}$ & FTES & $\$ s$ & FTES & $\$ s$ & FTES & $\$ s$ \\
\hline $\begin{array}{l}\text { Hork Scope Detailed Descriptions: } \\
\text { Maintain an effective process for determining and } \\
\text { reporting WHC status on implementation of the Hanford } \\
\text { Site Radiological Control Manual (HSRCM), including } \\
\text { quarterly status reports to WHC senior management and } \\
\text { DOE-RL on specific or general problems/issues in } \\
\text { implementation, and recommendations for improvement } \\
\text { actions. } \\
\text { - Provide weekly status reports to all } \\
\text { Radiological Control Managers, a monthly status } \\
\text { report to all WHC Directors and Plant Managers } \\
\text { and a quarterly report to DOE-RL. } \\
\text { Maintain a commitment tracking system to track } \\
\text { implementation actions for the HSRCM. Maintain } \\
\text { files of documentation for closure. } \\
\text { - Provide briefings/training to Management and WHC } \\
\text { employees as requested to aid in elevating the } \\
\text { line workers general knowledge on the HSRCM } \\
\text { implementation actions. }\end{array}$ & & & & & & & & & & \\
\hline
\end{tabular}




\begin{tabular}{|c|c|c|c|c|c|c|c|c|c|c|}
\hline Cost Account No. $1 \mathrm{~J} 330 \mathrm{~A} 0800 \mathrm{~J} 33 \mathrm{AB}$ & \multicolumn{10}{|c|}{ Part II - Management \& Administration - Radiological Control } \\
\hline Element Task Description & \multicolumn{2}{|c|}{1994 FYSF } & \multicolumn{2}{|c|}{ Delta } & \multicolumn{2}{|c|}{ FY 1995} & \multicolumn{2}{|c|}{ Delta } & \multicolumn{2}{|c|}{ FY 1996} \\
\hline Requests Within Target: & FTEs & $\$$ s & FTES & $\$ s$ & FTES & $\$ s$ & FTES & \$s & FTEs & $\$ \mathbf{s}$ \\
\hline 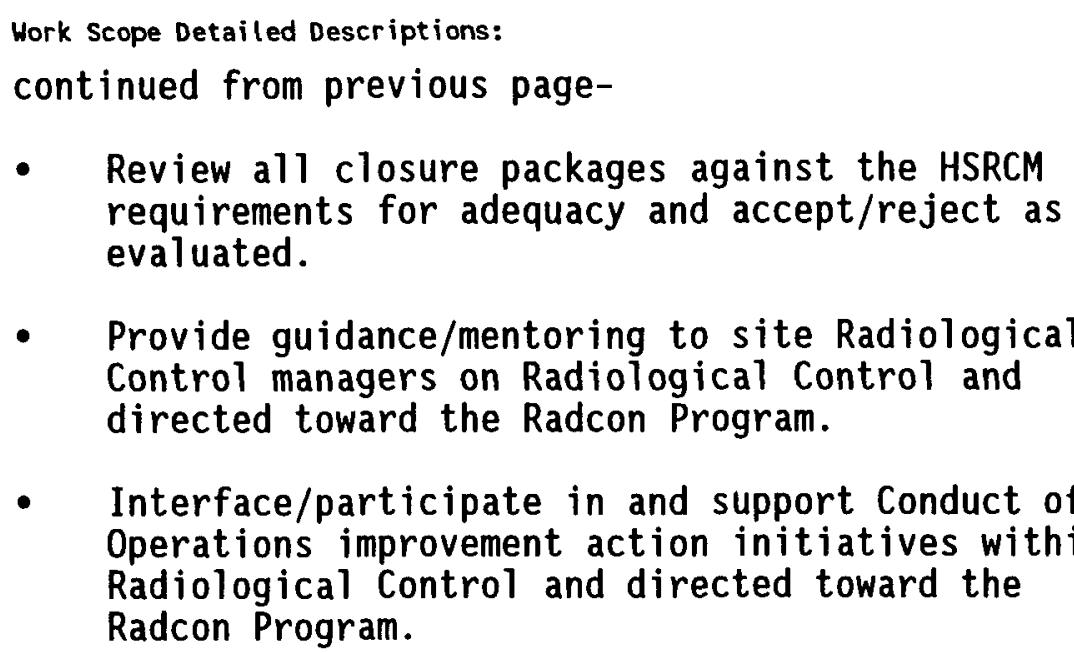 & & & & & & & & & & \\
\hline WHC LABOR COSTS & 1.0 & 65.0 & & & 1.0 & 65.0 & & & 1.0 & 65.0 \\
\hline
\end{tabular}




\begin{tabular}{|c|c|c|c|c|c|c|c|c|c|c|}
\hline \multirow{3}{*}{$\begin{array}{l}\text { Cost Account No. } 1 \mathrm{~J} 330 \mathrm{~A} 0800 \mathrm{~J} 33 \mathrm{AB} \\
\text { Element Task Description } \\
\text { Requests Within Target: }\end{array}$} & \multicolumn{10}{|c|}{ Part II - Management \& Administration - Radiological Control } \\
\hline & \multicolumn{2}{|c|}{1994 FYSF } & \multicolumn{2}{|c|}{ Delta } & \multicolumn{2}{|c|}{ FY 1995} & \multicolumn{2}{|c|}{ Delta } & \multicolumn{2}{|c|}{ FY 1996} \\
\hline & FTES & \$s & FTES & $\$$ s & FTES & \$s & FTES & $\$ s$ & FTES & $\$ s$ \\
\hline $\begin{array}{l}\text { Work Scope Detailed Descriptions: } \\
\text { Coordinate with all WHC facilities the development of } \\
\text { an updated implementation plan and schedule for } \\
\text { DOE/EH-0256T, which takes into account WHC progress } \\
\text { and problems in implementing DOE/EH-0256T, as well as } \\
\text { new developments since submittal of the original } \\
\text { plan. } \\
\text { - Respond to facility specific problems with } \\
\text { interpretations and implementation actions for } \\
\text { manual requirements as requested by facility } \\
\text { representatives. Provide technical/policy } \\
\text { guidance for issues. } \\
\text { Coordinate weekly meetings with the Points of } \\
\text { Contact from each facility to foster teamwork } \\
\text { between facilities on implementation activities } \\
\text { for the HSRCM. } \\
\text { Provide WHC single point of contact in support } \\
\text { of development and maintenance of the HSRCM. } \\
\text { Review practices, procedures, position papers, } \\
\text { and other requirements implementing instructions } \\
\text { against HSRCM requirements. }\end{array}$ & & & & & & & & & & \\
\hline WHC LABOR COSTS & 2.7 & 175.5 & & & 1.7 & 110.5 & & & 1.7 & 113.9 \\
\hline
\end{tabular}




\begin{tabular}{|c|c|c|c|c|c|c|c|c|c|c|}
\hline \multirow{3}{*}{$\begin{array}{l}\text { Cost Account No. 1J330A0800 J33A8 } \\
\text { Element Task Description } \\
\text { Requests Within Target: }\end{array}$} & \multicolumn{10}{|c|}{ Part II - Management \& Administration - Radiological Control } \\
\hline & \multicolumn{2}{|c|}{1994 FYSF } & \multicolumn{2}{|c|}{ Delta } & \multicolumn{2}{|c|}{ FY 1995} & \multicolumn{2}{|c|}{ Delta } & \multicolumn{2}{|c|}{ FY 1996} \\
\hline & FTEs & $\$ s$ & FTEs & \$s & FTES & $\$ s$ & FTEs & $\$ s$ & FTEs & $\$ s$ \\
\hline $\begin{array}{l}\text { Work Scope Detailed Descriptions: } \\
\text { Develop and Coordinate the implementation of an } \\
\text { effective, company-wide strategy and plan for } \\
\text { responding to the issuance of } 10 \text { CFR } 835 \text { and the } \\
\text { occupational radiological protection program } \\
\text { provisions of } 10 \text { CFR } 834 \text {. The plan should be oriented } \\
\text { toward driving quantum improvements in discipl ined } \\
\text { compliance with company requirements and procedures } \\
\text { which implement these regulations, achieving a high } \\
\text { degree of excellence in program-level compliance with } \\
\text { regulations, and limiting WHC liability to the } \\
\text { minimum required by law. } \\
\text { - Coordinate weekly POC meetings to review the } \\
\text { draft RPP and incorporate facility specific } \\
\text { concerns and practices. } \\
\text { Develop, submit for approval, update and } \\
\text { maintain the WHC } 10 \text { CFR } 835 \text { RPP. } \\
\text { Coordinate with General Councils office and } \\
\text { other WHC functional organizations developing } \\
\text { implementation for PAAA regulations. } \\
\text { Identify, develop, and submit } \\
\text { exemption/deviation requests for } 10 \text { CFR } 835 \\
\text { implementation issues. }\end{array}$ & & & & & & & & & & \\
\hline
\end{tabular}




\begin{tabular}{|c|c|c|c|c|c|c|c|c|c|c|}
\hline \multirow{3}{*}{$\begin{array}{l}\text { Cost Account No. } 1 \mathrm{~J} 330 \mathrm{~A} 0800 \mathrm{~J} 33 \mathrm{~A} 8 \\
\text { Element Task Description } \\
\text { Requests Within Target: }\end{array}$} & \multicolumn{10}{|c|}{ Part II - Management \& Administration - Radiological Control } \\
\hline & \multicolumn{2}{|c|}{1994 FYSF } & \multicolumn{2}{|c|}{ Delta } & \multicolumn{2}{|c|}{ FY 1995} & \multicolumn{2}{|c|}{ Delta } & \multicolumn{2}{|c|}{ FY 1996} \\
\hline & FTES & $\$$ s & FTES & $\$ s$ & FTES & $\$ s$ & FTES & $\$ s$ & FTES & $\$ s$ \\
\hline $\begin{array}{l}\text { Hork Scope Detailed Descriptions: } \\
\text { cont inued from previous page- } \\
\text { - Track } 10 \text { CFR } 835 \text { implementation actions. } \\
\text { - Interface with DOE-RL and DOE-HQ staff on } \\
\text { PAAA/835 issues. Provide input to DOE on } \\
\text { infrastructure development for Nuclear Safety } \\
\text { Regulatory/Enforcement process as solicited by } \\
\text { DOE. } \\
\text { - Coordinate Radiological Control Response to } \\
\text { Notice of Violations and Enforcement issues. } \\
\text { Develop and track to completion new milestones } \\
\text { as necessary to support } 10 \text { CFR } 835 \text { compliance } \\
\text { actions that are beyond the scope of existing } \\
\text { RCM milestone. }\end{array}$ & & & & & & & & & & \\
\hline WHC LABOR COSTS & 1.2 & 78.0 & & & 1.2 & 78.0 & & & 1.2 & 80.3 \\
\hline
\end{tabular}




\begin{tabular}{|c|c|c|c|c|c|c|c|c|c|c|}
\hline \multirow{3}{*}{$\begin{array}{l}\text { Cost Account No. 1J330A0800 J33A8 } \\
\text { Element Task Description } \\
\text { Requests Within Target: }\end{array}$} & \multicolumn{10}{|c|}{ Part II - Management \& Administration - Radiological Control } \\
\hline & \multicolumn{2}{|c|}{1994 FYSF } & \multicolumn{2}{|c|}{ Delta } & \multicolumn{2}{|c|}{ FY 1995} & \multicolumn{2}{|c|}{ Delta } & \multicolumn{2}{|c|}{ FY 1996} \\
\hline & FTEs & $\$ s$ & FTEs & $\$ s$ & FTEs & $\$ s$ & FTEs & $\$ s$ & FTES & $\$ s$ \\
\hline $\begin{array}{l}\text { Work scope Detailed Descriptions: } \\
\text { Develop and implement a low-impact/high-value self } \\
\text { assessment and internal review process for the } \\
\text { Radiological Control organization. The program shall } \\
\text { provide for continuing, critical assessment of both } \\
\text { Radiological Control organizational performance and } \\
\text { WHC's Radcon performance. } \\
\text { - Establish and coordinate a Radiological Control } \\
\text { and WHC Radcon self assessment program as a } \\
\text { positive value oriented process, fully } \\
\text { applicable to RadCon activities at WHC's Hanford } \\
\text { facilities and programs, worth seeking after } \\
\text { with a long term cooperative commitment between } \\
\text { Hanford employees of all job classifications; } \\
\text { Maintain a tracking system to monitor } \\
\text { improvement actions of deficiencies identified } \\
\text { through the self assessment process. } \\
\text { Maintain documentation files to provide } \\
\text { compliance status of the WHC RadCon program at } \\
\text { all WHC facilities. } \\
\text { Provide periodic independent assessment of the } \\
\text { Radiological Control and WHC RadCon self } \\
\text { assessment process. }\end{array}$ & & & & & & & & & & \\
\hline
\end{tabular}




\begin{tabular}{|c|c|c|c|c|c|c|c|c|c|c|}
\hline \multirow{3}{*}{$\begin{array}{l}\text { Cost Account No. 1J330A0800 J33A8 } \\
\text { Element Task Description } \\
\text { Requests Within Target: }\end{array}$} & \multicolumn{10}{|c|}{ Part II - Management \& Administration - Radiological Control } \\
\hline & \multicolumn{2}{|c|}{1994 FYSF } & \multicolumn{2}{|c|}{ Delta } & \multicolumn{2}{|c|}{ FY 1995} & \multicolumn{2}{|c|}{ Delta } & \multicolumn{2}{|c|}{ FY 1996} \\
\hline & FTES & $\$ s$ & FTES & $\$ s$ & FTES & $\$ s$ & FTES & $\$ s$ & FTES & $\$ s$ \\
\hline $\begin{array}{l}\text { Work Scope Detailed Descriptions: } \\
\text { continued from previous page- } \\
\text { - Upgrade existing process for tracking, trending, } \\
\text { analyzing and communicating RadCon program } \\
\text { weaknesses, and needed improvement actions, } \\
\text { based upon data generated from Radiological } \\
\text { Problem Reports, the event reporting system, } \\
\text { self assessments and internal reviews, audits, } \\
\text { appraisals and surveillance reports. } \\
\text { - Track and report the RC self assessment process } \\
\text { and integrate improvement actions into fiscal } \\
\text { year work plans and strategic planning process. } \\
\text { - Maintain and distribute lessons learned from } \\
\text { assessments and other information sources. } \\
\text { Coordinate the RadCon Program Tri-Annual } \\
\text { assessment program as required by the HSRCM } \\
\text { Provide detailed assessments and administrative } \\
\text { review of the RWP system and Radiological } \\
\text { Control records. }\end{array}$ & & & & & & & & & & . \\
\hline WHC LABOR COSTS & 4.0 & 260.0 & & & 4.0 & 260.0 & & & 4.0 & 267.8 \\
\hline
\end{tabular}




\begin{tabular}{|c|c|c|c|c|c|c|c|c|c|c|}
\hline \multirow{3}{*}{$\begin{array}{l}\text { Cost Account No. } 1 \mathrm{~J} 330 \mathrm{~A} 0800 \mathrm{~J} 33 \mathrm{AB} \\
\text { Element Task Description } \\
\text { Requests Within Target: }\end{array}$} & \multicolumn{10}{|c|}{ Part II - Management \& Administration - Radiological Control } \\
\hline & \multicolumn{2}{|c|}{1994 FYSF } & \multicolumn{2}{|c|}{ Delta } & \multicolumn{2}{|c|}{ FY 1995} & \multicolumn{2}{|c|}{ Delta } & \multicolumn{2}{|c|}{ FY 1996} \\
\hline & FTES & \$s & FTEs & \$s & FTES & \$s & FTES & $\$ s$ & FTES & $\$ s$ \\
\hline $\begin{array}{l}\text { Work Scope Detailed Descriptions: } \\
\text { Coordinate the development of a continuously } \\
\text { improving set of multi-year and fiscal year } \\
\text { Radiological Protection Program plans, in accordance } \\
\text { with Site Management System guidelines and schedules. } \\
\text { Define and administer the status reporting, } \\
\text { commitment management, and change control processes } \\
\text { associated with implementation of these plans. } \\
\text { - } \quad \text { Provide ongoing support and guidance to the } \\
\text { Radiological Control organization in the } \\
\text { development and maintenance of the Fiscal Year } \\
\text { Work Plan. } \\
\text { Act as the Radiological Control single point of } \\
\text { contact for all activities both internally and } \\
\text { externally to the organization relating to the } \\
\text { Fiscal Year Work Plan. } \\
\text { Oversee the development and submittal of all } \\
\text { program reports including the monthly SMS } \\
\text { report, monthly program review, bi-monthly } \\
\text { program review, and monthly management briefing. } \\
\text { Coordinate all presentations. } \\
\text { Maintain a commitment tracking system and } \\
\text { documentation files on all FYWP milestones and } \\
\text { associated required actions. }\end{array}$ & & & & & & & & & & \\
\hline WHC LABOR COSTS & 1.8 & 117.0 & & & 1.0 & 65.0 & & & 1.0 & 67.0 \\
\hline
\end{tabular}




\begin{tabular}{|c|c|c|c|c|c|c|c|c|c|c|}
\hline \multirow{3}{*}{$\begin{array}{l}\text { Cost Account No. 1J330A0800 J33A8 } \\
\text { Element Task Description } \\
\text { Requests Within Target: }\end{array}$} & \multicolumn{10}{|c|}{ Part II - Management \& Administration - Radiological Control } \\
\hline & \multicolumn{2}{|c|}{1994 FYSF } & \multicolumn{2}{|c|}{ Delta } & \multicolumn{2}{|c|}{ FY 1995} & \multicolumn{2}{|c|}{ Delta } & \multicolumn{2}{|c|}{ FY 1996} \\
\hline & FTES & \$s & FTES & \$s & FTES & \$s & FTES & \$s & FTEs & \$s \\
\hline $\begin{array}{l}\text { Hork Scope Detailed Descriptions: } \\
\text { Define, actively promote, and guide the } \\
\text { implementation of a strategic planning process for } \\
\text { Radiological Control which will serve as an effective } \\
\text { "head-end" input to the SMS functional planning } \\
\text { process. The process must provide a mechanism for } \\
\text { the Radiological Control leadership team and key } \\
\text { stakeholder to regularly reassess the vision, } \\
\text { mission, values, priorities, major issues, and } \\
\text { strategies associated with both the Radiological } \\
\text { Control organization and the WHC RadCon program. } \\
\text { - Coordinate Radiological Control short and long } \\
\text { range strategic planning based on external } \\
\text { assessments, audits, and appraisals and } \\
\text { specifically identified program and facility } \\
\text { needs. } \\
\text { - Actively promote, coordinate, and track } \\
\text { organizational Total Quality Improvement action } \\
\text { and plans. Take a leadership role in the } \\
\text { Radiological Control Total Quality process. }\end{array}$ & & & & & & & & & & \\
\hline WHC LABOR COSTS & 1.0 & 65.0 & & & .2 & 13.0 & & & .2 & 13.4 \\
\hline
\end{tabular}




\begin{tabular}{|c|c|c|c|c|c|c|c|c|c|c|}
\hline Cost Account No. 1J330A0800 J33A8 & \multicolumn{10}{|c|}{ Part II - Management \& Administration - Radiological Control } \\
\hline Element Task Description & 1994 & YSF & De & & FY & & & & FY & \\
\hline Requests Within Target: & FTES & $\$ s$ & FTES & $\$ s$ & FTES & $\$ \mathbf{s}$ & FTES & $\$ \mathbf{s}$ & FTES & $\$ s$ \\
\hline $\begin{array}{l}\text { Work scope Detailed Description: } \\
\text { Upgrade the thoroughness and quality of } \\
\text { administrative processes, plans, and reports produced } \\
\text { by Radiological Control. Promote a more effective } \\
\text { network of administrative resources throughout } \\
\text { Radiological Control. off-load more routine, } \\
\text { administrative responsibilities from the Radiological } \\
\text { Control line managers, and encourage a broader sense } \\
\text { of ownership by and empowerment of Radiological } \\
\text { Control administrative professionals for overall } \\
\text { performance in their specific areas of } \\
\text { responsibility. } \\
\text { - Support Radiological Control field activities by } \\
\text { maintaining the Radiological Control Records } \\
\text { System. Gather documents from the field, scan } \\
\text { as appropriate, and prepare for long term } \\
\text { storage. } \\
\text { - Act as the Peoplecore representative for the } \\
\text { Radiological Control organization. Develop and } \\
\text { maintain a current Radiological Control } \\
\text { directory. } \\
\text { Maintain forms control for the Radiological } \\
\text { Control organization. Ensure appropriate } \\
\text { quantity of forms are maintained on hand. }\end{array}$ & & & & & & & & & & \\
\hline
\end{tabular}




\begin{tabular}{|c|c|c|c|c|c|c|c|c|c|c|}
\hline Cost Account No. 1J330A0800 J33A8 & \multicolumn{10}{|c|}{ Part II - Management \& Administration - Radiological Control } \\
\hline Element Task Description & \multicolumn{2}{|c|}{1994 FYSF } & \multicolumn{2}{|c|}{ Delta } & \multicolumn{2}{|c|}{ FY 1995} & \multicolumn{2}{|c|}{ Delta } & \multicolumn{2}{|c|}{ FY 1996} \\
\hline Requests Within Target: & FTES & \$s & FTES & $\$ \mathbf{s}$ & FTES & \$s & FTES & $\$ s$ & FTES & \$s \\
\hline $\begin{array}{l}\text { Hork Scope Detailed Description: } \\
\text { cont inued from previous page- } \\
\text { Coordinate the Radiological Control and } \\
\text { Radiological Control program Performance } \\
\text { Indicators to be included in appropriate company } \\
\text { measurement tools. } \\
\text { - Provide Great Ideas coordination for the } \\
\text { Radiological Control organization. } \\
\text { - Track and report organizational performance } \\
\text { against the QUEST system and RPR system. } \\
\text { - Coordinate, issue and maintain the WHC release } \\
\text { exempt facilities listing. } \\
\text { - Misc Administrative activities-Provide Single } \\
\text { Point of Contact for all Radiological Control } \\
\text { Union related activities. Coordinate Lead Boards } \\
\text { and aid in the resolution of Union related } \\
\text { issues. } \\
\text { Coordinate the hiring of all new HPTs for the } \\
\text { Radiological Control organization. Provide } \\
\text { management responsibilities for the new HPTs } \\
\text { until assigned to field units. } \\
\text { Provide Employee Concerns coordination for the } \\
\text { Radiological Control organization. }\end{array}$ & & & & & & & & & & \\
\hline WHC Labor Cost & 5.9 & 383.5 & & & 3.9 & 253.5 & & & 3.9 & 261.0 \\
\hline
\end{tabular}




\begin{tabular}{|c|c|c|c|c|c|c|c|c|c|c|}
\hline Cost Account No. 1J330A0800 J33A8 & \multicolumn{10}{|c|}{ Part II - Management \& Administration - Radiological Control } \\
\hline Element Task Description & \multicolumn{2}{|c|}{1994 FYSF } & \multicolumn{2}{|c|}{ Delta } & \multicolumn{2}{|c|}{ FY 1995} & \multicolumn{2}{|c|}{ Delta } & \multicolumn{2}{|c|}{ FY 1996} \\
\hline Requests Within Target: & FTES & $\$$ \$ & FTEs & $\$ s$ & FTEs & $\$ s$ & FTEs & $\$ \mathbf{s}$ & FTEs & $\$ s$ \\
\hline Total Labor & 18.6 & 1209.0 & & & 15.0 & 909.8 & & & 15.0 & 937.1 \\
\hline $\begin{array}{l}\text { (List oNLY items that exceed } \$ 5 k \text {. (i.e., } 20 \text { computers]) } \\
\text { Non-Labor Cost Descriptions }\end{array}$ & & & & & & & & & & \\
\hline $\begin{array}{l}\text { Non-Labor Costs, materials, purchase order } \\
\text { consultants, computers and misc. }\end{array}$ & & & & & & 305.9 & & & & 315.1 \\
\hline Total Non-Labor & & & & & & 305.9 & & & & 315.1 \\
\hline Total Cost Account Within Target & 18.6 & 1209.0 & & & 15.0 & 1215.7 & & & 15.0 & 1252.2 \\
\hline
\end{tabular}




\begin{tabular}{|c|c|c|}
\hline $\begin{array}{c}\text { 3.A Work Breakdown Structure } \\
\text { Dictionary }\end{array}$ & $\begin{array}{l}\text { Westinghouse Hanford Company } \\
\text { Radiological Control }\end{array}$ & $\begin{array}{c}\text { FY } 1995 \\
\text { Site Support Program Plan }\end{array}$ \\
\hline $\begin{array}{l}\text { Cost Account No. } \\
1 J 330 A 0601 \quad J 3360\end{array}$ & Part II - Element Definition & $\begin{array}{c}\text { Date Prepared: August } 30,1994 \\
\text { Revision \#0 }\end{array}$ \\
\hline
\end{tabular}

\begin{tabular}{|l|l|}
\hline SMS WBS code: 6.7 .2 .4 .2 & cost Account title: RADIOLOGICAL ENGINEERING and ALARA \\
\hline
\end{tabular}

Technical Description/Objectives:

Radiological Engineering and ALARA support the implementation of the Radiological Control Program through Technical Radiological Control support. These activities are supported by these work groups; Dosimetry, which includes Radiological ALARA (Dosimetry is addressed in a separate Work Breakdown Structure Dictionary), and the Contamination Control Improvement Project, Radiological Control Technology, and Radiological Engineering.

Radiological Control Technology performs radiological safety activities to assist line management in the implementation of radiological safety requirements, resolve particular radiation safety problems, and provide technical management for centralized radiological control programs.

Objectives:

- To evaluate and develop criteria, work practices, and task procedures that assist line management in implementation of compliance requirements specified by DOE Orders, 10 CFR 835, and Company standards.

- To resolve technical radiological safety problems identified within various Hanford facilities. These problems are outside the scope of general activities and the skill of facility Radiological Control personnel and require a mix of specialty engineer skills on a short-term bases.

- To provide technical management of centralized Radiological Control programs for radioactive source control, radiation generating device control, quality control of analytical instruments for radioactive analysis, coordination of calibration and maintenance services for survey instruments, field implementation of work place air sampling, and performance evaluation of personnel survey devices.

- To represent WHC in technical areas of the Radiation Protection Program when interfacing with DOE, other contractors and regulatory agencies. 


\begin{tabular}{|c|c|c|c|}
\hline 3.A Work & $\begin{array}{l}\text { Breakdown Structure } \\
\text { Dictionary }\end{array}$ & $\begin{array}{l}\text { Westinghouse Hanford Company } \\
\text { Radiological Control }\end{array}$ & $\begin{array}{c}\text { FY } 1995 \\
\text { Site Support Program Plan }\end{array}$ \\
\hline $\begin{array}{l}\text { Cost Account No. } \\
1 \mathrm{~J} 330 \mathrm{~A} 0601\end{array}$ & $J 3360$ & Part II - Element Definition & $\begin{array}{c}\text { Date Prepared: August } 30,1994 \\
\text { Revision \#0 }\end{array}$ \\
\hline
\end{tabular}

\begin{tabular}{|l|l|}
\hline SMS WBS Code: $6.7 \cdot 2 \cdot 4.2$ & cost Account title: RADIOLOGICAL ENGINEERING and ALARA \\
\hline
\end{tabular}

Radiological Control Technical Services develops and maintain work practices and procedure, exercise oversight of the radiological safety training program, administer the Hanford Safety Bulletin Board, and provide various Radiological Control Engineering Support Activities.

Objectives:

- To produce quality and field usable work practices and procedures.

- To ensure that procedures and work practices meet the applicable regulatory requirements.

- To enhance training effectiveness and communications throughout Radiological Control related programs.

Milestones: (See Section 3.8 Milestone Description Sheets for details)

None 


\begin{tabular}{|c|c|c|c|c|c|c|c|c|c|c|}
\hline \multirow{3}{*}{$\begin{array}{l}\text { Cost Account No. 1J330A0601 J3360 } \\
\text { Element Task Description } \\
\text { Requests Within Target: }\end{array}$} & \multicolumn{10}{|c|}{ Part II - Radiological Engineering and ALARA } \\
\hline & \multicolumn{2}{|c|}{1994 FYSF } & \multicolumn{2}{|c|}{ Delta } & \multicolumn{2}{|c|}{ FY 1995} & \multicolumn{2}{|c|}{ Delta } & \multicolumn{2}{|c|}{ FY 1996} \\
\hline & FTES & \$s & FTES & \$s & FTEs & $\$ s$ & FTEs & \$s & FTES & \$s \\
\hline $\begin{array}{l}\text { Hork Scope Detailed Descriptions: } \\
\text { Radiological Engineering and ALARA Management } \\
\text { function provides management oversight and direction } \\
\text { for the various functions. This includes technical, } \\
\text { administrative, and clerical support. } \\
\text { Provide coordination of HPTs activities in support } \\
\text { of the compliant implementation of the Radiological } \\
\text { Control Program. Activities are planned and } \\
\text { scheduled to meet organizational and program } \\
\text { priorities effectively within assigned time frames. } \\
\text { Decreases reflect organizational management } \\
\text { structure streamlining. }\end{array}$ & & & & & & & & & & \\
\hline WHC Labor Costs & 5.0 & 325.0 & & & 4.0 & 260.0 & & & 4.0 & 267.8 \\
\hline $\begin{array}{l}\text { Hork Scope Detailed Descriptions: } \\
\text { Radiological Work Practices/Procedures Development } \\
\text { Develop and maintain Radiological Control Practices } \\
\text { and Procedures. Perform periodic review, preserve } \\
\text { historical records on processes, and initiate } \\
\text { effective change control through document change } \\
\text { requests. Interface with responsible organizations } \\
\text { for instructional support and implementation } \\
\text { strategies. }\end{array}$ & & & & & & & & & & ' \\
\hline WHC LABOR COSTS & 5.0 & 325.0 & & & 5.0 & 325.0 & & & 5.0 & 334.6 \\
\hline
\end{tabular}




\begin{tabular}{|c|c|c|c|c|c|c|c|c|c|c|}
\hline Cost Account No. $1 \mathrm{~J} 330 A 0601 \mathrm{~J} 3360$ & \multicolumn{10}{|c|}{ Part II - Radiological Engineering and ALARA } \\
\hline Element Task Description & \multicolumn{2}{|c|}{1994 FYSF } & \multicolumn{2}{|c|}{ Delta } & \multicolumn{2}{|c|}{ FY 1995} & \multicolumn{2}{|c|}{ Delta } & \multicolumn{2}{|c|}{ FY 1996} \\
\hline Requests Within Target: & FTEs & \$s & FTES & $\$ \mathbf{s}$ & FTEs & $\$ s$ & FTES & $\$ \mathbf{s}$ & FTEs & \$s \\
\hline $\begin{array}{l}\text { Work Scope Detailed Descriptions: } \\
\text { Radiological safety training oversight } \\
\text { Provide review and concurrence of existing and } \\
\text { future radiological safety training to include: } \\
\text { Visitor orientation, Facility orientation, HGET, Ra } \\
\text { Worker I, Rad Worker II, Health Physics Technician } \\
\text { (Trainee), Health Physics Technician (certificatio } \\
\text { re-certification), Radiological Control Managers, } \\
\text { and Analysts. Radiological Control Facility/Generi } \\
\text { OJT program. }\end{array}$ & & & & & & & & & & \\
\hline WHC LABOR COSTS & 1.0 & 65.0 & & & 1.0 & 65.0 & & & 1.0 & 67.0 \\
\hline
\end{tabular}




\begin{tabular}{|c|c|c|c|c|c|c|c|c|c|c|}
\hline \multirow{3}{*}{$\begin{array}{l}\text { Cost Account No. 1J330A0601 J3360 } \\
\text { Element Task Description } \\
\text { Requests Within Target: }\end{array}$} & \multicolumn{10}{|c|}{ Part II - Radiological Engineering and ALARA } \\
\hline & \multicolumn{2}{|c|}{1994 FYSF } & \multicolumn{2}{|c|}{ Delta } & \multicolumn{2}{|c|}{ FY 1995} & \multicolumn{2}{|c|}{ Delta } & \multicolumn{2}{|c|}{ FY 1996} \\
\hline & FTEs & $\$ s$ & FTEs & $\$ s$ & FTEs & $\$ 5$ & FTEs & $\$ s$ & FTEs & $\$ s$ \\
\hline $\begin{array}{l}\text { Work Scope Detailed Descriptions: } \\
\text { Radiological Control Technology Management provides } \\
\text { coordination of Radiological Control Technology } \\
\text { activities in support of the compliant } \\
\text { implementation of the Radiological Control Program } \\
\text { as required by DOE/EH-0256T, Articles } 141.1 \text { and } 143 \text {, } \\
\text { DOE Orders } 5400.5,5480.11,5480.19,5480.20 \text {, and } \\
10 \text { CFR } 835 \text {. Activities are planned and scheduled to } \\
\text { meet organizational and program priorities } \\
\text { effectively within assigned timeframes. Technical } \\
\text { and administrative management is provided for } \\
\text { specialty radiological safety engineers and site } \\
\text { cognizant engineer programs of Radiological Control. } \\
\text { Decreases reflect organizational management } \\
\text { structure streamlining. }\end{array}$ & & & & & & & & & & \\
\hline WHC Labor Costs & 2.0 & 92.0 & & & 2.0 & 92.0 & & & 2.0 & 95.8 \\
\hline Total Labor for Cost Account & 13.0 & 807.0 & & & 12.0 & 741.3 & & & 12.0 & 770.9 \\
\hline $\begin{array}{l}\text { Non-Labor items that exceed } \$ 5 K \text {. [i.e., } 20 \text { Computers]) } \\
\text { Non-Labor Cost Descriptions }\end{array}$ & & & & & & & & & & \\
\hline NON-Labor, materials and misc. costs. & & 659.0 & & & & 209.0 & & & & 217.4 \\
\hline P. 0 Contracts & & & & & & 450.0 & & & & 468.0 \\
\hline Total Non-Labor & & & & & & 659.0 & & & & 685.4 \\
\hline Total Cost Account Within Target & 13.0 & 1466.0 & & & 12.0 & 1400.3 & & 56.0 & 12.0 & 1456.3 \\
\hline
\end{tabular}




\begin{tabular}{|c|c|c|}
\hline $\begin{array}{l}\text { 3.A Work Breakdown Structure } \\
\text { Dictionary }\end{array}$ & \multirow{2}{*}{$\begin{array}{l}\text { West inghouse Hanford Company } \\
\text { Radiological Control } \\
\text { Part II - Element Definition }\end{array}$} & \multirow{2}{*}{$\begin{array}{c}\text { Fy } 1995 \\
\text { Site Support Program Plan } \\
\text { Date Prepared: August } 30,1994 \\
\text { Revision \#0 }\end{array}$} \\
\hline $\begin{array}{l}\text { Cost Account No. } \\
1 \mathrm{J330A0100} \quad \mathrm{J3301}\end{array}$ & & \\
\hline SMS WBS code: 6.7 .4 .4 .1 & \multicolumn{2}{|c|}{ Cost Account Title: Southern Areas and Support Services Radiological Control } \\
\hline \multicolumn{3}{|c|}{$\begin{array}{l}\text { Provide management and administrative support to assure safe, effective conduct of operations for SA\&SS. Provide and } \\
\text { help implement an effective and efficient radiological safety program for } 300 \text { and } 400 \text { Areas facility operations, } \\
\text { landlord operations, fleet maintenance, site materials management, laundry operations, and project management } \\
\text { activities. Provide radiological sampling and surveying support for the "active site" environmental assurance program } \\
\text { and well maintenance. Provide prompt radiological safety response capability for off-normal and emergency conditions } \\
\text { for the RL Radiological Assistance Program. Provide training, financial, and materials management services together } \\
\text { with the implementation of consolidated radiological policy and procedure reviews. } \\
\text { milestones: (See section 3.8 milestone Description sheets for details) }\end{array}$} \\
\hline
\end{tabular}




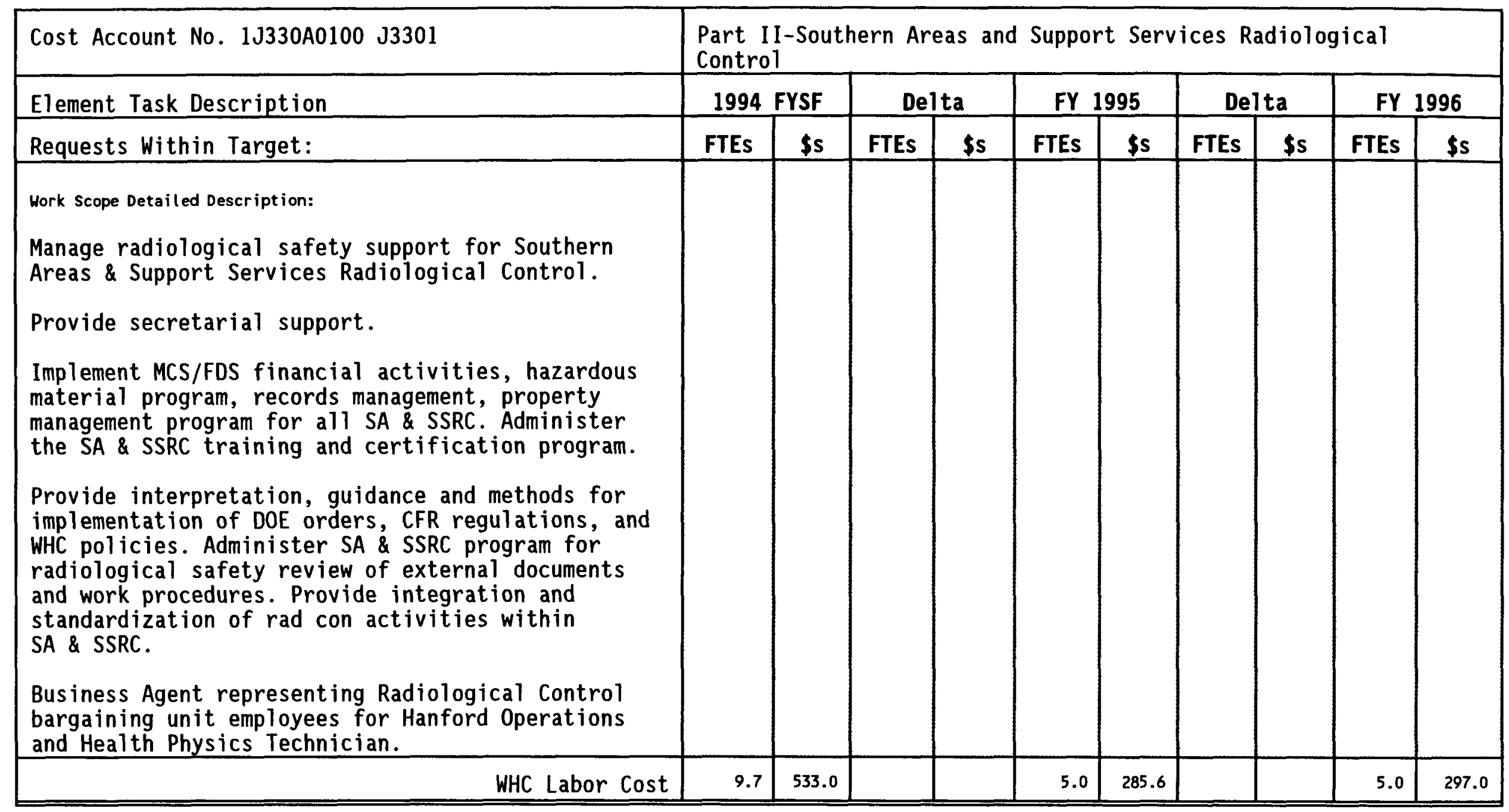




\begin{tabular}{|c|c|c|c|c|c|c|c|c|c|c|}
\hline \multirow{3}{*}{\begin{tabular}{|l} 
Cost Account No. $1 \mathrm{~J} 330 \mathrm{A0} 100 \mathrm{~J} 3301$ \\
Element Task Description \\
Requests Within Target: \\
\end{tabular}} & \multicolumn{10}{|c|}{$\begin{array}{l}\text { Part II-Southern Areas and Support Services Radiological } \\
\text { Control }\end{array}$} \\
\hline & \multicolumn{2}{|c|}{1994 FYSF } & \multicolumn{2}{|c|}{ Delta } & \multicolumn{2}{|c|}{ FY 1995} & \multicolumn{2}{|c|}{ Delta } & \multicolumn{2}{|c|}{ FY 1996} \\
\hline & FTES & $\$ s$ & FTES & \$s & FTES & $\$ s$ & FTES & $\$ s$ & FTES & $\$ s$ \\
\hline \multicolumn{11}{|l|}{$\begin{array}{l}\text { Work scope Detailed Description: } \\
\text { Radiological Control provide a supplemental team of } \\
5 \text { HPTs which are used as needed around the site. } \\
\text { The labor of thes technicians is charged to the } \\
\text { projects on a work performed basis. }\end{array}$} \\
\hline 1 & & & & & & & & & & \\
\hline WHC Labor Cost & 5.0 & 287.6 & & & 5.0 & & & & 5.0 & \\
\hline
\end{tabular}

1 These HPTs are funded by direct charges to various programs. Radiological Control assumes their training and overhead costs. 


\begin{tabular}{|c|c|c|c|c|c|c|c|c|c|c|}
\hline \multirow{3}{*}{$\begin{array}{l}\text { Cost Account No. 1J330A0100 J3301 } \\
\text { Element Task Description } \\
\text { Requests Within Target: }\end{array}$} & \multicolumn{10}{|c|}{$\begin{array}{l}\text { Part II-Southern Areas and Support Services Radiological } \\
\text { Control }\end{array}$} \\
\hline & \multicolumn{2}{|c|}{1994 FYSF } & \multicolumn{2}{|c|}{ Delta } & \multicolumn{2}{|c|}{ FY 1995} & \multicolumn{2}{|c|}{ Delta } & \multicolumn{2}{|c|}{ FY 1996} \\
\hline & FTES & \$s & FTES & $\$ s$ & FTES & $\$ s$ & FTES & $\$ s$ & FTES & $\$ s$ \\
\hline $\begin{array}{l}\text { Work Scope Detailed Description: } \\
\text { Southern Areas and Support Services Radiological } \\
\text { Control provides services for routine survey, soil, } \\
\text { air, and water samples and small operation facility } \\
\text { radiological control programs which include a large } \\
\text { part of the Hanford site. A large amount of this } \\
\text { work is performed in the } 300 \text { Area for a number of } \\
\text { customers. Radiological Control provides } \\
\text { administration and training for } 38 \text { HPTs employed in } \\
\text { this function. } 2\end{array}$ & & & & & & & & & & \\
\hline WHC Labor Cost & 38.0 & & & & 38.0 & & & & 38.0 & \\
\hline Total Labor & & & & & 5.0 & 285.6 & & & 5.0 & 297.0 \\
\hline (List ONLY items that exceed \$5K. [i.e., 20 Computers]) & & & & & & & & & & \\
\hline $\begin{array}{l}\text { Non-Labor Cost Descriptions } \\
\text { Non-Labor cost include materials, purchased } \\
\text { services, training and IRM support. }\end{array}$ & & & & & & & & & & \\
\hline Total Non-Labor & & 70.5 & & & & 99.1 & & & & 103.1 \\
\hline Total Cost Account Within Target & 9.7 & 645.1 & & & 5.0 & 384.7 & & 15.4 & 5.0 & 400.1 \\
\hline
\end{tabular}

2 These HPTs are funded by direct charges to various programs. Radiological Control assumes their training and overhead costs. 


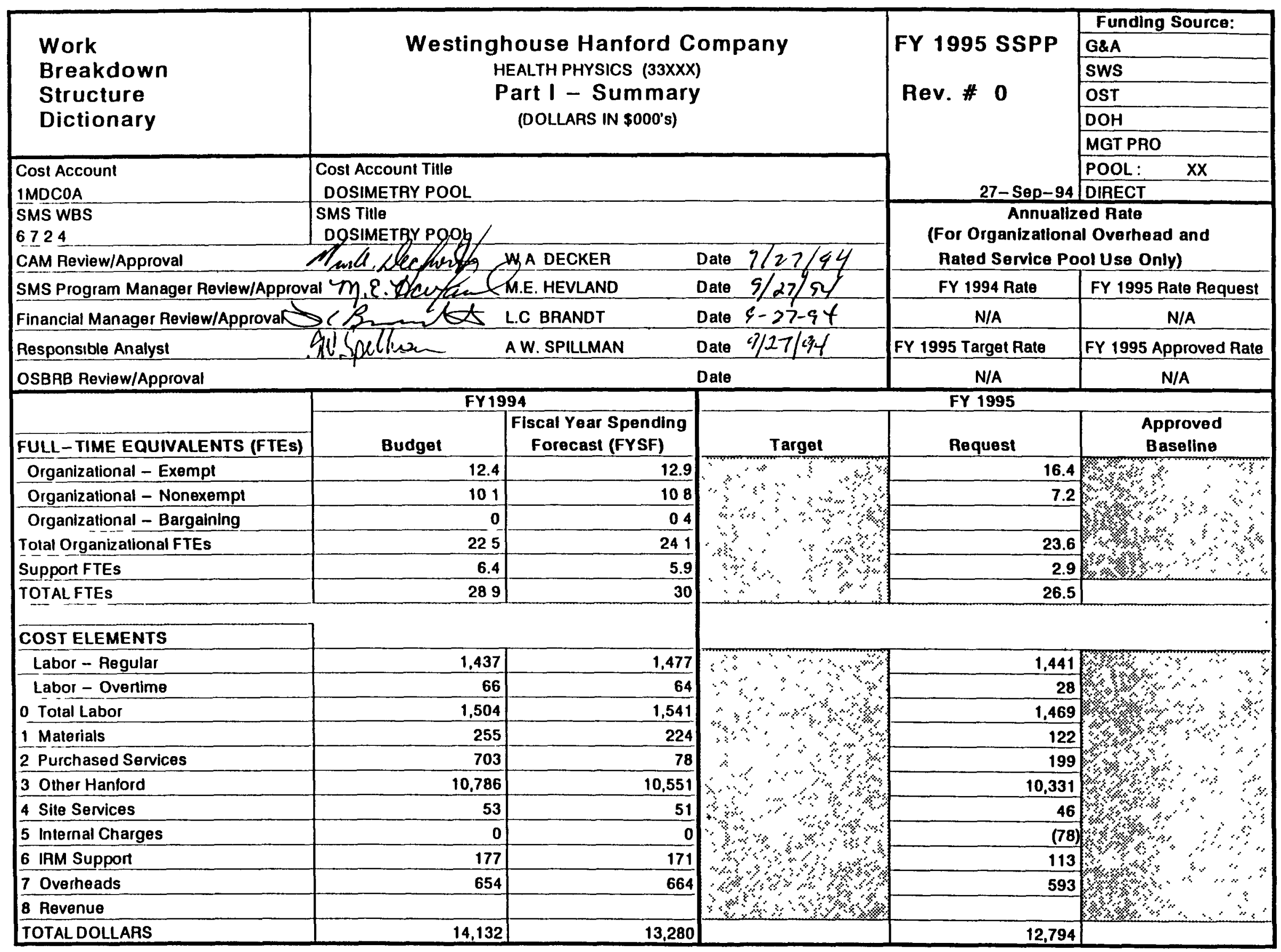

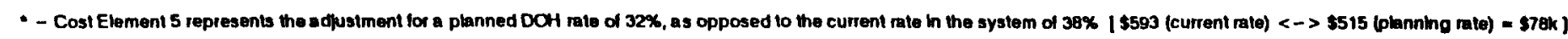




\begin{tabular}{|c|c|c|}
\hline $\begin{array}{c}\text { 3. A Work Breakdown Structure } \\
\text { Dictionary }\end{array}$ & \multirow{2}{*}{$\begin{array}{l}\text { Westinghouse Hanford Company } \\
\text { Radiological Control } \\
\text { Part II - Element Definition }\end{array}$} & \multirow{2}{*}{$\begin{array}{c}\text { FY } 1995 \\
\text { Site Support Program Plan } \\
\text { Date Prepared: August 30, } 1994 \\
\text { Revision \#0 }\end{array}$} \\
\hline $\begin{array}{l}\text { Cost Account No. } \\
\text { IMDCOA }\end{array}$ & & \\
\hline SMS WBS Code: $\quad 6.7 .2 \cdot 4 \cdot 2.1$ & \multicolumn{2}{|l|}{ Cost Account Title: Dosimetry } \\
\hline \multicolumn{3}{|c|}{$\begin{array}{l}\text { Administers the Westinghouse Hanford Company dosimetry program for BCSR, ICF KH and WHC employees and their } \\
\text { subcontractors; DOE-RL; and visitors. }\end{array}$} \\
\hline \multicolumn{3}{|c|}{$\begin{array}{l}\text { Administers the WHC Instrumentation and Calibration Services for portable instrumentation. } \\
\text { Radiological ALARA Program and the Contamination Control Improvement Project. }\end{array}$} \\
\hline
\end{tabular}




\begin{tabular}{|c|c|c|}
\hline $\begin{array}{c}\text { 3. A Work Breakdown Structure } \\
\text { Dictionary }\end{array}$ & $\begin{array}{c}\text { Westinghouse Hanford Company } \\
\text { Radiological Control }\end{array}$ & $\begin{array}{c}\text { FY } 1995 \\
\text { Site Support Program Plan }\end{array}$ \\
\hline $\begin{array}{l}\text { Cost Account No. } \\
\text { IMDCOA }\end{array}$ & Part II - Element Definition & $\begin{array}{c}\text { Date Prepared: August } 30,1994 \\
\text { Revision \#0 }\end{array}$ \\
\hline
\end{tabular}

- Manage a dosimetry program which meets all regulatory requirements and which provides effective and technically astute support to field operations.

- Coordinate calibration of WHC portable radiation instrumentation with PNL Calibration and Instrumentation Services.

- Administer the dosimetry program to assure accurate and timely recording and reporting of radiation exposure data.

- Coordinate activities of the Radiological Control organization which impact WHC ALARA Program Office objectives.

- Monitor and assess WHC dosimetry experience and report exposure performance.

- Manage a radiological area access control program which meets Facility and DoE requirements and optimizes the production process.

- Improve the chain of custody process for routine dosimeter issue and retrieval with end users.

- Provide support for the workplace air sampling and respiratory protection programs

- Provide support to the WHC Radiological ALARA Program.

- Support the WHC Contamination Control Improvement Project. 


\begin{tabular}{|c|c|c|}
\hline $\begin{array}{l}\text { 3.A Work Breakdown Structure } \\
\text { Dictionary }\end{array}$ & $\begin{array}{l}\text { Westinghouse Hanford Company } \\
\text { Radiological Control }\end{array}$ & $\begin{array}{l}\text { FY } 1995 \\
\text { Site Support Program Plan }\end{array}$ \\
\hline $\begin{array}{l}\text { Cost Account No. } \\
\text { 1MDCOA }\end{array}$ & Part II - Element Definition & $\begin{array}{c}\text { Date Prepared: August 30, } 1994 \\
\text { Revision \#0 }\end{array}$ \\
\hline
\end{tabular}

\begin{tabular}{|l|l|}
\hline SMS WBS Code: $6 \cdot 7 \cdot 2 \cdot 4 \cdot 2.1$ & Cost Account ritle: Dosimetry \\
\hline
\end{tabular}

Milestones: (See Section 3.B Milestone Description Sheets for details)

- RAD-95-010 Perform ALARA Program Review with DOE-RL

$12 / 30 / 94$

- RAD-95-011 Perform ALARA Program Review with DOE-RL

$03 / 31 / 95$

- RAD-95-012 Perform ALARA Program Review with DOE-RL

$06 / 30 / 95$

- RAD-95-013 Perform ALARA Program Review with DOE-RL

$09 / 30 / 95$

- RAD-95-014 Report of WHC FY 1995 ALARA Performance Goals

$11 / 15 / 94$

- RAD-95-015 Summary Report for CY 1994 ALARA Goals

$04 / 28 / 95$

- RAD-95-020 Reduce No. of people in external dosimetry Program

$09 / 30 / 95$

- RAD-95-021 Reduce No. of people in internal dosimetry Program 


\begin{tabular}{|c|c|c|c|c|c|c|c|c|c|c|}
\hline Cost Account No. IMDCOAM200 MDPNL & \multicolumn{10}{|c|}{$\begin{array}{l}\text { Part II - Radiological Control--Dosimetry-Portable Instrument } \\
\text { Calibration- }\end{array}$} \\
\hline Element Task Description & \multicolumn{2}{|c|}{1994 FYSF } & \multicolumn{2}{|c|}{ Delta } & \multicolumn{2}{|c|}{ FY 1995} & \multicolumn{2}{|c|}{ Delta } & \multicolumn{2}{|c|}{ FY 1996} \\
\hline Requests Within Target: & FTEs & $\$ s$ & FTES & $\$ \mathbf{s}$ & FTES & $\$$ s & FTES & $\$ s$ & FTES & $\$ s$ \\
\hline $\begin{array}{l}\text { Hork Scope Detailed Description: } \\
\text { Portable Instrumentation and Calibration } \\
\text { Coordinate all WHC activities with the PNL } \\
\text { Instrument and Calibration Services relating to the } \\
\text { calibration and maintenance of survey instruments } \\
\text { and electronic personnel dosimetry as required by } \\
\text { DOE RCM } 513 \text {, } 551 \text {, } 562,563 \text { and } 564 \text {. This task } \\
\text { serves as the single point of contact between WHC } \\
\text { and PNL to coordinate these activities which are } \\
\text { assigned to PNL by the Hanford Site Services } \\
\text { Manual. Calibration and maintenance were provided } \\
\text { for more than } 3000 \text { portable radiation survey } \\
\text { instruments and detectors in } 1994 \text {. DOE } \\
\text { Implementation Guide G-10 CFR } 835 / C 2 \text {, G- } \\
\text { 10 CFR } 835 / E 1 \text { and G-10 CFR } 835 / H 1 \text { drive } \\
\text { improvements in this service. }\end{array}$ & & & & & & & & & & \\
\hline WHC Labor Cost & 1.0 & 65.4 & & & 1.0 & 65.2 & & & 1.0 & 68.1 \\
\hline $\begin{array}{l}\text { (List oNLY items that exceed \$5K. [i.e., } 20 \text { Computers]) } \\
\text { Non-Labor Cost Descriptions }\end{array}$ & & & & & & & & & & \\
\hline $\begin{array}{l}\text { Non-Labor cost of portable instrumentation and } \\
\text { calibration paid to PNL }\end{array}$ & & 3486.8 & & & & 3043.8 & & & & 3135.1 \\
\hline Total Non-Labor & & 3486.8 & & & & 3043.8 & & & & 3135.1 \\
\hline Total Cost Account Within Target & 1.0 & 3552.2 & & & 1.0 & 3109.0 & & & 1.0 & 3203.2 \\
\hline
\end{tabular}




\begin{tabular}{|c|c|c|c|c|c|c|c|c|c|c|}
\hline Cost Account No. 1MDCOAM10T M111T & \multicolumn{10}{|c|}{ Part II - Radiological Control--Dosimetry--PNL Oversight- } \\
\hline Element Task Description & \multicolumn{2}{|c|}{1994 FYSF } & \multicolumn{2}{|c|}{ Delta } & \multicolumn{2}{|c|}{ FY 1995} & \multicolumn{2}{|c|}{ Delta } & \multicolumn{2}{|c|}{ FY 1996} \\
\hline Requests Within Target: & FTES & \$s & FTES & $\$ \mathbf{s}$ & FTES & \$s & FTES & \$s & FTES & \$s \\
\hline Total Labor & & & & & & & & & & \\
\hline $\begin{array}{l}\text { (List oNLY items that exceed \$5K. (i.e., } 20 \text { Computers]) } \\
\text { Non-Labor Cost Descriptions }\end{array}$ & & & & & & & & & & \\
\hline PNL Assigned blind audit activities & & & & & & 12.0 & & & & 12.4 \\
\hline Total Non-Labor & & & & & & 12.0 & & & & 12.4 \\
\hline Total Cost Account Within Target & & 12.0 & & & & 12.0 & & & & 12.4 \\
\hline
\end{tabular}

\begin{tabular}{|c|c|c|c|c|c|c|c|c|c|c|}
\hline Cost Account No. IMDCOAM1OW M111W & \multicolumn{10}{|c|}{ Part II - Radiological Control--Dosimetry--PNL Oversight- } \\
\hline Element Task Description & \multicolumn{2}{|c|}{1994 FYSF } & \multicolumn{2}{|c|}{ Delta } & \multicolumn{2}{|c|}{ FY 1995} & \multicolumn{2}{|c|}{ Delta } & \multicolumn{2}{|c|}{ FY 1996} \\
\hline Requests Within Target: & FTES & $\$ \mathbf{s}$ & FTES & \$s & FTEs & $\$ s$ & FTEs & $\$$ s & FTES & $\$ \mathbf{s}$ \\
\hline Total Labor & & & & & & & & & & \\
\hline $\begin{array}{l}\text { (List oNLY items that exceed } 55 \mathrm{~K} \text {. [i.e., } 20 \text { Computers]) } \\
\text { Non-Labor Cost Descriptions }\end{array}$ & & & & & & & & & & \\
\hline PNL Assigned Bioassay Activities & & & & & & 1500.0 & & & & 1545.0 \\
\hline Total Non-Labor & & & & & & 1500.0 & & & & 1545.0 \\
\hline Total cost Account Within Target & & 1800.0 & & & & 1500.0 & & & & 1545.0 \\
\hline
\end{tabular}




\begin{tabular}{|c|c|c|c|c|c|c|c|c|c|c|}
\hline Cost Account No. 1MDCOAM10X M111X & \multicolumn{10}{|c|}{ Part II - Radiological Control--Dosimetry--PNL Oversight- } \\
\hline Element Task Description & \multicolumn{2}{|c|}{1994 FYSF } & \multicolumn{2}{|c|}{ Delta } & \multicolumn{2}{|c|}{ FY 1995} & \multicolumn{2}{|c|}{ Delta } & \multicolumn{2}{|c|}{ FY 1996} \\
\hline Requests Within Target: & FTES & $\$ s$ & FTEs & $\$ \mathbf{s}$ & FTEs & $\$ \mathbf{s}$ & FTEs & $\$ \mathbf{s}$ & FTEs & $\$ \mathbf{s}$ \\
\hline Total Labor & & & & & & & & & & \\
\hline $\begin{array}{l}\text { (List ONLY items that exceed } \$ 5 K \text {. [i.e., } 20 \text { Computers]) } \\
\text { Non-Labor Cost Descriptions }\end{array}$ & & & & & & & & & & \\
\hline PNL Assigned External Dosimetry Activities & & & & & & 1898.0 & & & & 1954.9 \\
\hline Total Non-Labor & & & & & & 1898.0 & & & & 1954.9 \\
\hline Total Cost Account Within Target & & 1993.6 & & & & 1898.0 & & & & 1954.9 \\
\hline
\end{tabular}

\begin{tabular}{|c|c|c|c|c|c|c|c|c|c|c|}
\hline Cost Account No. IMDCOAM1OV M111V & \multicolumn{10}{|c|}{ Part II - Radiological Control--Dosimetry--PNL Oversight- } \\
\hline Element Task Description & \multicolumn{2}{|c|}{1994 FYSF } & \multicolumn{2}{|c|}{ Delta } & \multicolumn{2}{|c|}{ FY 1995} & \multicolumn{2}{|c|}{ Delta } & \multicolumn{2}{|c|}{ FY 1996} \\
\hline Requests Within Target: & FTES & $\$ s$ & FTEs & $\$ s$ & FTES & $\$ s$ & FTEs & $\$ \mathbf{s}$ & FTEs & $\$ s$ \\
\hline Total Labor & & & & & & & & & & \\
\hline $\begin{array}{l}\text { (List oNLY items that exceed \$5K. [i.e., } 20 \text { Computers]) } \\
\text { Non-Labor Cost Descriptions }\end{array}$ & & & & & & & & & & \\
\hline PNL Assigned Site Support Activities & & & & & & 3417.0 & & & & 3519.5 \\
\hline Total Non-Labor & & & & & & 3417.0 & & & & 3519.5 \\
\hline Total Cost Account Within Target & & 3705.7 & & & & 3417.0 & & & & 3519.5 \\
\hline
\end{tabular}




\begin{tabular}{|c|c|c|c|c|c|c|c|c|c|c|}
\hline Cost Account No. IMDCOAM1OY M111Y & \multicolumn{10}{|c|}{ Part II - Radiological Contro1--Dosimetry--PNL Oversight- } \\
\hline Element Task Description & \multicolumn{2}{|c|}{1994 FYSF } & \multicolumn{2}{|c|}{ Delta } & \multicolumn{2}{|c|}{ FY 1995} & \multicolumn{2}{|c|}{ Delta } & \multicolumn{2}{|c|}{ FY 1996} \\
\hline Requests Within Target: & FTES & $\$ \mathbf{S}$ & FTES & $\$ s$ & FTES & $\$ \mathbf{s}$ & FTES & $\$ \mathbf{s}$ & FTES & $\$ s$ \\
\hline Total Labor & & & & & & & & & & \\
\hline $\begin{array}{l}\text { (List ONLY items that exceed } \$ 5 k \text {. [i.e., } 20 \text { Computers]) } \\
\text { Non-Labor Cost Descriptions }\end{array}$ & & & & & & & & & & \\
\hline PNL Assigned Analytical Support Activities & & & & & & 127.5 & & & & 131.3 \\
\hline Total Non-Labor & & & & & & 127.5 & & & & 131.3 \\
\hline Total Cost Account Within Target & & 150.0 & & & & 127.5 & & & & 131.3 \\
\hline
\end{tabular}




\begin{tabular}{|c|c|c|c|c|c|c|c|c|c|c|}
\hline \multirow{3}{*}{$\begin{array}{l}\text { Cost Account No. IMDCOAM103 M111C } \\
\text { Element Task Description } \\
\text { Requests Within Target: }\end{array}$} & \multicolumn{10}{|c|}{$\begin{array}{l}\text { Part II - Radiological Control--Dosimetry WPAS \& Resp } \\
\text { Protection - }\end{array}$} \\
\hline & \multicolumn{2}{|c|}{1994 FYSF } & \multicolumn{2}{|c|}{ Delta } & \multicolumn{2}{|c|}{ FY 1995} & \multicolumn{2}{|c|}{ Delta } & \multicolumn{2}{|c|}{ FY 1996} \\
\hline & FTES & $\$ \mathbf{s}$ & FTES & $\$ s$ & FTES & $\$$ s & FTES & $\$ s$ & FTES & $\$$ s \\
\hline $\begin{array}{l}\text { Work Scope Detailed Description: } \\
\text { Workplace Air Sampling and Respiratory Protection } \\
\text { Programs: } \\
\text { Provide support to field activities in the } \\
\text { implementation and maintenance of the workplace air } \\
\text { sampling program (250 air sampler heads) } \\
\text { (10 CFR } 835.401 \text { ). Support manual updating of } \\
\text { supporting documentation and revisions to facility } \\
\text { program as appropriate. Coordinate with Safety and } \\
\text { Health Technical Services to develop and implement } \\
\text { respiratory protect ion program (DOE RCM 531) } \\
\text { consisting of } 3000 \text { respirators. }\end{array}$ & & & & & & & & & & \\
\hline WHC Labor Cost & 1.0 & 65.4 & & & .3 & 15.0 & & & .3 & 15.5 \\
\hline Total Labor & & & & & & & & & & \\
\hline $\begin{array}{l}\text { (List oNLY items that exceed } \$ 5 K \text {. [i.e., } 20 \text { Computers]) } \\
\text { Non-Labor Cost Descriptions }\end{array}$ & & & & & & & & & & \\
\hline $\begin{array}{l}\text { Non Labor Costs of the work place air sampling } \\
\text { program }\end{array}$ & & & & & & 18.3 & & & & 18.8 \\
\hline Total Non-Labor & & & & & & 18.3 & & & & 18.8 \\
\hline Total Cost Account Within Target & 1.0 & 65.4 & & & .3 & 33.3 & & & .3 & 34.3 \\
\hline
\end{tabular}




\begin{tabular}{|c|c|c|c|c|c|c|c|c|c|c|}
\hline \multirow{3}{*}{$\begin{array}{l}\text { Cost Account No. IMDOAM104 MI11D } \\
\text { Element Task Description } \\
\text { Requests Within Target: } \\
\end{array}$} & \multicolumn{10}{|c|}{ Part II - Radiological Control--Dosimetry-ACES - } \\
\hline & \multicolumn{2}{|c|}{1994 FYSF } & \multicolumn{2}{|c|}{ Delta } & \multicolumn{2}{|c|}{ FY 1995} & \multicolumn{2}{|c|}{ Delta } & \multicolumn{2}{|c|}{ FY 1996} \\
\hline & FTES & $\$ s$ & FTES & \$s & FTES & $\$ s$ & FTEs & $\$ s$ & FTEs & \$s \\
\hline $\begin{array}{l}\text { Work Scope Detailed Description: } \\
\text { Access Control Entry System (ACES) } \\
\text { ACES responds to } 156,000 \text { access requests annually } \\
\text { and provides the management tool (10 CFR } 835.501 a \text { ) } \\
\text { to assure personnel entering radiation areas are } \\
\text { adequately trained, have met the required health } \\
\text { and safety monitoring requirements, and have a } \\
\text { working knowledge of job site conditions relative } \\
\text { to personnel exposure. ACES provides data for } \\
\text { management tracking of personnel radiation exposure } \\
\text { verses task and task location. This is a tool to } \\
\text { establish a sitewide ALARA baseline in support of } \\
\text { pre- and post-job evaluations, ALARA preplanning } \\
\text { and trend analyses (DOE RCM 312, 551). }\end{array}$ & & & & & & & & & & \\
\hline WHC Labor Cost & 3.8 & 249.9 & & & 3.0 & 201.7 & & & 3.0 & 207.7 \\
\hline Total Labor & & & & & & & & & & \\
\hline $\begin{array}{l}\text { (List ONLY items that exceed } 55 K \text {. [i.e., } 20 \text { Computers]) } \\
\text { Non-Labor Cost Descriptions }\end{array}$ & & & & & & & & & & \\
\hline ACES non-labor costs & & & & & & 162.8 & & & & 167.7 \\
\hline Total Non-Labor & & & & & & 162.8 & & & & 167.7 \\
\hline Total Cost Account Within Target & 3.8 & 249.9 & & & 3.0 & 364.5 & & & 3.0 & 375.4 \\
\hline
\end{tabular}




\begin{tabular}{|c|c|c|c|c|c|c|c|c|c|c|}
\hline Cost Account No. IMDCOAM103 M111E & \multicolumn{10}{|c|}{ Part II -Radiological Control--Dosimetry Management - } \\
\hline Element Task Description & \multicolumn{2}{|c|}{1994 FYSF } & \multicolumn{2}{|c|}{ Delta } & \multicolumn{2}{|c|}{ FY 1995} & \multicolumn{2}{|c|}{ Delta } & \multicolumn{2}{|c|}{ FY 1996} \\
\hline Requests Within Target: & FTES & $\$$ s & FTES & \$s & FTES & \$s & FTES & \$s & FTES & \$s \\
\hline 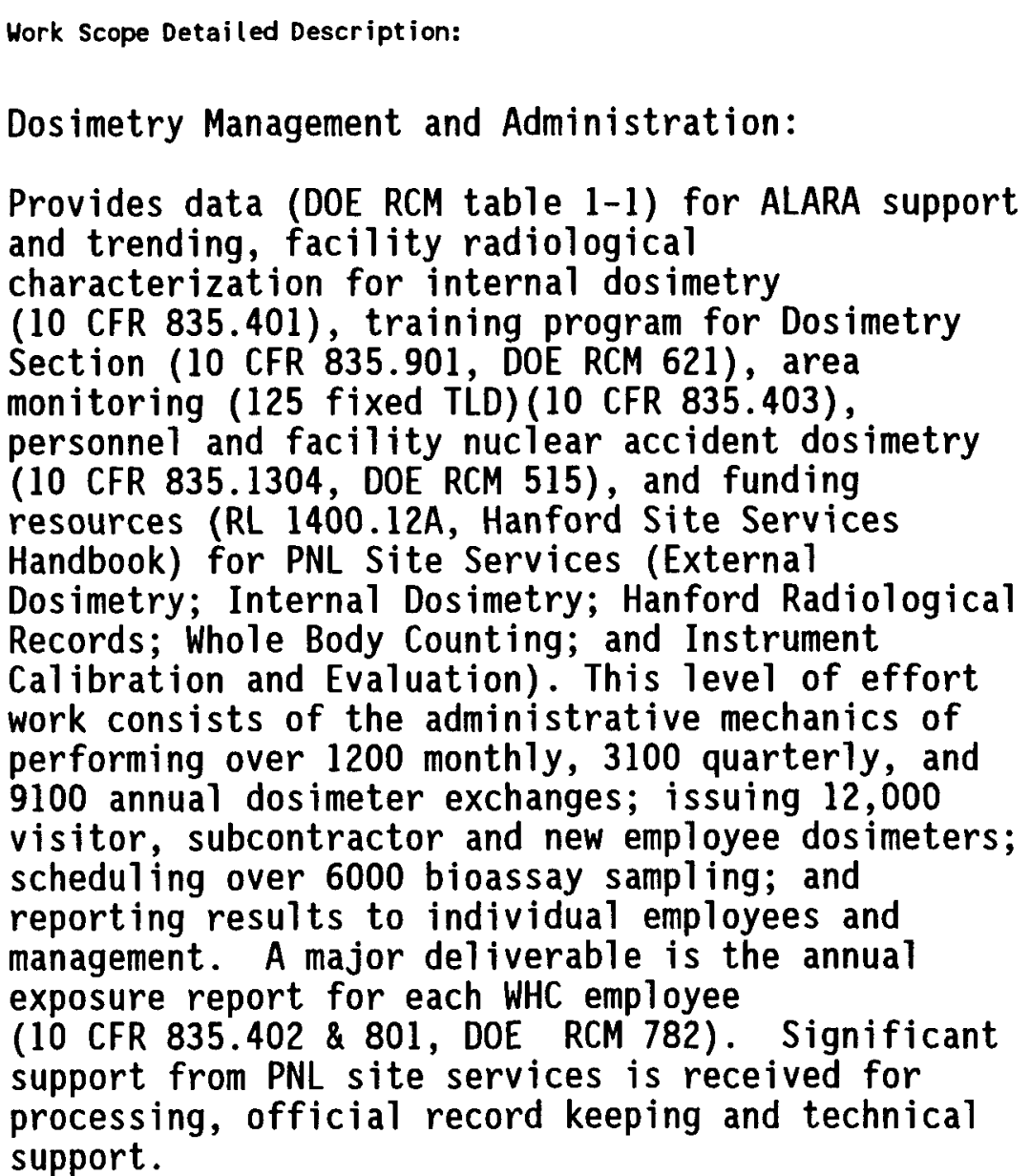 & & & & & & & & & & \\
\hline
\end{tabular}




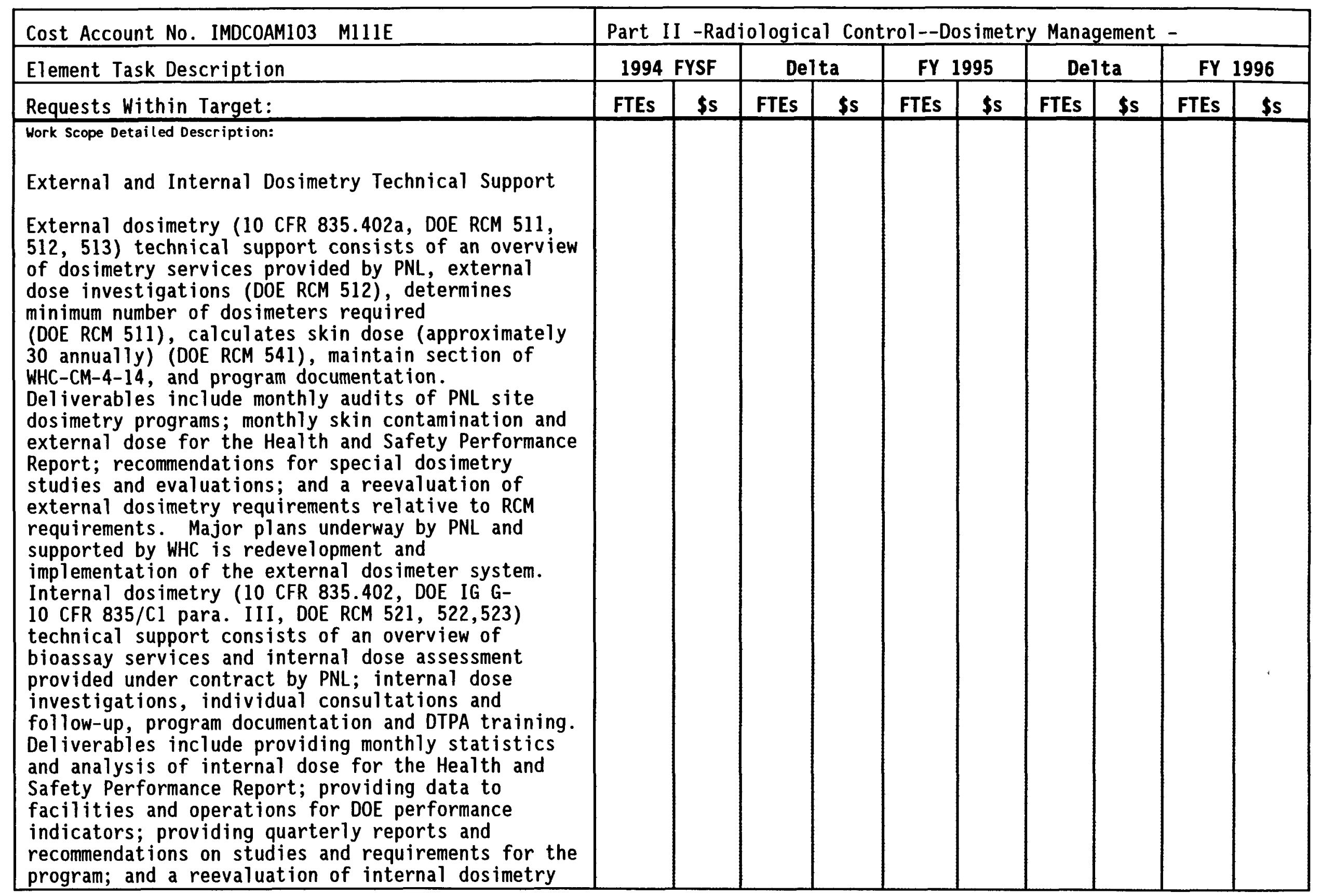




\begin{tabular}{|c|c|c|c|c|c|c|c|c|c|c|}
\hline Cost Account No. IMDCOAM103 M111E & \multicolumn{10}{|c|}{ Part II -Radiological Control--Dosimetry Management - } \\
\hline Element Task Description & \multicolumn{2}{|c|}{1994 FYSF } & \multicolumn{2}{|c|}{ Delta } & \multicolumn{2}{|c|}{ FY 1995} & \multicolumn{2}{|c|}{ Delta } & \multicolumn{2}{|c|}{ FY 1996} \\
\hline Requests Within Target: & FTES & $\$ \mathbf{s}$ & FTEs & $\$ s$ & FTEs & $\$ s$ & FTES & $\$$ s & FTEs & $\$ s$ \\
\hline WHC Labor Cost & 19.3 & 738.9 & & & 13.2 & 664.4 & & & 14.2 & 684.3 \\
\hline Total Labor & & & & & & & & & & \\
\hline $\begin{array}{l}\text { (List ONLY items that exceed } \$ 5 k \text {. [i.e., } 20 \text { Computers]) } \\
\text { Non-Labor Cost Descriptions }\end{array}$ & & & & & & & & & & \\
\hline Non-Labor Dosimetry costs & & & & & & 472.6 & & & & 486.8 \\
\hline Total Non-Labor & & & & & & 472.6 & & & & 486.8 \\
\hline Total Cost Account Within Target & 19.3 & 1177.4 & & & 13.2 & 1137.0 & & & 14.2 & 1171.1 \\
\hline
\end{tabular}




\begin{tabular}{|c|c|c|c|c|c|c|c|c|c|c|}
\hline Cost Account No. 1MDCOAM103 M111F & \multicolumn{10}{|c|}{ Part II - Radiological Control--Dosimetry- } \\
\hline Element Task Description & \multicolumn{2}{|c|}{1994 FYSF } & \multicolumn{2}{|c|}{ Delta } & \multicolumn{2}{|c|}{ FY 1995} & \multicolumn{2}{|c|}{ Delta } & \multicolumn{2}{|c|}{ FY 1996} \\
\hline Requests Within Target: & FTES & $\$ \mathbf{s}$ & FTEs & $\$ \mathbf{S}$ & FTES & $\$ s$ & FTES & $\$ \mathbf{s}$ & FTES & $\$ s$ \\
\hline \multicolumn{11}{|l|}{$\begin{array}{l}\text { Work Scope Detailed Description: } \\
\text { IRM Support of the ACES Program }\end{array}$} \\
\hline WHC Labor Cost & & & & & & 164.4 & & & 2.5 & 169.3 \\
\hline Total Labor & & & & & & & & & & \\
\hline \multicolumn{11}{|l|}{$\begin{array}{l}\text { (List ONLY items that exceed \$5K. [i.e., } 20 \text { Computers]) } \\
\text { Non-Labor Cost Descriptions }\end{array}$} \\
\hline Non Labor costs for IRM support of ACES & & & & & & 130.0 & & & & 133.9 \\
\hline Total Non-Labor & & & & & & 130.0 & & & & 133.9 \\
\hline Total Cost Account Within Target & & 95.1 & & & & 294.4 & & & 2.5 & 303.2 \\
\hline Cost Account No. 1MDCOAM110 M111J & \multicolumn{10}{|c|}{ Part II -Radiological Control - } \\
\hline Element Task Description & \multicolumn{2}{|c|}{1994 FYSF } & \multicolumn{2}{|c|}{ Delta } & \multicolumn{2}{|c|}{ FY 1995} & \multicolumn{2}{|c|}{ Delta } & \multicolumn{2}{|c|}{ FY 1996} \\
\hline Requests Within Target: & FTES & $\$$ s & FTEs & $\$$ \$ & FTES & $\$ s$ & FTES & $\$ \mathbf{s}$ & FTES & $\$ s$ \\
\hline Total Labor & & & & & & & & & & \\
\hline $\begin{array}{l}\text { (List ONLY items that exceed \$5K. [i.e., } 20 \text { Computers]) } \\
\text { Non-Labor Cost Descriptions }\end{array}$ & & & & & & & & & & \\
\hline $\begin{array}{l}\text { Non-Labor charges for PNL support of the Hanford } \\
\text { Site Radiological Control Manual }\end{array}$ & & & & & & 251.0 & & & & 258.5 \\
\hline Total Non-Labor & & & & & & 251.0 & & & & 258.5 \\
\hline Total Cost Account Within Target & & 251.0 & & & & 251.0 & & & & 258.5 \\
\hline
\end{tabular}




\begin{tabular}{|c|c|c|c|c|c|c|c|c|c|c|}
\hline \multirow{3}{*}{$\begin{array}{l}\text { Cost Account No. IMDCOAM109 M111H } \\
\text { Element Task Description } \\
\text { Requests Within Target: }\end{array}$} & \multicolumn{10}{|c|}{ Part II - CCIP and ALARA } \\
\hline & \multicolumn{2}{|c|}{1994 FYSF } & \multicolumn{2}{|c|}{ Delta } & \multicolumn{2}{|c|}{ FY 1995} & \multicolumn{2}{|c|}{ Delta } & \multicolumn{2}{|c|}{ FY 1996} \\
\hline & FTES & $\$ s$ & FTES & $\$ s$ & FTES & $\$ s$ & FTEs & $\$ s$ & FTES & $\$ s$ \\
\hline $\begin{array}{l}\text { Work scope Detailed Description: } \\
\text { The mission of the WHC ALARA Program is to provide } \\
\text { a service which coordinates and supports the } \\
\text { minimization of radiological exposures and promotes } \\
\text { contamination reduction. The ALARA activity } \\
\text { provides a variety of direct and indirect support } \\
\text { services for multiple WHC facilities, organizations } \\
\text { and WHC senior and line management. These include } \\
\text { administration through a central ized ALARA Program } \\
\text { Office (APO) which acts as the official agency for } \\
\text { ALARA policy interpretation and develops the } \\
\text { implementing program for WHC. The APO provides } \\
\text { coordination, direction and oversight for } \\
\text { implementation of facility/organizational ALARA } \\
\text { Committees, and administrates the WHC ALARA Council } \\
\text { in execution of ALARA policy and guidance to comply } \\
\text { with DOE ALARA requirements. The ALARA activity } \\
\text { also provides administration of the ALARA Awareness } \\
\text { and Performance Recognition Programs. }\end{array}$ & & & & & & & & & & \\
\hline
\end{tabular}




\begin{tabular}{|c|c|c|c|c|c|c|c|c|c|c|}
\hline \multirow{3}{*}{$\begin{array}{l}\text { Cost Account No. IMDCOAM109 M111H } \\
\text { Element Task Description } \\
\text { Requests Within Target: }\end{array}$} & \multicolumn{10}{|c|}{ Part II - CCIP and ALARA } \\
\hline & \multicolumn{2}{|c|}{1994 FYSF } & \multicolumn{2}{|c|}{ Delta } & \multicolumn{2}{|c|}{ FY 1995} & \multicolumn{2}{|c|}{ Delta } & \multicolumn{2}{|c|}{ FY 1996} \\
\hline & FTES & $\$ s$ & FTES & $\$ s$ & FTES & $\$ s$ & FTES & $\$ s$ & FTEs & \$s \\
\hline $\begin{array}{l}\text { Hork Scope Detailed Description: } \\
\text { The Contamination Control Improvement } \\
\text { Project(CCIP), as part of the WHC Occupational } \\
\text { ALARA Program and WHC Radiation Protection Program, } \\
\text { provides a centralized focal point to assist } \\
\text { facilities in a sitewide effort to improve the } \\
\text { contamination control practices at Hanford. To } \\
\text { implement the requirements of } 10 \text { CFR } 835 \text { (Subparts } \\
\text { B and E) regarding contamination control, the CCIP } \\
\text { charter includes the tracking and trending of } \\
\text { contamination on the site, the development of } \\
\text { associated sitewide performance indicators, the } \\
\text { planning and technical support for contaminated } \\
\text { area reduction, the development of sitewide map } \\
\text { depicting areas of contamination, and the } \\
\text { performance of assessments regarding contamination } \\
\text { control work practices. The focus of CCIP is to } \\
\text { help maintain a safe workplace by minimizing the } \\
\text { occupational risks that result from the interaction } \\
\text { between people and contamination and to maintain } \\
\text { contamination under good radiological control. The } \\
\text { following resources, as a minimum, are required for } \\
\text { the upcoming fiscal year. }\end{array}$ & & & & & & & & & & \\
\hline
\end{tabular}




\begin{tabular}{|c|c|c|c|c|c|c|c|c|c|c|}
\hline Cost Account No. 1MDCOAM109 M111H & \multicolumn{10}{|c|}{ Part II - CCIP and ALARA } \\
\hline Element Task Description & \multicolumn{2}{|c|}{1994 FYSF } & \multicolumn{2}{|c|}{ Delta } & \multicolumn{2}{|c|}{ FY 1995} & \multicolumn{2}{|c|}{ Delta } & \multicolumn{2}{|c|}{ FY 1996} \\
\hline Requests Within Target: & FTES & $\$ s$ & FTES & $\$ s$ & FTEs & $\$ s$ & FTES & $\$ s$ & FTES & $\$ s$ \\
\hline WHC Labor Cost & 5.5 & 347.7 & & & 5.0 & 330.6 & & & 5.0 & 340.5 \\
\hline Total Labor & & & & & & & & & & \\
\hline $\begin{array}{l}\text { (List ONLY items that exceed } \$ 5 k \text {. [i.e., } 20 \text { Computers]) } \\
\text { Non-Labor Cost Descriptions }\end{array}$ & & & & & & & & & & \\
\hline Non-Labor cost of the ALARA Program & & & & & & 178.8 & & & & 184.2 \\
\hline Total Non-Labor & & & & & & 178.8 & & & & 184.2 \\
\hline Total Cost Account Within Target & 5.5 & 347.7 & & & 5.0 & 509.4 & & & 5.0 & 524.7 \\
\hline Total Cost Account for Dosimetry Pool Within Target & 21.3 & 13015.0 & & & 22.5 & 12743.5 & & 381.8 & 22.5 & 13125.3 \\
\hline
\end{tabular}




\begin{tabular}{|c|c|c|c|c|}
\hline \multicolumn{3}{|c|}{ 2.B.1 Milestone List } & $\begin{array}{c}\text { West inghouse Hanford Company } \\
\text { Radiological Control } \\
6.7 .2 .4 \\
\end{array}$ & $\begin{array}{l}\text { FY } 1995 \\
\text { Site Support Program Plan } \\
\text { Date Prepared:August } 30,1994\end{array}$ \\
\hline \multicolumn{2}{|c|}{ Milestone } & \multirow{2}{*}{$\begin{array}{c}\text { WBS } \\
\text { Number }\end{array}$} & \multirow{2}{*}{ Milestone Description } & \multirow{2}{*}{$\begin{array}{l}\text { Scheduled } \\
\text { Completion } \\
\text { Date } \\
\end{array}$} \\
\hline Type & Number & & & \\
\hline RL & RAD-95-001 & 6.7.2.4 & Quarterly Report of Radiological Performance Indicators & $05 / 31 / 95$ \\
\hline RL & RAD-95-002 & 6.7.2.4 & Quarterly Report of Radiological Performance Indicators & $08 / 31 / 95$ \\
\hline $\mathrm{RL}$ & RAD - 95-003 & 6.7.2.4 & Annual Report of Radiological Performance Indicators & 03/31/95 \\
\hline RL & RAD-95-004 & 6.7.2.4 & Bi-monthly Program Review with DOE & $11 / 30 / 94$ \\
\hline $\mathrm{RL}$ & RAD-95-005 & 6.7.2.4 & Bi-monthly Program Review with DOE & $01 / 31 / 95$ \\
\hline $\mathrm{RL}$ & RAD-95-006 & 6.7.2.4 & Bi-monthly Program Review with DOE & $03 / 31 / 95$ \\
\hline RL & RAD-95-007 & 6.7.2.4 & Bi-monthly Program Review with DOE & 05/31/95 \\
\hline RL & RAD-95-008 & 6.7.2.4 & Bi-monthty Program Review with DOE & $07 / 31 / 95$ \\
\hline $\mathrm{RL}$ & RAD-95-009 & 6.7.2.4 & Bi-monthly Program Review with DOE & $09 / 30 / 95$ \\
\hline RL & RAD-95-010 & 6.7.2.4 & Perform ALARA Program Review with DOE-RL & $12 / 30 / 94$ \\
\hline RL & RAD - 95-011 & 6.7.2.4 & Perform ALARA Program Review with DOE-RL & $03 / 31 / 95$ \\
\hline RL & RAD-95-012 & 6.7.2.4 & Perform ALARA Program Review with DOE-RL & $06 / 30 / 95$ \\
\hline RL & RAD-95-013 & 6.7.2.4 & Perform ALARA Program Review with DOE-RL & $09 / 29 / 95$ \\
\hline $\mathrm{RL}$ & RAD-95-014 & 6.7.2.4 & Report of UHC FY 1995 ALARA Performance Goals & $11 / 30 / 94$ \\
\hline $\mathrm{RL}$ & RAD-95-015 & 6.7.2.4 & Summary Report for CY1994 ALARA Goals & $04 / 28 / 95$ \\
\hline $\mathrm{RL}$ & RAD-95-016 & 6.7.2.4 & Submittal of RPP to DOE-RL & $12 / 31 / 94$ \\
\hline RL & RAD-95-017 & 6.7.2.4 & Year end report of DOE RadCon Manual Compl iance & $12 / 31 / 94$ \\
\hline RL & RAD-95-018 & 6.7.2.4 & Report on status of HSRCM Rev. 1.0 compliance actions & 09/30/95 \\
\hline
\end{tabular}




\begin{tabular}{|c|c|c|c|c|}
\hline \multicolumn{3}{|c|}{ 2.B.1 Milestone List } & $\begin{array}{c}\text { Westinghouse Hanford Company } \\
\text { Radiological Control } \\
6.7 .2 .4\end{array}$ & $\begin{array}{l}\text { FY } 1995 \\
\text { Site Support Program Plan } \\
\text { Date Prepared:August 30, } 1994\end{array}$ \\
\hline \multicolumn{2}{|c|}{ Milestone } & \multirow{2}{*}{$\begin{array}{c}\text { WBS } \\
\text { Number }\end{array}$} & \multirow{2}{*}{ Milestone Description } & Scheduled \\
\hline Type & Number & & & $\begin{array}{l}\text { Date } \\
\text { Daimplection } \\
\end{array}$ \\
\hline RL & RAD-95-019 & 6.7.2.4 & Complete all actions for Rev. 1.0 of the DOE RadCon Manual & $03 / 30 / 95$ \\
\hline RL & RAD - $95-020$ & 6.7.2.4 & Reduce No. of people in external dosimetry program & $09 / 30 / 95$ \\
\hline $\mathrm{RL}$ & RAD-95-021 & 6.7.2.4 & Reduce No. of people in internal dosimetry program & $09 / 30 / 95$ \\
\hline RL & RAD-95-022 & 6.7.2.4 & Radiological Emergency Preparedness Drills & $12 / 31 / 94$ \\
\hline RL & RAD - $95-023$ & 6.7.2.4 & Radiological Emergency Preparedness Drills & $06 / 30 / 95$ \\
\hline WHC & RAD-95-024 & 6.7.2.4 & Radiological Emergency Preparedness Drills & $03 / 31 / 95$ \\
\hline WHC & RAD-95-025 & 6.7.2.4 & Radiological Emergency Preparedness Drills & $09 / 30 / 95$ \\
\hline
\end{tabular}




\begin{tabular}{|c|c|c|}
\hline $\begin{array}{c}\text { Milestone Description } \\
\text { Sheet }\end{array}$ & $\begin{array}{l}\text { Westinghouse Hanford Company } \\
6.7 .2 .4 \text { Radiological Control }\end{array}$ & $\begin{array}{c}\text { FY } 1995 \\
\text { SSPP }\end{array}$ \\
\hline
\end{tabular}

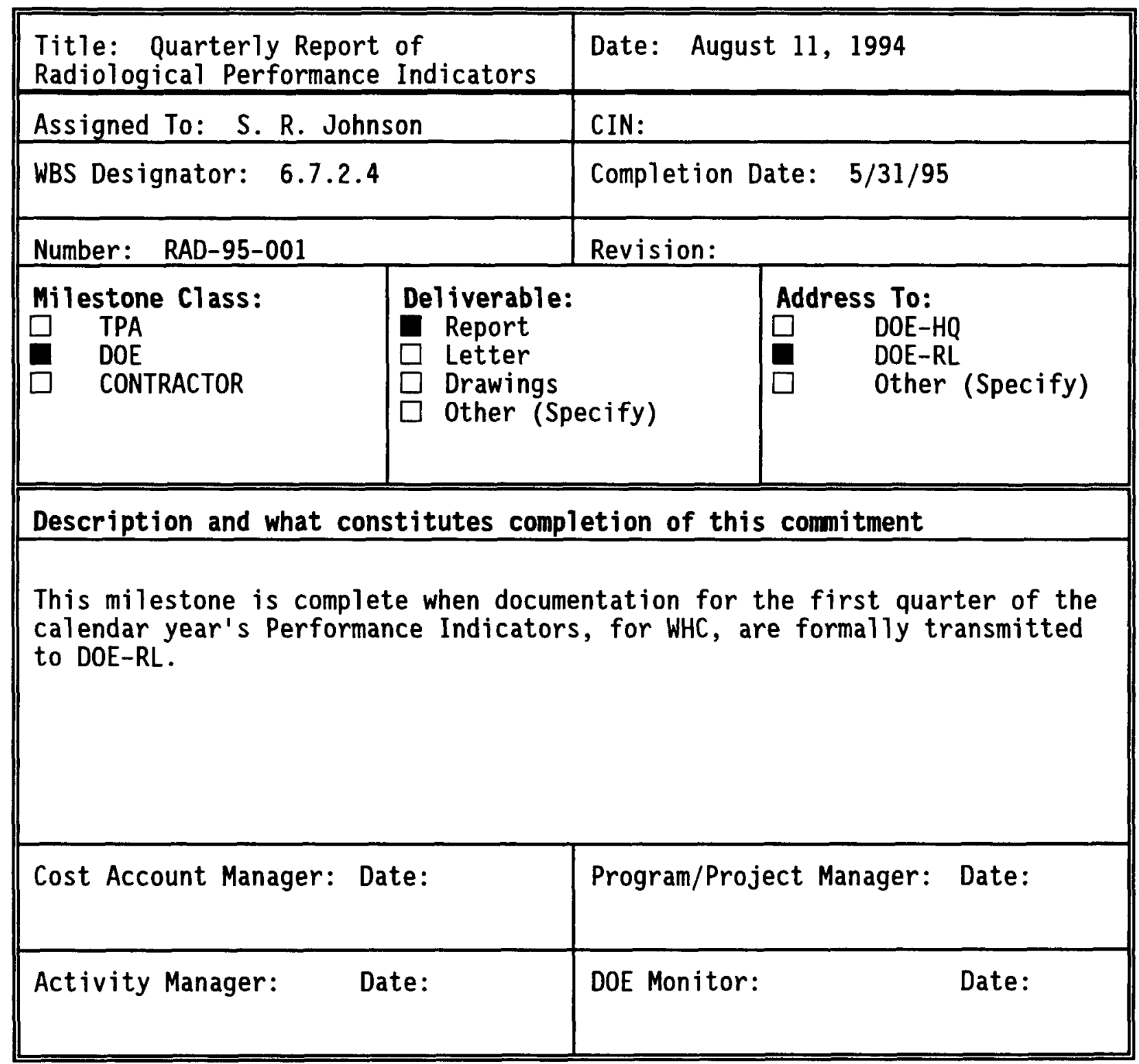




\begin{tabular}{|c|c|c|}
\hline $\begin{array}{c}\text { Milestone Description } \\
\text { Sheet }\end{array}$ & $\begin{array}{l}\text { Westinghouse Hanford Company } \\
\text { 6.7.2.4 Radiological Control }\end{array}$ & $\begin{array}{c}\text { FY } 1995 \\
\text { SSPP }\end{array}$ \\
\hline
\end{tabular}

\begin{tabular}{|c|c|c|c|}
\hline \multicolumn{2}{|c|}{$\begin{array}{l}\text { Title: Quarterly Report of } \\
\text { Radiological Performance Indicators }\end{array}$} & \multicolumn{2}{|c|}{ Date: August 11, 1994} \\
\hline \multicolumn{2}{|c|}{ Assigned To: S. R. Johnson } & \multicolumn{2}{|l|}{ CIN: } \\
\hline \multicolumn{2}{|l|}{ WBS Designator: 6.7 .2 .4} & \multicolumn{2}{|c|}{ Completion Date: $\quad 8 / 31 / 95$} \\
\hline \multicolumn{2}{|l|}{ Number: RAD-95-002 } & \multicolumn{2}{|l|}{ Revision: } \\
\hline $\begin{array}{l}\text { Milestone class: } \\
\quad \text { TPA } \\
\text { DOE } \\
\quad \text { CONTRACTOR }\end{array}$ & \multicolumn{2}{|c|}{$\begin{array}{l}\text { Deliverable: } \\
\text { Report } \\
\square \text { Letter } \\
\square \text { Drawings } \\
\square \text { Other (Specify) }\end{array}$} & 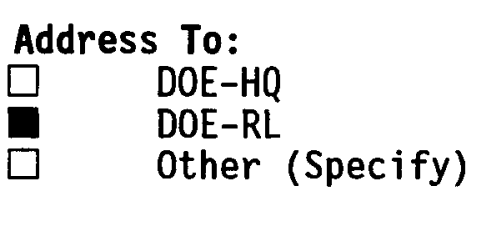 \\
\hline \multicolumn{4}{|c|}{ Description and what constitutes completion of this commitment } \\
\hline \multicolumn{4}{|c|}{$\begin{array}{l}\text { This milestone is complete when documentation for the second quarter of the } \\
\text { calendar year's Performance Indicators, for WHC, are formally transmitted } \\
\text { to DOE-RL. }\end{array}$} \\
\hline \multicolumn{2}{|c|}{ Cost Account Manager: Date: } & \multicolumn{2}{|c|}{ Program/Project Manager: Date: } \\
\hline Activity Manager: & ite: & DOE Monitor: & Date: \\
\hline
\end{tabular}




\begin{tabular}{|c|c|c|}
\hline $\begin{array}{c}\text { Milestone Description } \\
\text { Sheet }\end{array}$ & $\begin{array}{l}\text { Westinghouse Hanford Company } \\
\text { 6.7.2.4 Radiological Control }\end{array}$ & $\begin{array}{c}\text { FY } 1995 \\
\text { SSPP }\end{array}$ \\
\hline
\end{tabular}

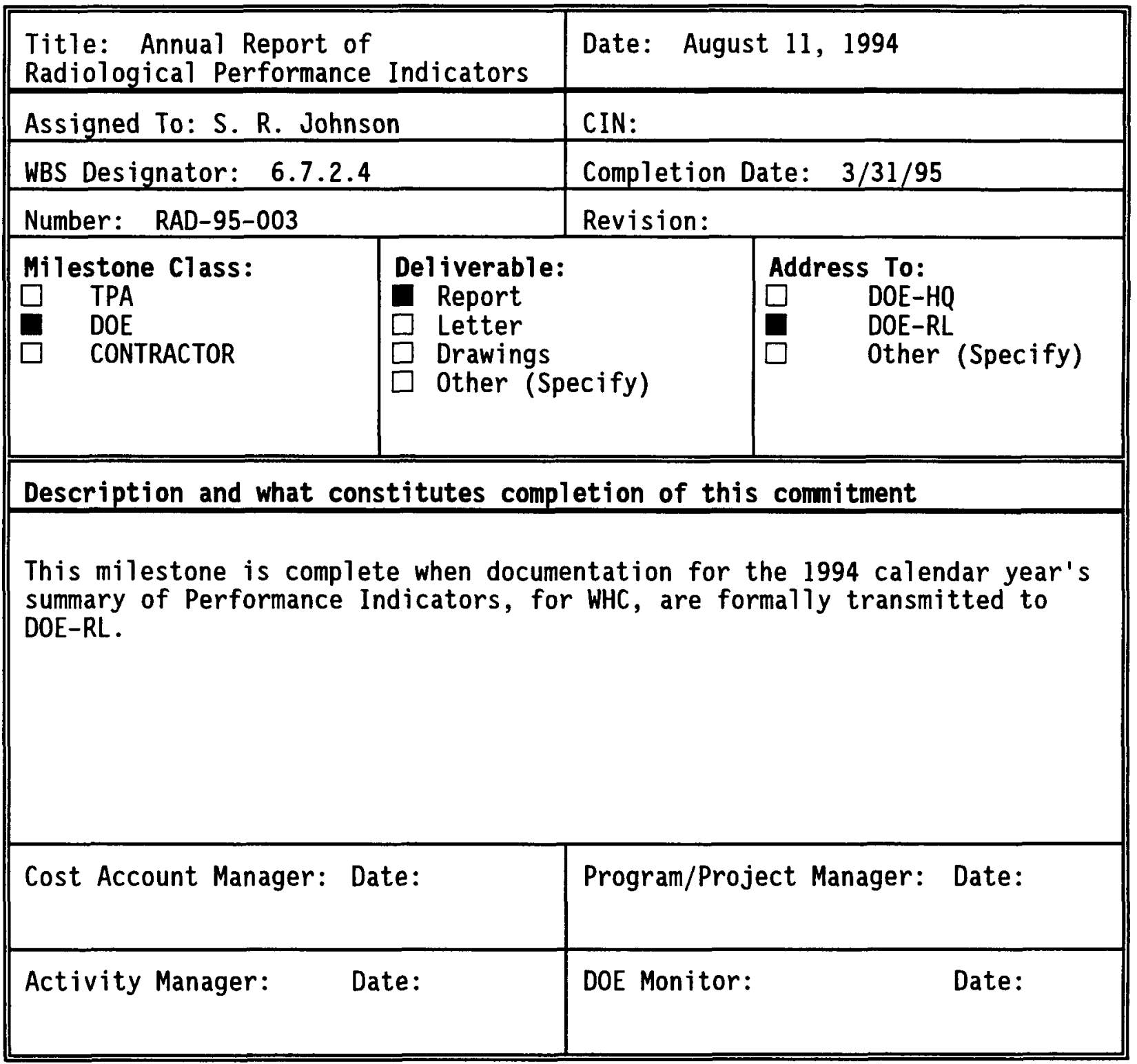




\begin{tabular}{|c|c|c|}
\hline $\begin{array}{c}\text { Milestone Description } \\
\text { Sheet }\end{array}$ & $\begin{array}{c}\text { Westinghouse Hanford Company } \\
6.7 .2 .4 \text { Radiological Control }\end{array}$ & $\begin{array}{c}\text { FY } 1995 \\
\text { SSPP }\end{array}$ \\
\hline
\end{tabular}

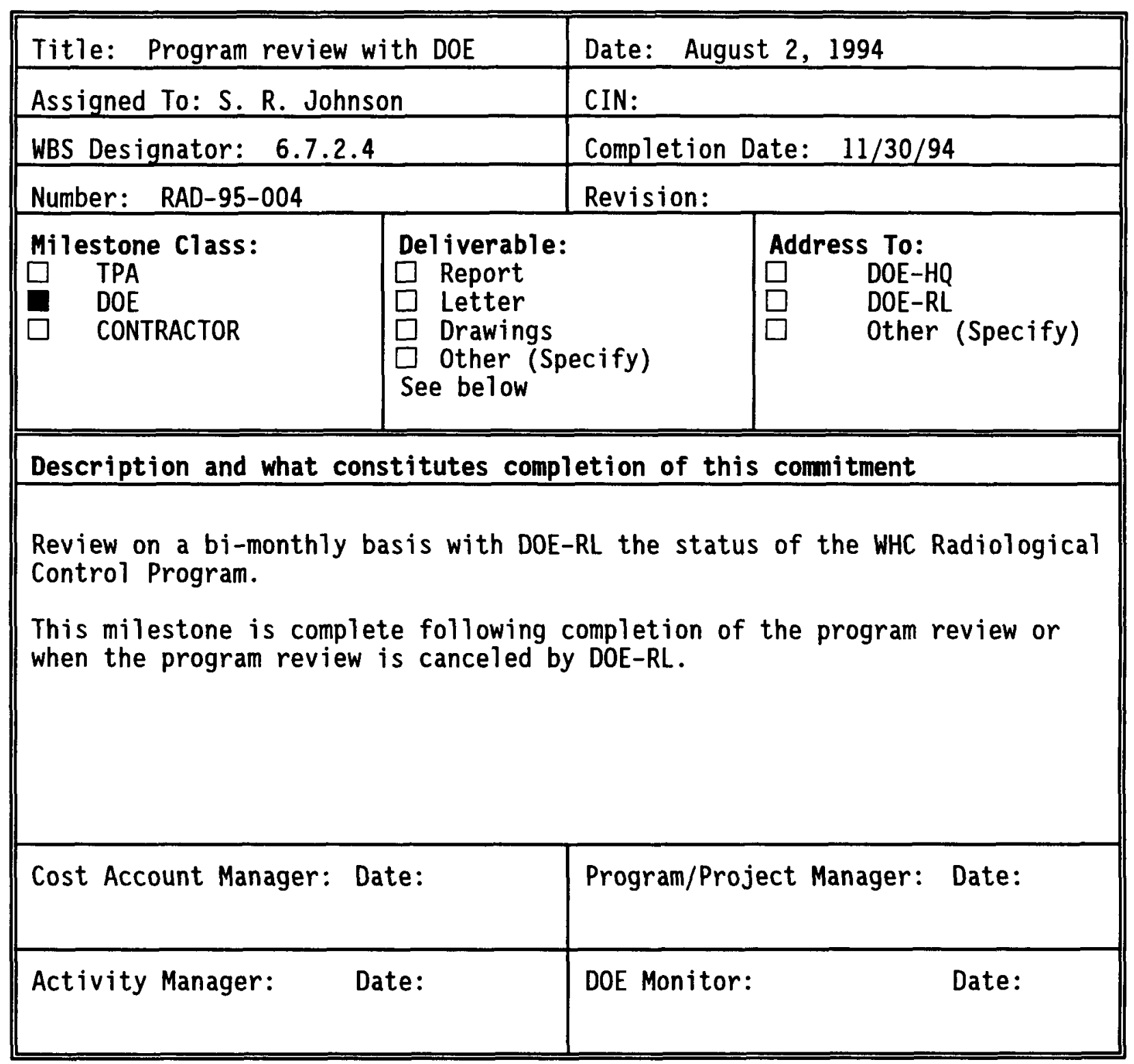




\begin{tabular}{|c|c|c|}
\hline $\begin{array}{c}\text { Milestone Description } \\
\text { Sheet }\end{array}$ & $\begin{array}{l}\text { Westinghouse Hanford Company } \\
6.7 .2 .4 \text { Radiological Control }\end{array}$ & $\begin{array}{c}\text { FY } 1995 \\
\text { SSPP }\end{array}$ \\
\hline
\end{tabular}

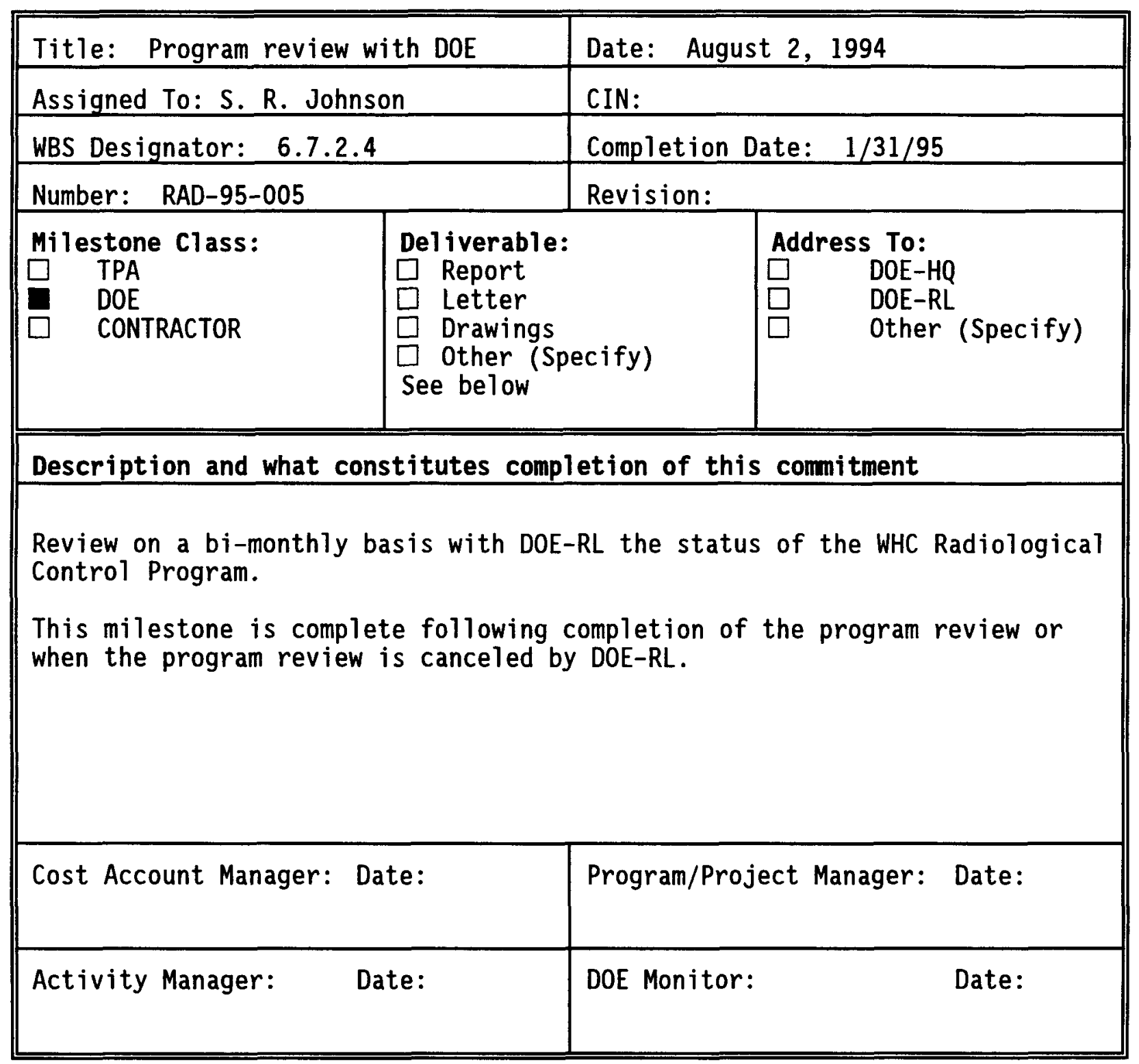




\begin{tabular}{|c|c|c|}
\hline $\begin{array}{c}\text { Milestone Description } \\
\text { Sheet }\end{array}$ & $\begin{array}{c}\text { Westinghouse Hanford Company } \\
6.7 .2 .4 \text { Radiological Control }\end{array}$ & $\begin{array}{c}\text { FY } 1995 \\
\text { SSPP }\end{array}$ \\
\hline
\end{tabular}

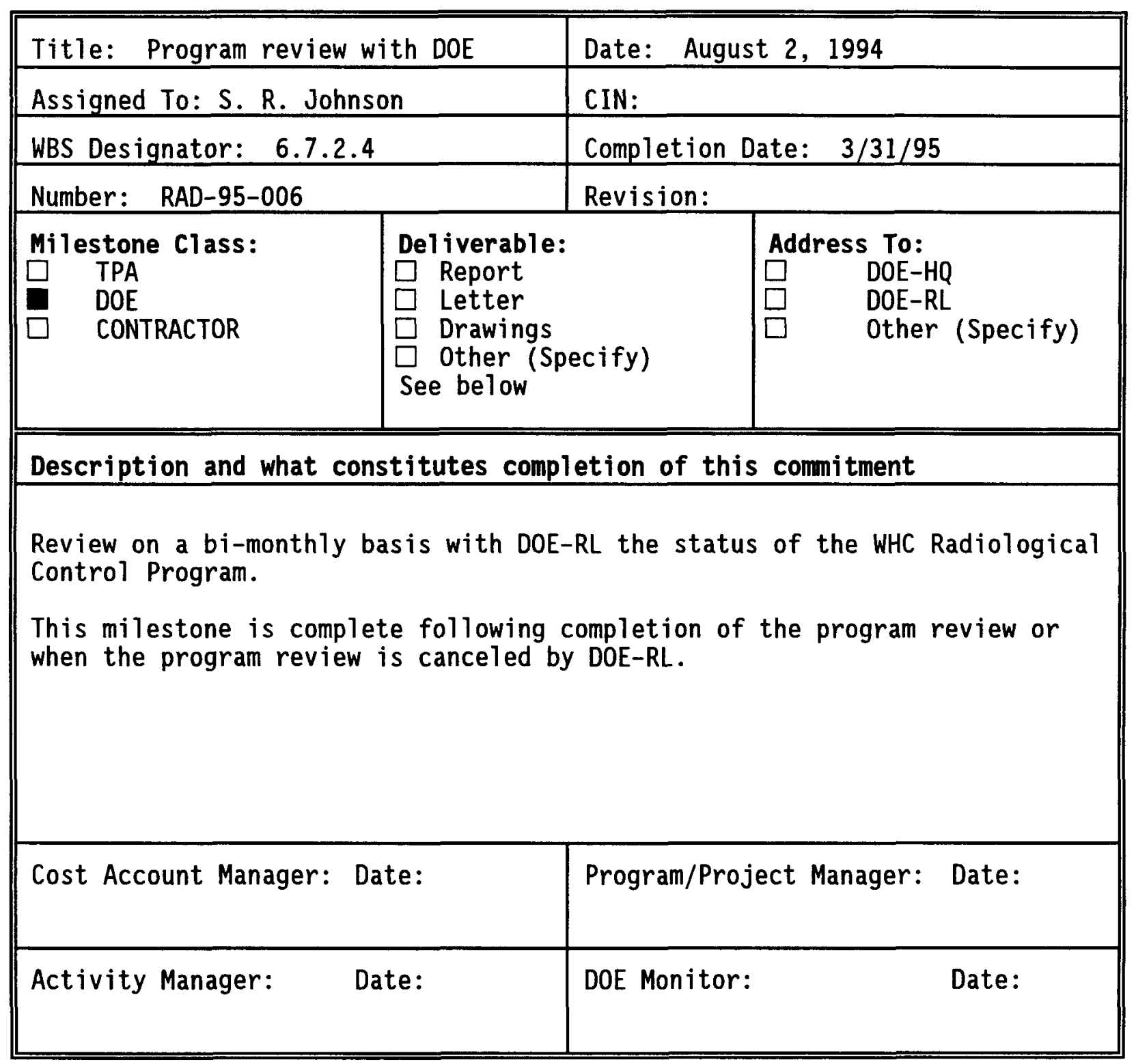




\begin{tabular}{|c|c|c|}
\hline $\begin{array}{c}\text { Milestone Description } \\
\text { Sheet }\end{array}$ & $\begin{array}{c}\text { Westinghouse Hanford Company } \\
6.7 .2 .4 \text { Radiological Control }\end{array}$ & $\begin{array}{c}\text { FY } 1995 \\
\text { SSPP }\end{array}$ \\
\hline
\end{tabular}

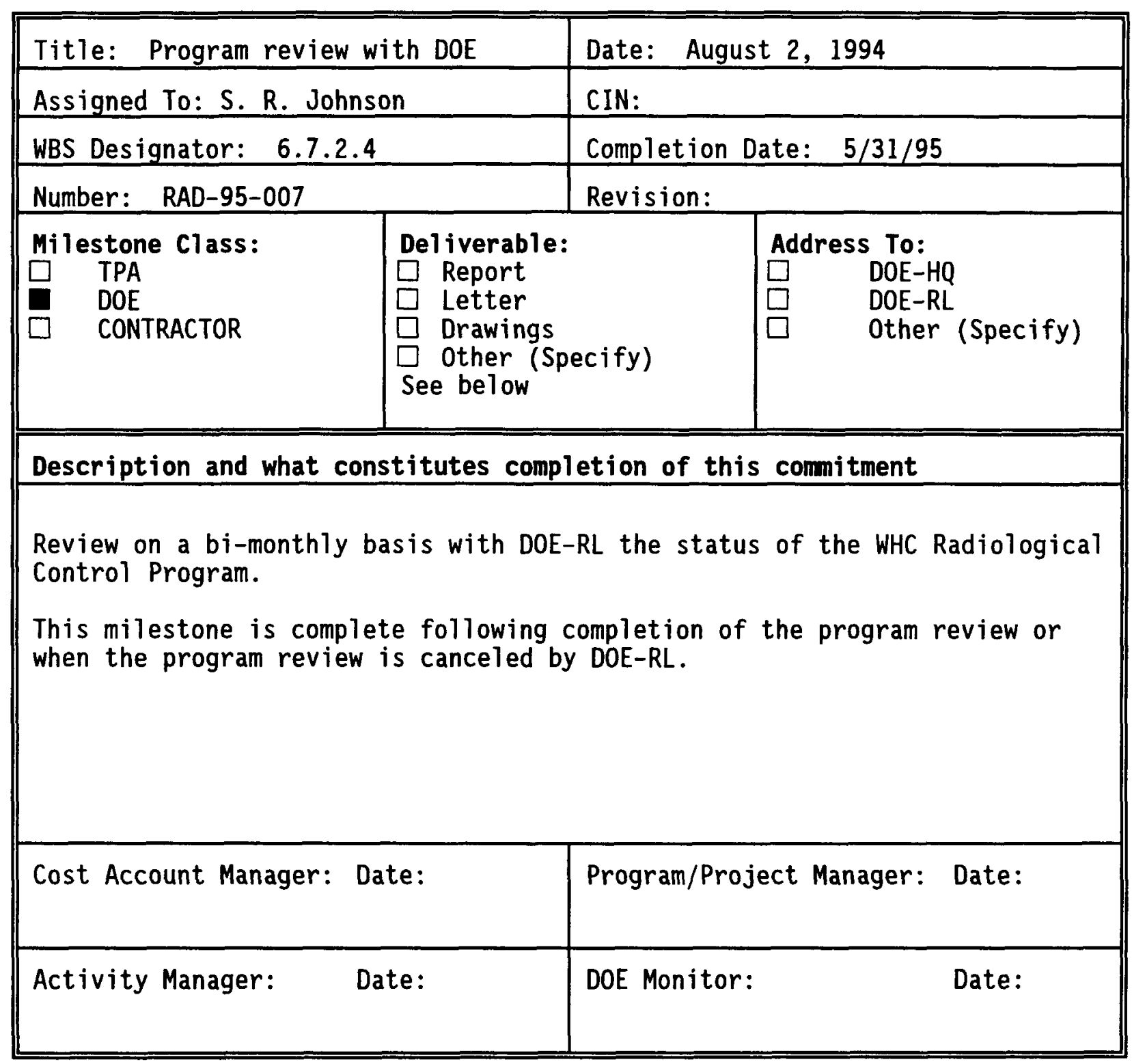




\begin{tabular}{|c|c|c|}
\hline $\begin{array}{c}\text { Milestone Description } \\
\text { Sheet }\end{array}$ & $\begin{array}{c}\text { Westinghouse Hanford Company } \\
6.7 .2 .4 \text { Radiological Control }\end{array}$ & $\begin{array}{c}\text { FY } 1995 \\
\text { SSPP }\end{array}$ \\
\hline
\end{tabular}

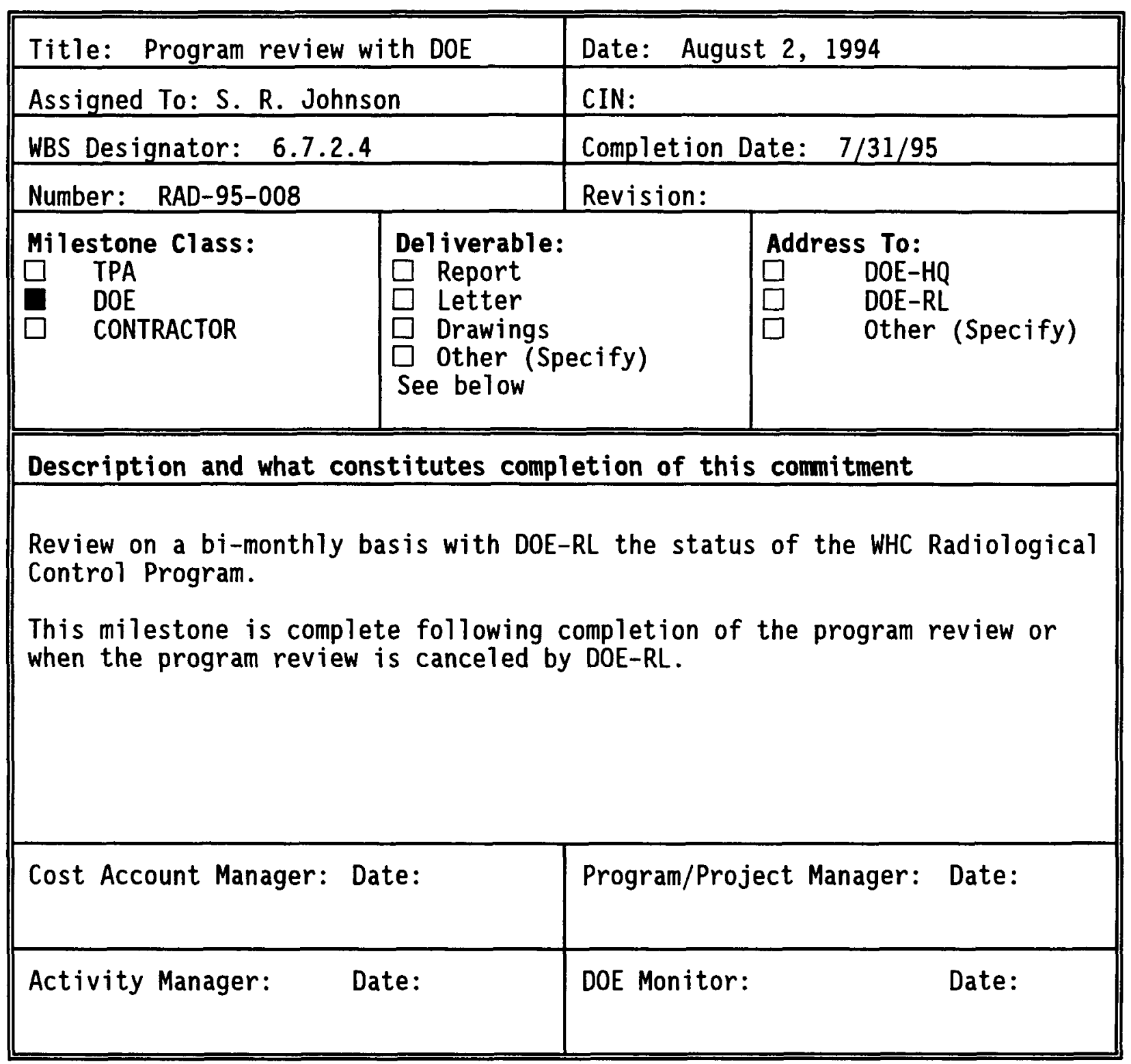




\begin{tabular}{|c|c|c|}
\hline $\begin{array}{c}\text { Milestone Description } \\
\text { Sheet }\end{array}$ & $\begin{array}{c}\text { Westinghouse Hanford Company } \\
6.7 .2 .4 \text { Radiological Control }\end{array}$ & $\begin{array}{c}\text { FY } 1995 \\
\text { SSPP }\end{array}$ \\
\hline
\end{tabular}

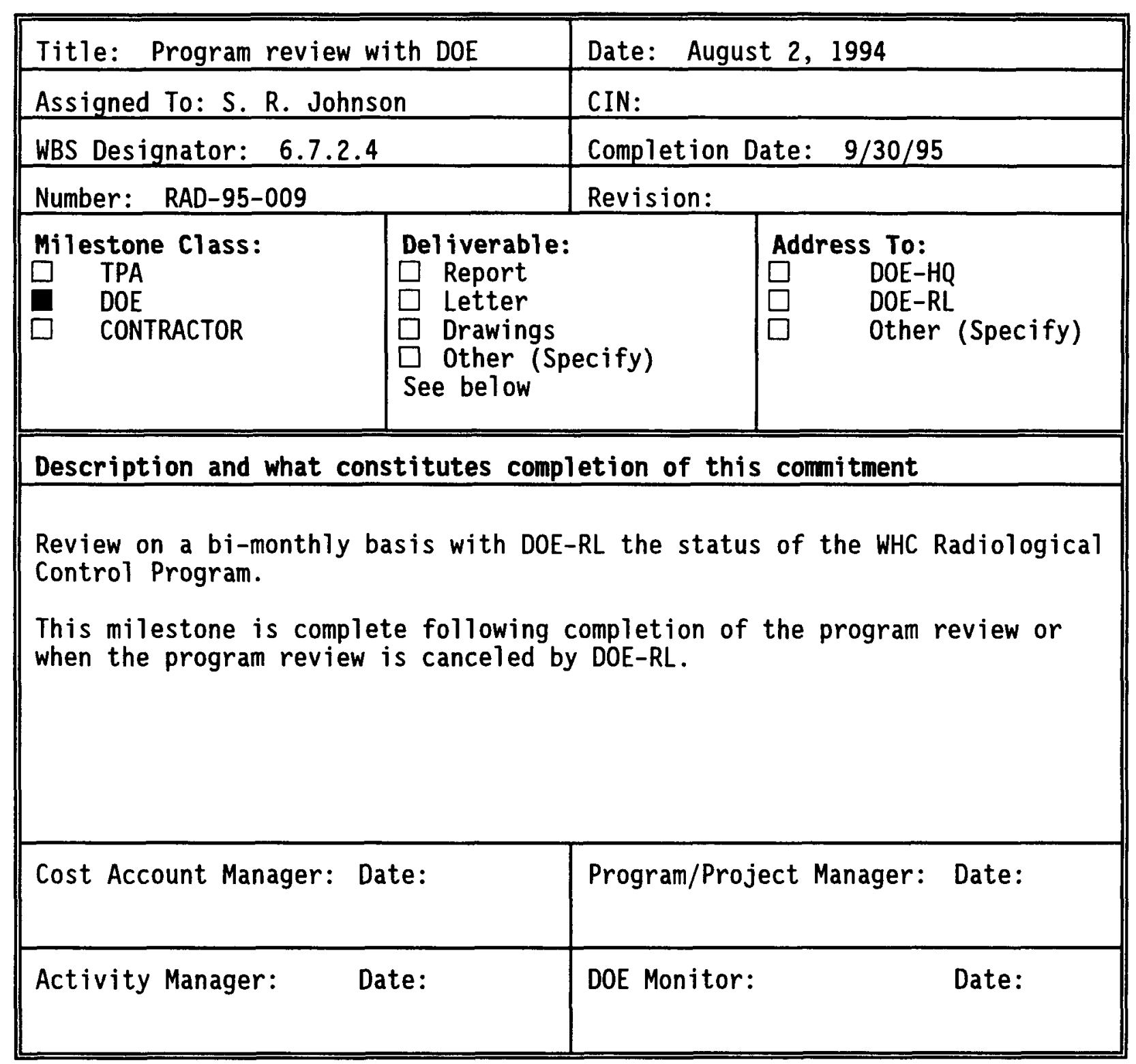




\begin{tabular}{|c|c|c|}
\hline $\begin{array}{c}\text { Milestone Description } \\
\text { Sheet }\end{array}$ & $\begin{array}{c}\text { Westinghouse Hanford Company } \\
6.7 .2 .4 \text { Radiological Control }\end{array}$ & $\begin{array}{c}\text { FY } 1995 \\
\text { SSPP }\end{array}$ \\
\hline
\end{tabular}

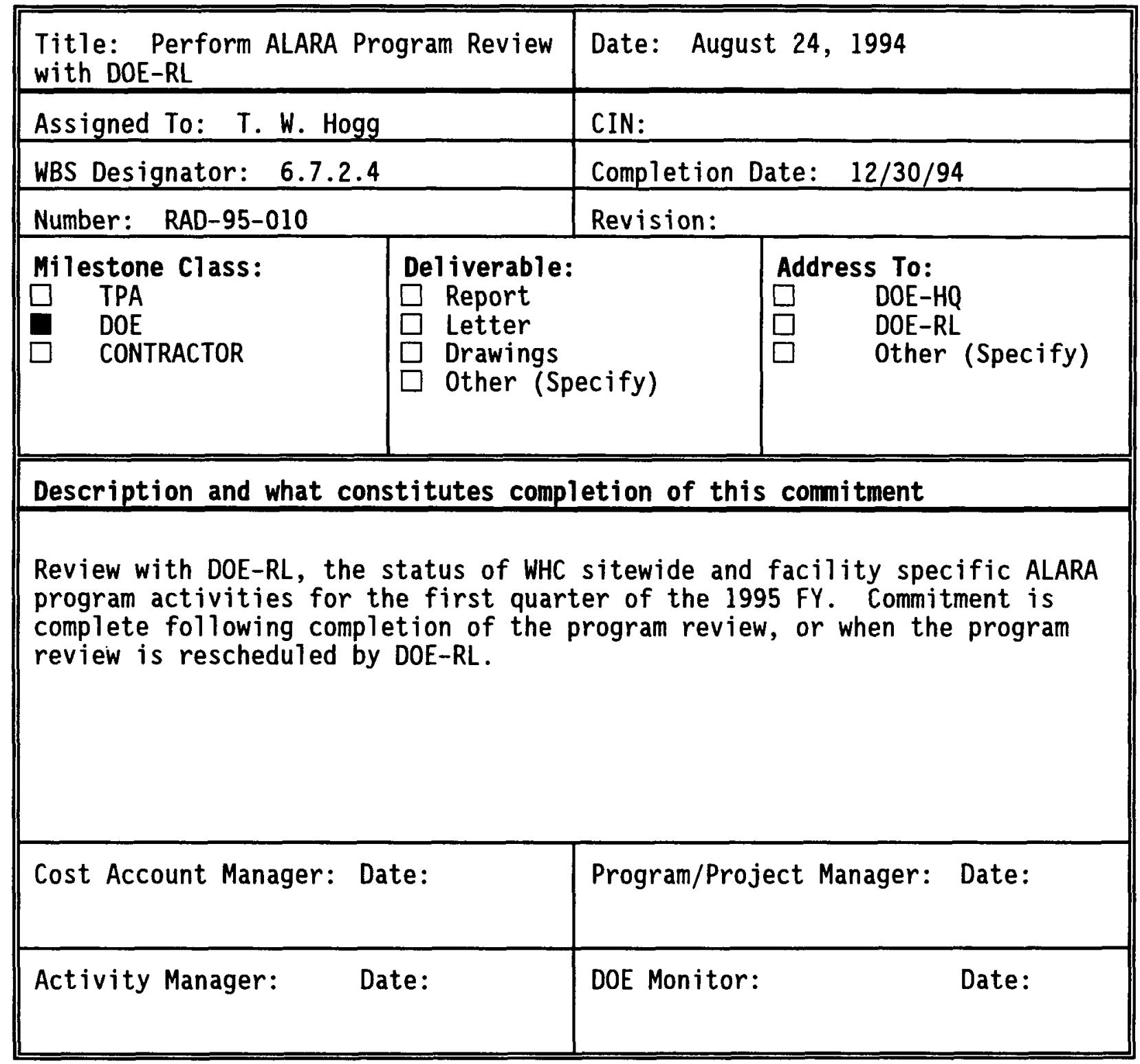




\begin{tabular}{|c|c|c|}
\hline $\begin{array}{c}\text { Milestone Description } \\
\text { Sheet }\end{array}$ & $\begin{array}{l}\text { Westinghouse Hanford Company } \\
6.7 .2 .4 \text { Radiological Control }\end{array}$ & $\begin{array}{c}\text { FY } 1995 \\
\text { SSPP }\end{array}$ \\
\hline
\end{tabular}

\begin{tabular}{|c|c|c|c|}
\hline \multicolumn{2}{|c|}{$\begin{array}{l}\text { Title: Perform ALARA Program Review } \\
\text { with DOE-RL }\end{array}$} & \multicolumn{2}{|c|}{ Date: August 24, 1994} \\
\hline \multicolumn{2}{|c|}{ Assigned To: T. W. Hogg } & \multicolumn{2}{|l|}{ CIN: } \\
\hline \multicolumn{2}{|c|}{ WBS Designator: 6.7 .2 .4} & \multicolumn{2}{|c|}{ Completion Date: $3 / 31 / 95$} \\
\hline \multicolumn{2}{|c|}{ Number: $\operatorname{RAD}-95-011$} & \multicolumn{2}{|l|}{ Revision: } \\
\hline $\begin{array}{l}\text { Milestone Class: } \\
\square \quad \text { TPA } \\
\text { DOE } \\
\square \quad \text { CONTRACTOR }\end{array}$ & \multicolumn{2}{|c|}{$\begin{array}{l}\text { Deliverable: } \\
\square \text { Report } \\
\square \text { Letter } \\
\square \text { Drawings } \\
\square \text { Other (Specify) }\end{array}$} & \begin{tabular}{|l} 
Address To: \\
$\square$ \\
$\square \quad$ DOE-HQ \\
$\square \quad$ DOE-RL \\
$\square \quad$ Other (Specify)
\end{tabular} \\
\hline \multicolumn{4}{|c|}{ Description and what constitutes completion of this commitment } \\
\hline \multicolumn{4}{|c|}{$\begin{array}{l}\text { Review with DOE-RL, the status of WHC sitewide and facility specific ALARA } \\
\text { program activities for the second quarter of the } 1995 \mathrm{FY} \text {. Commitment is } \\
\text { complete following completion of the program review, or when the program } \\
\text { review is rescheduled by DOE-RL. }\end{array}$} \\
\hline \multicolumn{2}{|c|}{ Cost Account Manager: Date: } & \multicolumn{2}{|c|}{ Program/Project Manager: Date: } \\
\hline Activity Manager & ate: & DOE Monitor: & Date: \\
\hline
\end{tabular}




\begin{tabular}{|c|c|c|}
\hline $\begin{array}{c}\text { Milestone Description } \\
\text { Sheet }\end{array}$ & $\begin{array}{c}\text { Westinghouse Hanford Company } \\
6.7 .2 .4 \text { Radiological Control }\end{array}$ & $\begin{array}{c}\text { FY } 1995 \\
\text { SSPP }\end{array}$ \\
\hline
\end{tabular}

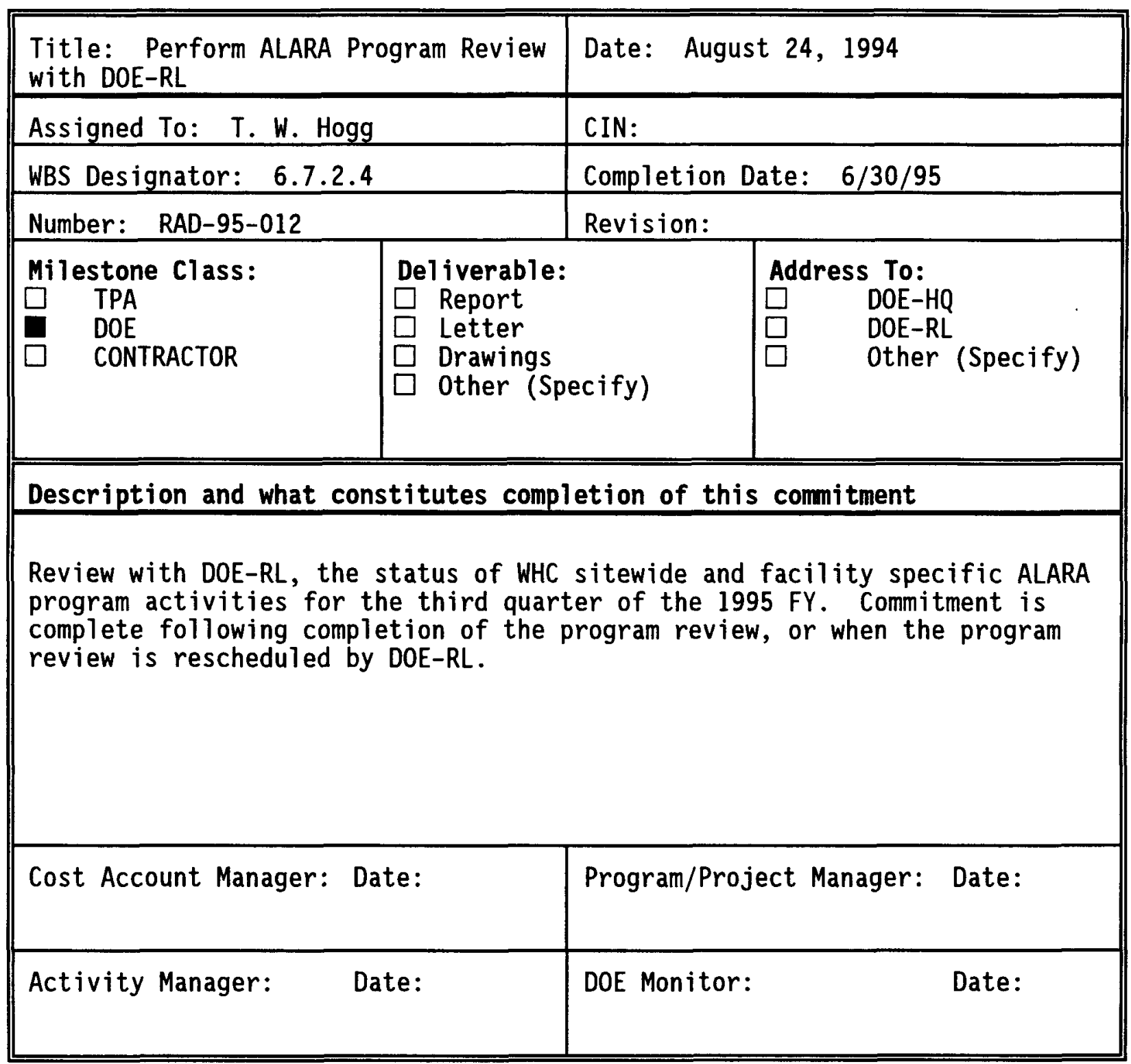




\begin{tabular}{||c|c|c|}
\hline $\begin{array}{c}\text { Milestone Description } \\
\text { Sheet }\end{array}$ & $\begin{array}{c}\text { Westinghouse Hanford Company } \\
6.7 .2 .4 \text { Radiological Control }\end{array}$ & $\begin{array}{c}\text { FY } 1995 \\
\text { SSPP }\end{array}$ \\
\hline
\end{tabular}

\begin{tabular}{|c|c|c|c|}
\hline \multicolumn{2}{|c|}{$\begin{array}{l}\text { Title: Perform ALARA Program Review } \\
\text { with DOE-RL }\end{array}$} & \multicolumn{2}{|c|}{ Date: August 24, 1994} \\
\hline \multicolumn{2}{|c|}{ Assigned To: T. W. Hogg } & \multicolumn{2}{|l|}{ CIN: } \\
\hline \multicolumn{2}{|c|}{ WBS Designator: $\quad 6 \cdot 7 \cdot 2.4$} & \multicolumn{2}{|c|}{ Completion Date: $9 / 29 / 95$} \\
\hline \multicolumn{2}{|c|}{ Number: RAD-95-013 } & \multicolumn{2}{|l|}{ Revision: } \\
\hline $\begin{array}{l}\text { Milestone Class: } \\
\square \quad \text { TPA } \\
\text { DOE } \\
\square \quad \text { CONTRACTOR }\end{array}$ & \multicolumn{2}{|c|}{$\begin{array}{l}\text { Del iverable: } \\
\square \text { Report } \\
\square \text { Letter } \\
\square \text { Drawings } \\
\square \text { Other (Specify) }\end{array}$} & $\begin{array}{l}\text { Address To: } \\
\begin{array}{l}\text { DOE-HQ } \\
\square \\
\square \quad \text { DOE-RL } \\
\square \quad \text { Other (Specify) }\end{array}\end{array}$ \\
\hline \multicolumn{4}{|c|}{ Description and what constitutes completion of this commitment } \\
\hline \multicolumn{4}{|c|}{$\begin{array}{l}\text { Review with DOE-RL, the status of WHC sitewide and facility specific ALARA } \\
\text { program activities for the fourth quarter of the } 1995 \mathrm{FY} \text {. Commitment is } \\
\text { complete following completion of the program review, or when the program } \\
\text { review is rescheduled by DOE-RL. }\end{array}$} \\
\hline \multicolumn{2}{|c|}{ Cost Account Manager: Date: } & \multicolumn{2}{|c|}{ Program/Project Manager: Date: } \\
\hline Activity Manager & te: & DOE Monitor: & Date: \\
\hline
\end{tabular}




\begin{tabular}{|c|c|c|}
\hline $\begin{array}{c}\text { Milestone Description } \\
\text { Sheet }\end{array}$ & $\begin{array}{l}\text { Westinghouse Hanford Company } \\
6.7 .2 .4 \text { Radiological Control }\end{array}$ & $\begin{array}{c}\text { FY } 1995 \\
\text { SSPP }\end{array}$ \\
\hline
\end{tabular}

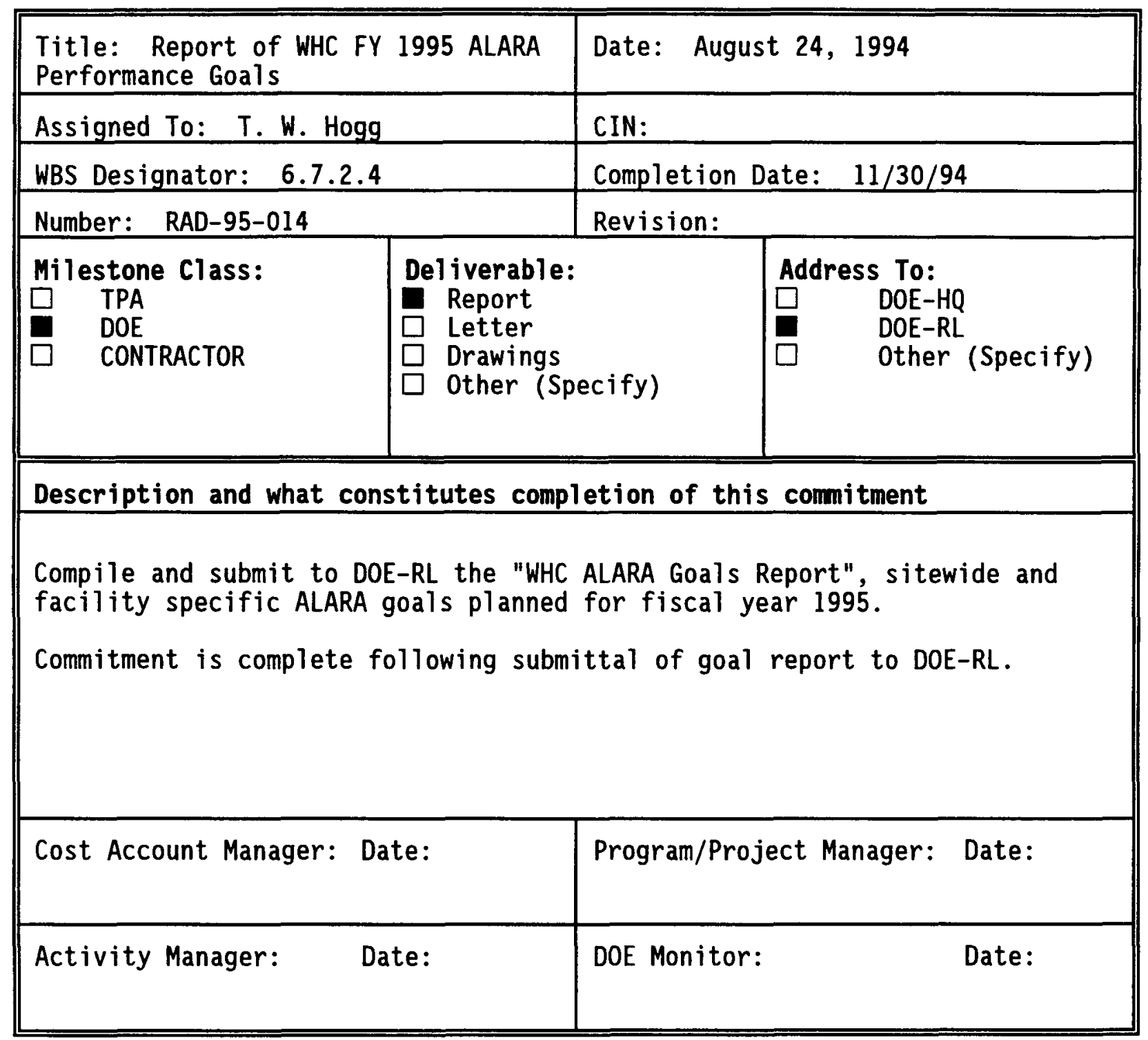




\begin{tabular}{|c|c|c|}
\hline $\begin{array}{c}\text { Milestone Description } \\
\text { Sheet }\end{array}$ & $\begin{array}{l}\text { Westinghouse Hanford Company } \\
6.7 .2 .4 \text { Radiological Control }\end{array}$ & $\begin{array}{c}\text { FY } 1995 \\
\text { SSPP }\end{array}$ \\
\hline
\end{tabular}

\begin{tabular}{|c|c|c|c|}
\hline \multicolumn{2}{|c|}{$\begin{array}{l}\text { Title: Summary Report for } \mathrm{CY} 1994 \\
\text { ALARA Goals }\end{array}$} & \multicolumn{2}{|c|}{ Date: August 24, 1994} \\
\hline \multicolumn{2}{|c|}{ Assigned To: T. W. Hogg } & \multicolumn{2}{|l|}{ CIN: } \\
\hline \multicolumn{2}{|c|}{ WBS Designator: 6.7 .2 .4} & \multicolumn{2}{|c|}{ Completion Date: $04 / 28 / 95$} \\
\hline \multicolumn{2}{|c|}{ Number: RAD-95-015 } & \multicolumn{2}{|c|}{ Revision: } \\
\hline $\begin{array}{l}\text { Milestone Class: } \\
\square \quad \text { TPA } \\
\text { DOE } \\
\square \quad \text { CONTRACTOR }\end{array}$ & \multicolumn{2}{|c|}{$\begin{array}{l}\text { Deliverable: } \\
\text { Report } \\
\square \text { Letter } \\
\square \text { Drawings } \\
\square \text { Other (Specify) }\end{array}$} & $\begin{array}{l}\text { Address To: } \\
\begin{array}{l}\text { DOE-HQ } \\
\quad \text { DOE-RL } \\
\\
\text { Other (Specify) }\end{array}\end{array}$ \\
\hline \multicolumn{4}{|c|}{ Description and what constitutes completion of this commitment } \\
\hline \multicolumn{4}{|c|}{$\begin{array}{l}\text { Complete and submit to DOE-RL the "WHC Year End ALARA Goal Status Report" } \\
\text { of calendar year } 1994 \text { sitewide and facility specific ALARA goals. } \\
\text { Commitment is complete following submittal of goal report to DOE-RL }\end{array}$} \\
\hline \multicolumn{2}{|c|}{ Cost Account Manager: Date: } & \multicolumn{2}{|c|}{ Program/Project Manager: Date: } \\
\hline \multicolumn{2}{|l|}{ Activity Manager: } & \multicolumn{2}{|c|}{ DOE Monitor: $\quad$ Date: } \\
\hline
\end{tabular}




\begin{tabular}{|c|c|c|}
\hline $\begin{array}{c}\text { Milestone Description } \\
\text { Sheet }\end{array}$ & $\begin{array}{c}\text { Westinghouse Hanford Company } \\
\text { 6.7.2.4 RadCon SSPP }\end{array}$ & $\begin{array}{c}\text { FY } 1995 \\
\text { SSPP }\end{array}$ \\
\hline
\end{tabular}

\begin{tabular}{|c|c|c|c|}
\hline \multicolumn{2}{|c|}{ Title: Submittal of RPP to DOE } & \multicolumn{2}{|c|}{ Date: August 2, 1994} \\
\hline \multicolumn{2}{|c|}{ Assigned To: SR Johnson } & \multicolumn{2}{|l|}{ CIN: } \\
\hline \multicolumn{2}{|c|}{ WBS Designator: $\quad 6.7 .2 .4$} & \multicolumn{2}{|c|}{ Completion Date: $12 / 31 / 94$} \\
\hline \multicolumn{2}{|c|}{ Number: RAD-95-016 } & \multicolumn{2}{|l|}{ Revision: } \\
\hline $\begin{array}{l}\text { Milestone Class: } \\
\square \quad \text { TPA } \\
\quad \text { DOE } \\
\square \quad \text { CONTRACTOR }\end{array}$ & \multicolumn{2}{|c|}{ 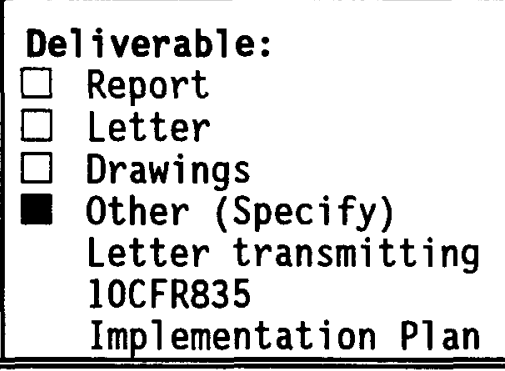 } & $\begin{array}{l}\text { Address To: } \\
\begin{array}{ll} & \text { DOE-HQ } \\
& \text { DOE-RL } \\
& \text { Other (Specify) }\end{array}\end{array}$ \\
\hline \multicolumn{4}{|c|}{ Description and what constitutes completion of this commitment } \\
\hline \multicolumn{4}{|c|}{$\begin{array}{l}\text { Formal submittal to DOE-RL of the WHC Implementation Plan for } 10 \text { CFR } 835 \text {, } \\
\text { including the Radiation Protection Program elements required by } 10 \text { CFR } 835 \text {. } \\
\text { The milestone is complete with the formal transmittal of the WHC approved } \\
\text { implementation plan to DOE-RL. }\end{array}$} \\
\hline \multicolumn{2}{|c|}{ Cost Account Manager: Date: } & \multicolumn{2}{|c|}{ Program/Project Manager: Date: } \\
\hline \multicolumn{2}{|l|}{ Activity Manager: } & \multicolumn{2}{|c|}{ DOE Monitor: $\quad$ Date: } \\
\hline
\end{tabular}




\begin{tabular}{|c|c|c|}
\hline $\begin{array}{c}\text { Milestone Description } \\
\text { Sheet }\end{array}$ & $\begin{array}{l}\text { Westinghouse Hanford Company } \\
\text { 6.7.2.4 Radiological Control }\end{array}$ & $\begin{array}{c}\text { FY } 1995 \\
\text { SSPP }\end{array}$ \\
\hline
\end{tabular}

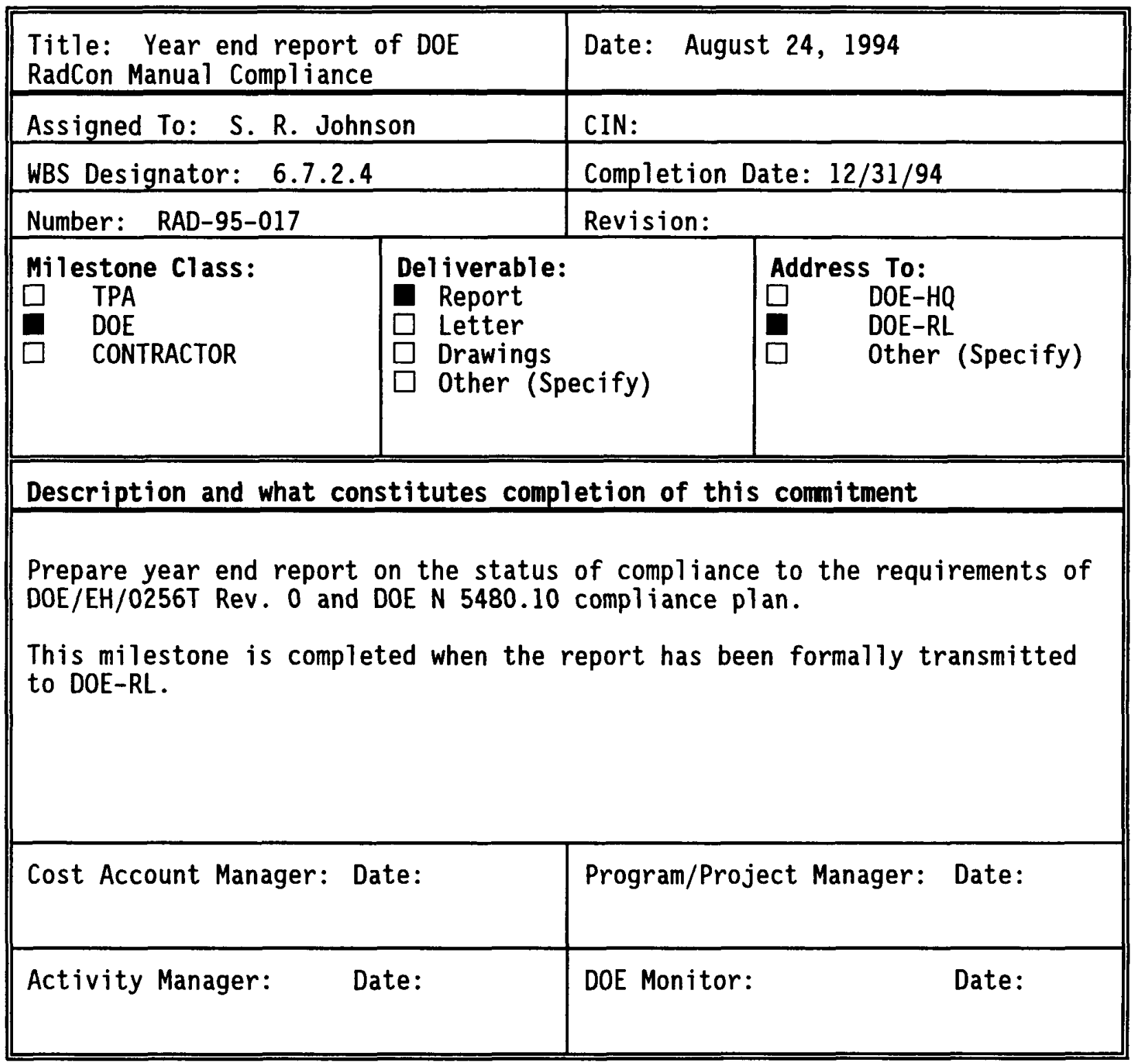




\begin{tabular}{|c|l|c|}
\hline $\begin{array}{c}\text { Milestone Description } \\
\text { Sheet }\end{array}$ & $\begin{array}{l}\text { Westinghouse Hanford Company } \\
6.7 .2 .4 \text { Radiological Control }\end{array}$ & $\begin{array}{c}\text { FY } 1995 \\
\text { SSPP }\end{array}$ \\
\hline
\end{tabular}

\begin{tabular}{|c|c|c|c|}
\hline \multicolumn{2}{|c|}{$\begin{array}{l}\text { Title: Report of Status of HSRCM } \\
\text { Rev 1.0 compliance actions. }\end{array}$} & \multicolumn{2}{|l|}{ Date: August 11, 1994} \\
\hline \multicolumn{2}{|c|}{ Assigned To: SR Johnson } & \multicolumn{2}{|l|}{ CIN: } \\
\hline \multicolumn{2}{|c|}{ WBS Designator: 6.7 .2 .4} & \multicolumn{2}{|l|}{ Completion Date: $9 / 30 / 95$} \\
\hline \multicolumn{2}{|c|}{ Number: $\quad$ RAD-95-018 } & \multicolumn{2}{|l|}{ Revision: } \\
\hline $\begin{array}{l}\text { Milestone Class: } \\
\square \quad \text { TPA } \\
\text { DOE } \\
\square \quad \text { CONTRACTOR }\end{array}$ & \multicolumn{2}{|c|}{$\begin{array}{l}\text { Deliverable: } \\
\square \text { Report } \\
\text { Letter } \\
\square \text { Drawings } \\
\square \text { Other (Specify) }\end{array}$} & $\begin{array}{l}\text { Address To: } \\
\square \quad \text { DOE-HQ } \\
\square \quad \text { DOE-RL } \\
\square \quad \text { Other (Specify) }\end{array}$ \\
\hline \multicolumn{4}{|c|}{ Description and what constitutes completion of this commitment } \\
\hline \multicolumn{4}{|c|}{$\begin{array}{l}\text { This milestone is complete when all FY95 Compliance Actions are completed } \\
\text { and a report for the } 1995 \text { Fiscal Year Compliance Actions for Rev } 1.0 \text { of the } \\
\text { HSRCM is formally transmitted to DOE-RL. }\end{array}$} \\
\hline \multicolumn{2}{|c|}{ Cost Account Manager: Date: } & \multicolumn{2}{|c|}{ Program/Project Manager: Date: } \\
\hline \multicolumn{2}{|l|}{ Activity Manager: } & \multicolumn{2}{|c|}{ DOE Monitor: Date: } \\
\hline
\end{tabular}




\begin{tabular}{|c|c|c|}
\hline $\begin{array}{c}\text { Milestone Description } \\
\text { Sheet }\end{array}$ & $\begin{array}{c}\text { Westinghouse Hanford Company } \\
6.7 .2 .4 \text { Radiological Control }\end{array}$ & $\begin{array}{c}\text { FY } 1995 \\
\text { SSPP }\end{array}$ \\
\hline
\end{tabular}

\begin{tabular}{|c|c|c|c|}
\hline \multicolumn{2}{|c|}{$\begin{array}{l}\text { Title: Complete al1 actions for Rev } \\
0 \text { of the DOE RadCon Manual }\end{array}$} & \multicolumn{2}{|c|}{ Date: } \\
\hline \multicolumn{2}{|c|}{ Assigned To: S. R. Johnson } & \multicolumn{2}{|l|}{ CIN: } \\
\hline \multicolumn{2}{|l|}{ WBS Designator: $\quad 6.7 .2 .4$} & \multicolumn{2}{|c|}{ Completion Date: $3 / 30 / 95$} \\
\hline \multicolumn{2}{|l|}{ Number: RAD-94-019 } & \multicolumn{2}{|c|}{ Revision: } \\
\hline 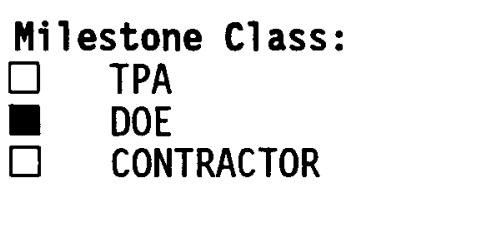 & \multicolumn{2}{|c|}{$\begin{array}{l}\text { Deliverable: } \\
\square \text { Report } \\
\text { Letter } \\
\square \text { Drawings } \\
\square \text { Other (Specify) }\end{array}$} & 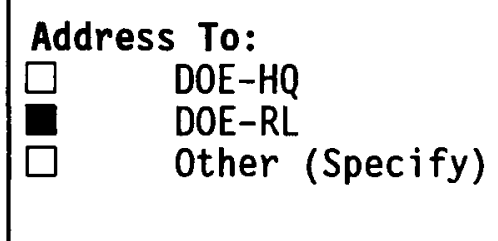 \\
\hline \multicolumn{4}{|c|}{ Description and what constitutes completion of this commitment } \\
\hline \multicolumn{4}{|c|}{$\begin{array}{l}\text { This milestone is complete when all program implementation compliance } \\
\text { actions for DOE/EH/0256T Rev. } 0 \text { are Complete and formally transmitted to } \\
\text { DOE-RL. }\end{array}$} \\
\hline \multicolumn{2}{|c|}{ Cost Account Manager: Date: } & \multicolumn{2}{|c|}{ Program/Project Manager: Date: } \\
\hline Activity Manager: & te: & DOE M & Date: \\
\hline
\end{tabular}




\begin{tabular}{|c|c|c|}
\hline $\begin{array}{c}\text { Milestone Description } \\
\text { Sheet }\end{array}$ & $\begin{array}{c}\text { Westinghouse Hanford Company } \\
6.7 .2 .4 \text { Radiological Control }\end{array}$ & $\begin{array}{c}\text { FY } 1995 \\
\text { SSPP }\end{array}$ \\
\hline
\end{tabular}

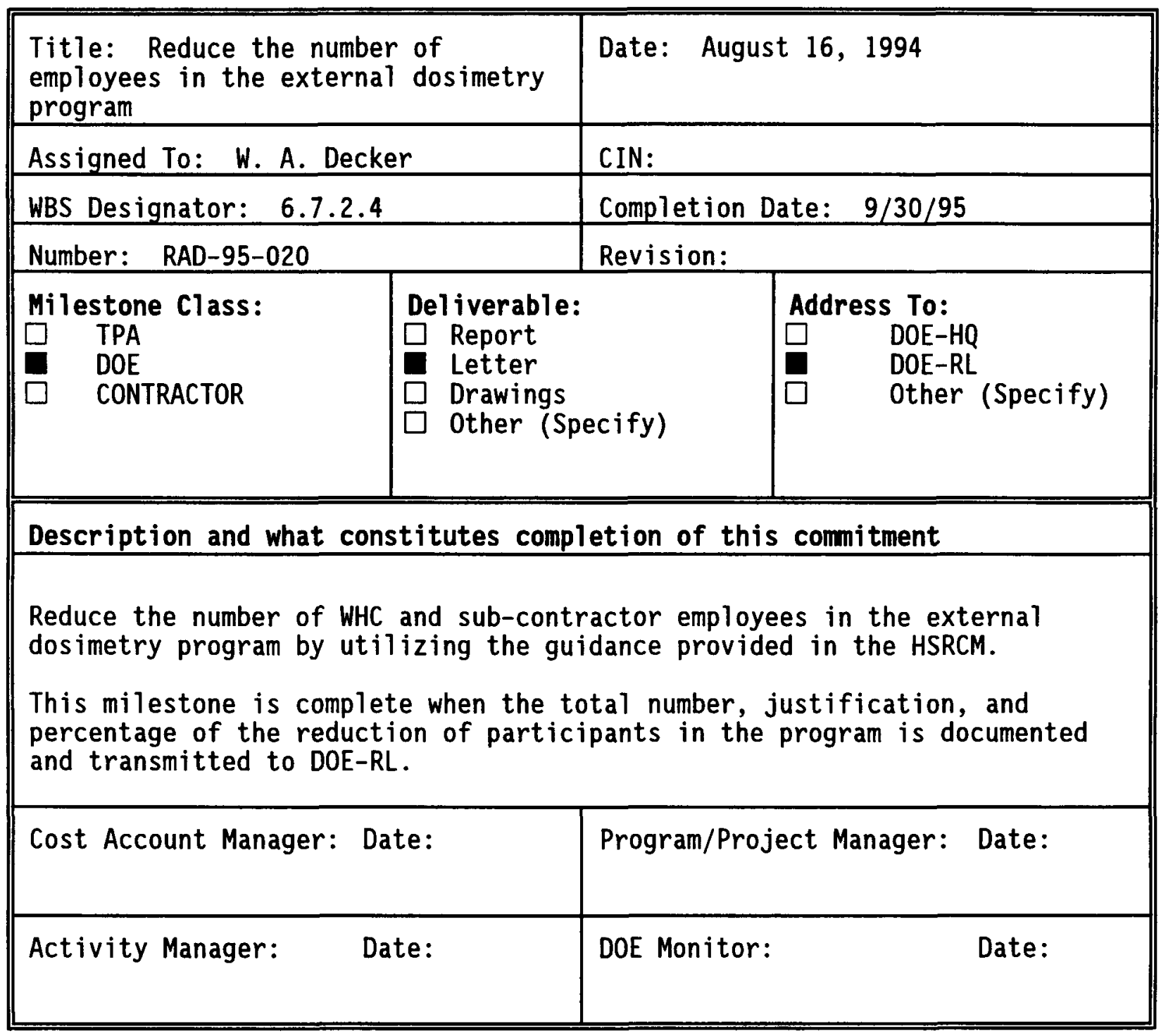




\begin{tabular}{|c|c|c|}
\hline $\begin{array}{c}\text { Milestone Description } \\
\text { Sheet }\end{array}$ & $\begin{array}{c}\text { Westinghouse Hanford Company } \\
6.7 .2 .4 \text { Radiological Control }\end{array}$ & $\begin{array}{c}\text { FY } 1995 \\
\text { SSPP }\end{array}$ \\
\hline
\end{tabular}

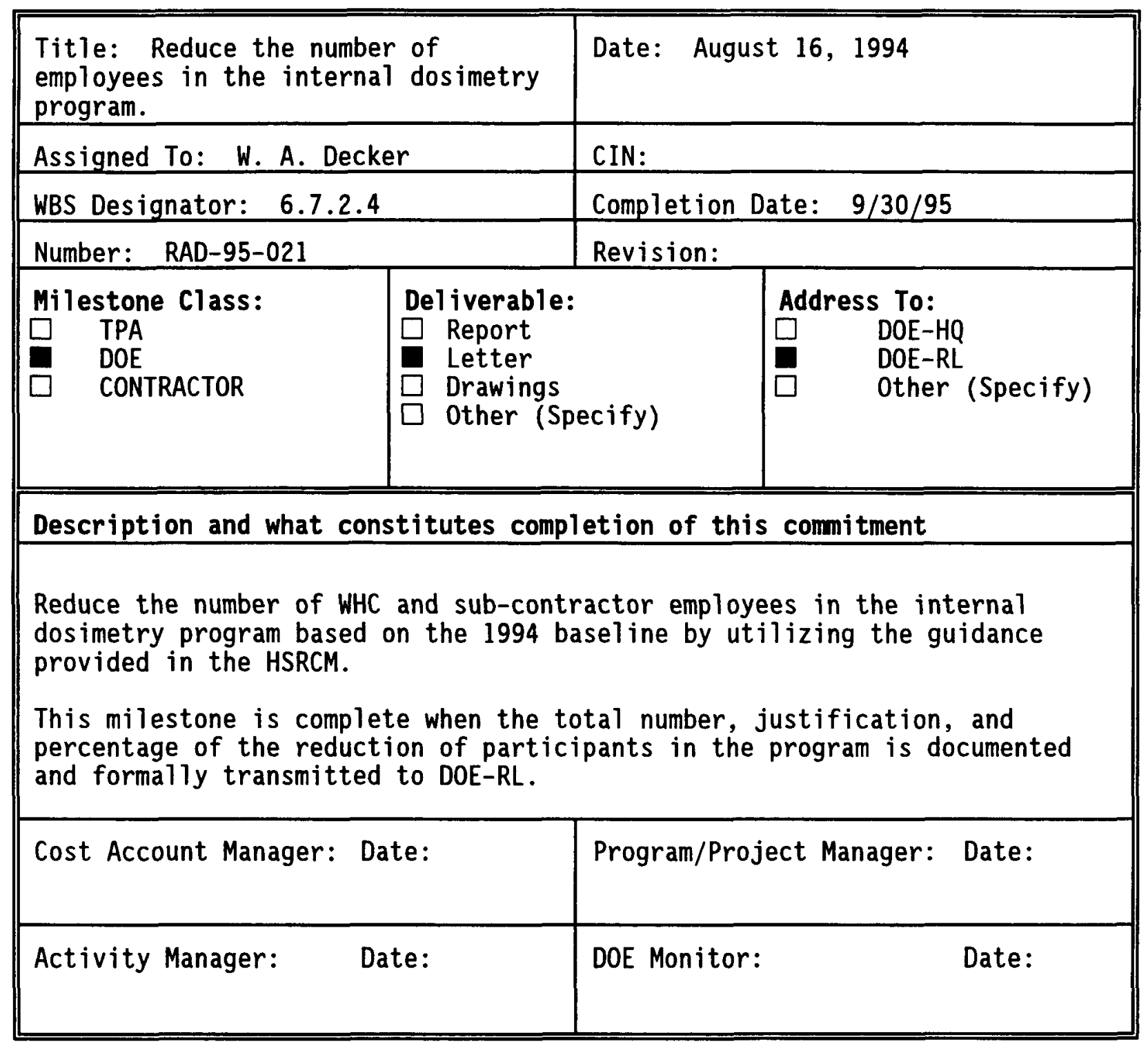




\begin{tabular}{|c|c|c|}
\hline $\begin{array}{c}\text { Milestone Description } \\
\text { Sheet }\end{array}$ & $\begin{array}{l}\text { Westinghouse Hanford Company } \\
6.7 .2 .4 \text { Radiological Control }\end{array}$ & $\begin{array}{c}\text { FY } 1995 \\
\text { SSPP }\end{array}$ \\
\hline
\end{tabular}

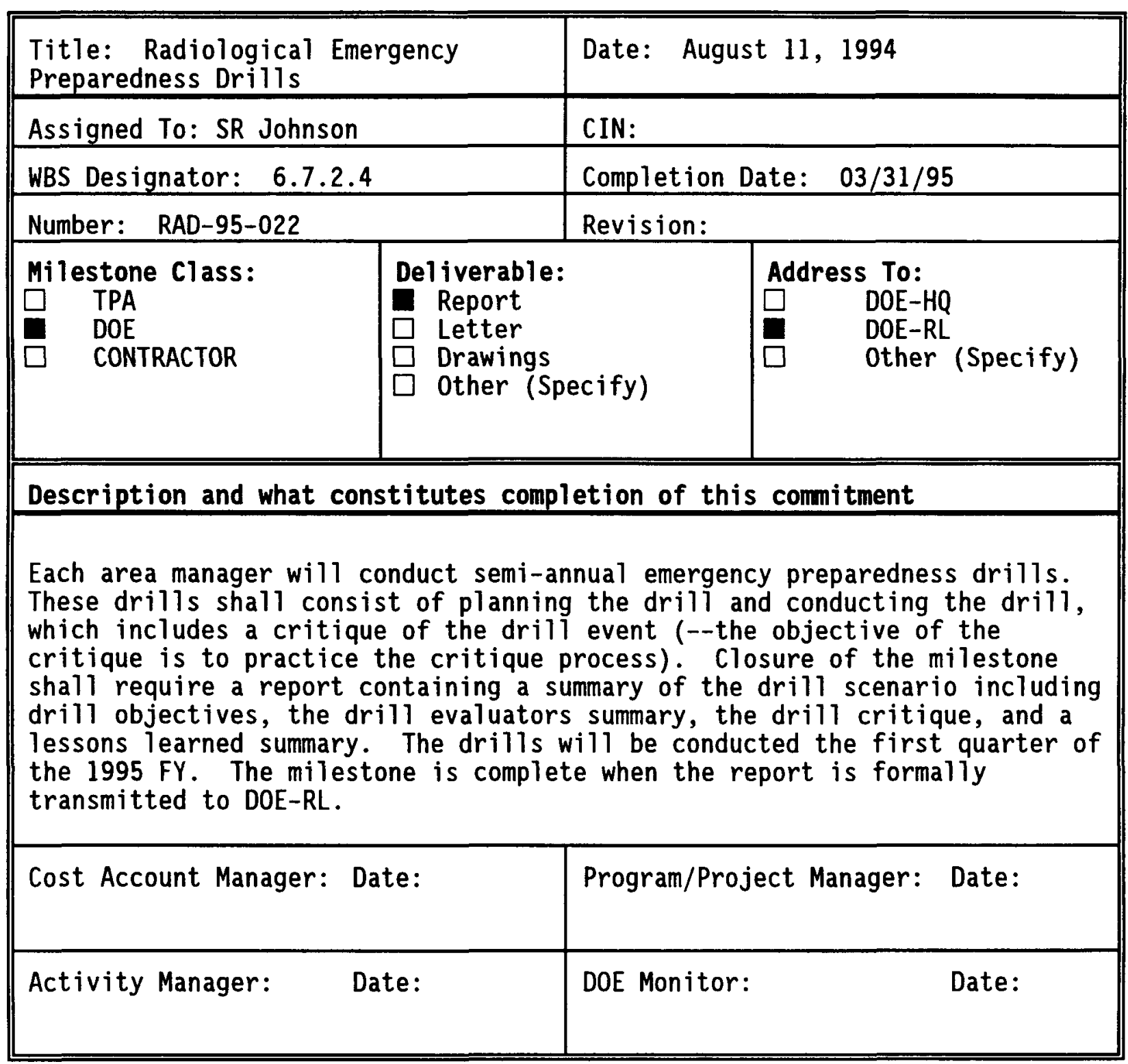




\begin{tabular}{|c|c|c|}
\hline $\begin{array}{c}\text { Milestone Description } \\
\text { Sheet }\end{array}$ & $\begin{array}{c}\text { Westinghouse Hanford Company } \\
6.7 .2 .4 \text { Radiological Control }\end{array}$ & $\begin{array}{c}\text { FY } 1995 \\
\text { SSPP }\end{array}$ \\
\hline
\end{tabular}

\begin{tabular}{|c|c|c|c|}
\hline \multicolumn{2}{|c|}{$\begin{array}{l}\text { Title: Radiological Emergency } \\
\text { Preparedness Drills }\end{array}$} & \multicolumn{2}{|c|}{ Date: August 11, 1994} \\
\hline \multicolumn{2}{|l|}{ Assigned To: SR Johnson } & \multicolumn{2}{|l|}{ CIN: } \\
\hline \multicolumn{2}{|l|}{ WBS Designator: $\quad 6 \cdot 7 \cdot 2.4$} & \multicolumn{2}{|c|}{ Completion Date: $09 / 30 / 95$} \\
\hline \multicolumn{2}{|l|}{ Number: RAD-95-023 } & \multicolumn{2}{|c|}{ Revision: } \\
\hline $\begin{array}{l}\text { Milestone Class: } \\
\square \quad \text { TPA } \\
\quad \text { DOE } \\
\square \quad \text { CONTRACTOR }\end{array}$ & \multicolumn{2}{|c|}{$\begin{array}{l}\text { Deliverable: } \\
\text { Report } \\
\square \text { Letter } \\
\square \text { Drawings } \\
\square \text { Other (Specify) }\end{array}$} & \begin{tabular}{|l} 
Address To: \\
$\square \quad$ DOE-HQ \\
\\
$\begin{array}{l}\text { DOE-RL } \\
\text { Other (Specify) }\end{array}$
\end{tabular} \\
\hline \multicolumn{4}{|c|}{ Description and what constitutes completion of this commitment } \\
\hline \multicolumn{4}{|c|}{$\begin{array}{l}\text { Each area manager will conduct semi-annual emergency preparedness drills, } \\
\text { These drills shall consist of planning the drill and conducting the drili, } \\
\text { which includes a critique of the drill event (--the objective of the } \\
\text { critique is to practice the critique process). Closure of the milestone } \\
\text { shall require a report containing a summary of the drill scenario including } \\
\text { drill objectives, the drill evaluators summary, the drill critique, and a } \\
\text { lessons learned summary. The drills will be conducted the third quarter of } \\
\text { the 1995 FY. The milestone is complete when the report is formally } \\
\text { transmitted to DOE-RL. }\end{array}$} \\
\hline \multicolumn{2}{|c|}{ Cost Account Manager: Date: } & \multicolumn{2}{|c|}{ Program/Project Manager: Date: } \\
\hline Activity Manager: & ate: & DOE Mo & Date: \\
\hline
\end{tabular}




\begin{tabular}{||c|c|c|}
\hline $\begin{array}{c}\text { Milestone Description } \\
\text { Sheet }\end{array}$ & $\begin{array}{c}\text { Westinghouse Hanford Company } \\
\text { 6.7.2.4 Radiological Control }\end{array}$ & $\begin{array}{c}\text { FY } 1995 \\
\text { SSPP }\end{array}$ \\
\hline
\end{tabular}

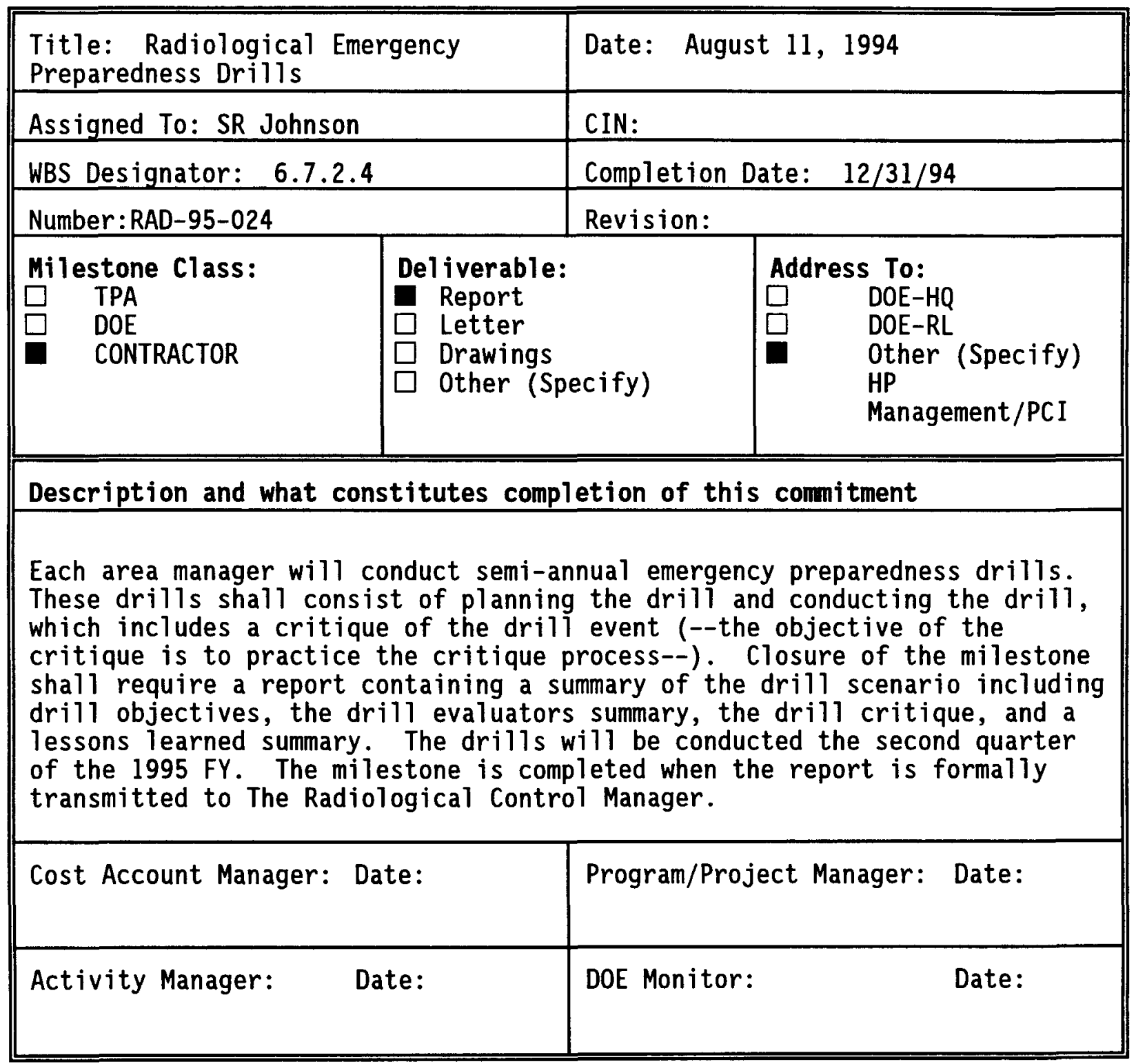




\begin{tabular}{|c|c|c|}
\hline $\begin{array}{c}\text { Milestone Description } \\
\text { Sheet }\end{array}$ & $\begin{array}{l}\text { Westinghouse Hanford Company } \\
\text { 6.7.2.4 Radiological Control }\end{array}$ & $\begin{array}{c}\text { FY } 1995 \\
\text { SSPP }\end{array}$ \\
\hline
\end{tabular}

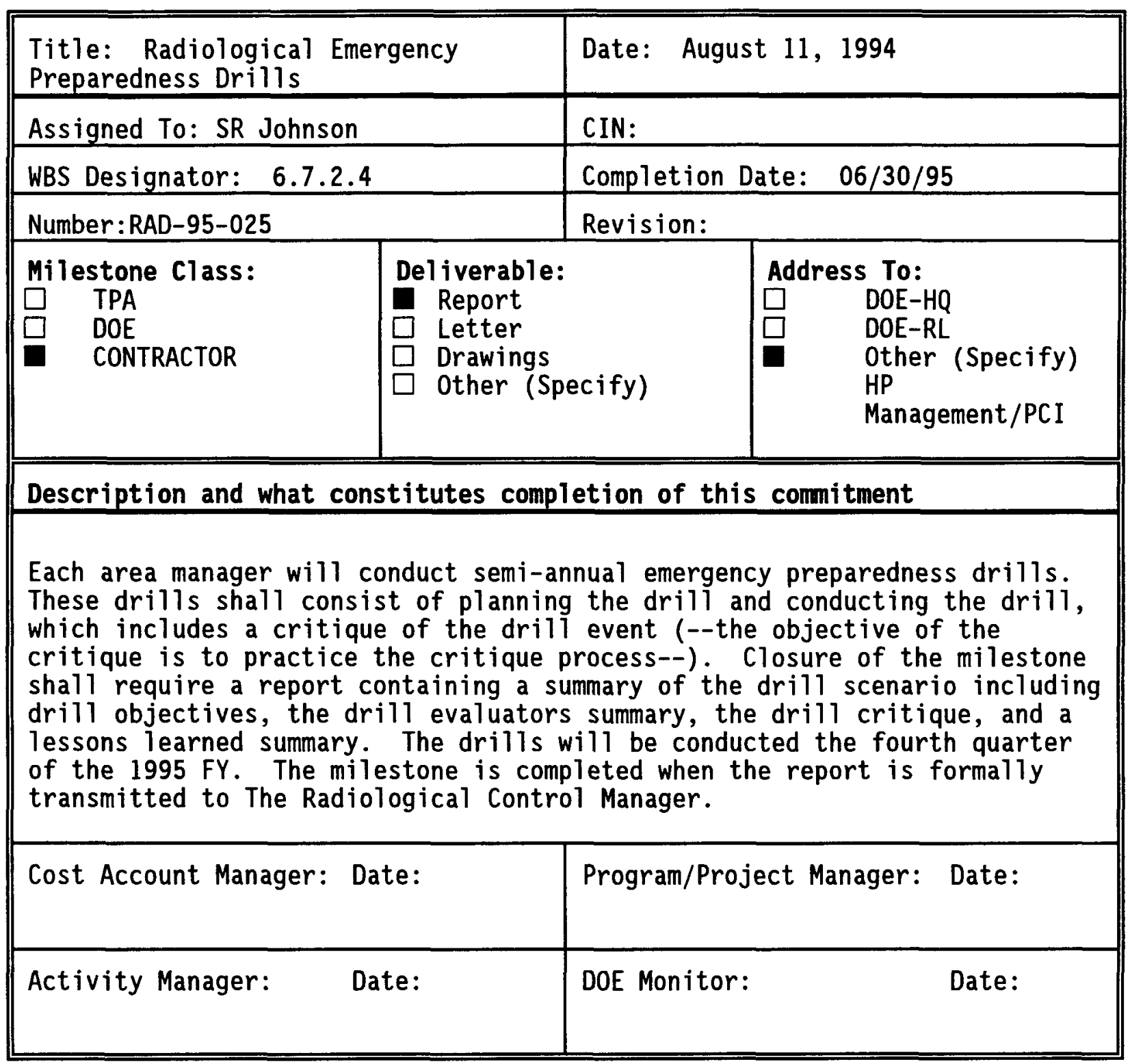




\begin{tabular}{|l|c|c|}
\hline \multicolumn{1}{|c|}{$\begin{array}{c}\text { Radiological Control Direct } \\
\text { Funded Activities }\end{array}$} & $\begin{array}{c}\text { West inghouse Hanford Company } \\
\text { Program Management } \\
\text { Radiological Control Direct Funded } \\
\text { Projects }\end{array}$ & $\begin{array}{c}\text { Fite Support Program Plan } \\
\text { Date Prepared: August 30, 1994 } \\
\begin{array}{l}\text { Direct funded Activities with } \\
\text { RC Programmatic oversight }\end{array}\end{array}$ \\
\hline
\end{tabular}

Direct Program Funding--Solid Waste, T-Plant, and the Effluent Treatment Facility provide funding based on all Radiological Control activities.

\section{Technical Content}

Manage and direct the operation of field Radiological Control organizations providing a broad range of Radiological Controls support and services to WHC plants and operations and selected services on request to DOE-RL, KEH, and other DOE-RL contractors and subcontractors. Monitor, analyze, and report Radiological Control experience/performance and direct or effectively recommend corrective and improvement actions as needed to ensure a radiologically safe and healthy workplace, protect public health and safety, and clearly communicate and demonstrate the strong WHC and DOE commitments to safety and productivity.

Regulatory requirements: DOE/EH-0256T, 10 CFR 835, 10 CFR 834; HSRCM, WHC Control Manuals (WHC-CM-1-6, WHC-CM-4-12, WHC-CM-4-14, and WHC-CM-7-5).

\section{Objectives:}

Support the Safety and Quality Program providing radiological support to facility operation and maintenance activities. These activities consist of administrative support, facility operational and decontamination activity support, preventive and corrective maintenance support, project support, routine and emergency surveillance support, and effluent monitoring support.

For further details of tasks and activities see the project program plan. 


\begin{tabular}{|c|c|c|}
\hline $\begin{array}{c}\text { Radiological Control Direct } \\
\text { Funded Activities } \\
\end{array}$ & $\begin{array}{c}\text { Westinghouse Hanford Company } \\
\text { Program Management }\end{array}$ & $\begin{array}{c}\text { FY } 1995 \\
\text { Site Support Program Plan }\end{array}$ \\
\hline $\begin{array}{l}\text { Cost Account No. } \\
\text { Direct funded Activities with } \\
\text { RC Programmatic oversight }\end{array}$ & $\begin{array}{c}\text { Radiological Control Direct Funded } \\
\text { Projects }\end{array}$ & $\begin{array}{c}\text { Date Prepared: August 30, } 1994 \\
\text { Revision \#0 }\end{array}$ \\
\hline
\end{tabular}

A11 personnel are direct funded; there is no indirect funding for the $K$ Basins Radiological Control program.

The purpose of $K$ Basins Radiological Control is to provide radiation protection services and facility specific oversight to the $K$ Basins. This support is required to assist WHC in accomplishing SNF objectives at the $K$ Basins. Services provided include (but are not 1 imited to):

- Radiological access control for personnel and visitors

- Ensuring that $K$ Basins Radiological Control personnel receive the necessary training and maintain the required qualifications

- Providing radiological release surveys for personnel, equipment, and materials

- Performing required radiological surveillance

- Supporting hazardous waste shipments by providing shipment surveys and sample analysis

- Reviewing technical work documents to ensure sufficient radiological safety steps are incorporated in work instructions

- $\quad$ Preparing radiation work permits

- Reporting, tracking, and trending radiological problems and overall radiological safety performance

- Maintaining and operating, a laboratory for the purpose of supporting most of the $K$ Basins radiological sampling and analysis needs.

This list describes the major elements of the $K$ Basins Radiological Control program. More specific descriptions of the program objectives are found in the $K$ Basins Radiological Control Fiscal Year Work Plan.

All personnel (manager, exempt, nonexempt, and bargaining unit) are funded through direct source. 100 Areas FRC has no indirect funding. 


\begin{tabular}{|l|c|c|}
\hline \multicolumn{1}{|c|}{$\begin{array}{c}\text { Radiological Control Direct } \\
\text { Funded Activities }\end{array}$} & $\begin{array}{c}\text { Westinghouse Hanford Company } \\
\text { Program Management }\end{array}$ & $\begin{array}{c}\text { FY } 1995 \\
\text { Site Support Program Plan } \\
\text { Dadiological Control Direct Funded } \\
\text { Projects }\end{array}$ \\
$\begin{array}{l}\text { Direct funded Activities with } \\
\text { RC Programmatic oversight }\end{array}$ & Radicougust 30, 1994 \\
\hline
\end{tabular}

This work element receives direct funding from Waste Tank Safety \& Operations Program; Tank Waste Disposal Program; Solid/Liquid Waste Remediation Program; EM-30 Site Support Program; and Hanford Waste Vitrification Program.

Costs include personnel, training, site services, and materials associated with these work activities.

Administer and maintain Radiological Control support to Tank Waste Remediation Systems (TWRS) including Element Task Description

See the TWRS program plan document for details. 


\begin{tabular}{|c|c|c|}
\hline $\begin{array}{c}\text { Radiological Control Direct } \\
\text { Funded Activities }\end{array}$ & $\begin{array}{c}\text { Westinghouse Hanford Company } \\
\text { Program Management }\end{array}$ & $\begin{array}{c}\text { FY } 1995 \\
\text { Site Support Program Plan }\end{array}$ \\
\hline $\begin{array}{l}\text { Cost Account No. } \\
\text { Direct funded Activities with } \\
\text { RC Programmatic oversight }\end{array}$ & $\begin{array}{c}\text { Radiological Control Direct Funded } \\
\text { Projects }\end{array}$ & $\begin{array}{c}\text { Date Prepared: August 30, } 1994 \\
\text { Revision \#0 }\end{array}$ \\
\hline
\end{tabular}

\section{PUREX/U03}

Direct funded program--Manage and direct the operation of Field Radiological Control at PUREX and U03, providing a broad range of Radiological Control support and services to WHC operations and other selected services on request to DOE-RL, KEH and DOE-RL contractors and subcontractors. Monitor, analyze and report Radiological Control experience/performance and direct or effectively recommend corrective and improvement actions as needed to ensure a radiologically safe and healthy workplace, protect public

health and safety, and clearly communicate and demonstrate the strong WHC and DOE commitments to safety with productivity. Regulatory divers include DOE/EH-0256T, HSRCM and WHC-CM-1-6.

- Maintain a trained and qualified Radiological Control staff to meet program objectives and customer needs.

- Provide a scheduled routine radiological surveillance program.

- Identify and report abnormal radiological conditions and provide facility management with technically based recommended corrective actions.

- Provide emergency response capability to radiological accidents/events.

- Provide a cost effective radiological control program which complies with laws, regulations, and DOE orders and meets program objectives.

- The implementation of DOE/EH-0256T, HSRCM and WHC-CM-1-6, will be given high priority.

- U03 cleanup will be complete and will require a minimum staff for surveillance.

No new requirements will be imposed.

For further detail see the program plan for the project. 


\begin{tabular}{|l|c|c|}
\hline \multicolumn{1}{|c|}{$\begin{array}{c}\text { Radiological Control Direct } \\
\text { Funded Activities }\end{array}$} & $\begin{array}{c}\text { Westinghouse Hanford Company } \\
\text { Program Management }\end{array}$ & $\begin{array}{c}\text { FY 1995 } \\
\text { Site Support Program Plan } \\
\text { Dadiological Control Direct Funded } \\
\text { Projects }\end{array}$ \\
$\begin{array}{l}\text { Direct funded Activities with } \\
\text { RC Programmatic oversight }\end{array}$ & & Revision \#0 \\
\hline
\end{tabular}

\section{PFP/Laboratories}

Direct funded program funding. Al1 personnel (manager, exempt, nonexempt, and bargaining unit) are funded through direct source.

Administer and maintain Health Physics Technician (HPT) support to PFP/Laboratory. Provide HPT coverage for the Access Control Entry System (ACES), required training, final personnel exit surveys, and required radiation routines in accordance with the appropriate DOE Orders and WHC manuals. PFP/Laboratory Radiological Control will ensure that all personnel entering a radiation area have met the entry requirements per DOE/EH-0256T, HSRCM, and WHC-CM-1-6. Provide OSR surveillance for environmental effluents to ensure compliance with PFP Safety Analysis Report, SAR-021 and 10 CFR 835, as implemented.

\section{Objectives}

Provide HPT support for routines, surveillances, WRAM coverage, training, lab support, 2736-ZB support, surveillance and routines of gaseous and liquid effluents, project construction, PMs and Pisces, and Maintenance and Operations activities.

\section{Element Task Description}

\section{Cost Content}

All activities and tasks are direct funded from PUREX and U03 Programs.

See the project program plans for specific details and tasks of direct funded activities. 


\begin{tabular}{|c|c|c|}
\hline $\begin{array}{c}\text { Radiological Control Direct } \\
\text { Funded Activities }\end{array}$ & $\begin{array}{c}\text { Westinghouse Hanford Company } \\
\text { Program Management }\end{array}$ & $\begin{array}{c}\text { FY } 1995 \\
\text { Site Support Program Plan }\end{array}$ \\
\hline $\begin{array}{l}\text { Cost Account No. } \\
\text { Direct funded Activities with } \\
\text { RC Programmatic oversight }\end{array}$ & $\begin{array}{c}\text { Radiological Control Direct Funded } \\
\text { Projects }\end{array}$ & $\begin{array}{c}\text { Date Prepared: August 30, } 1994 \\
\text { Revision \#0 }\end{array}$ \\
\hline
\end{tabular}

\section{All activities and tasks are direct funded from B Plant and Waste Encapsulation Storage Facility (WESF)} Programs.

\section{Technical Content}

Manage and direct the operation of field Radiological Control at B Plant and WESF, providing a broad range of Radiological Control support and services to WHC operations and other selected services, on request, to DOE-RL, KEH, and other DOE-RL contractors and subcontractors. Monitor, analyze, and report Radiological Control experience/performance and direct or effectively recommend corrective and improvement actions as needed to ensure a radiologically safe and healthy workplace, protect public health and safety, and clearly communicate and demonstrate the strong WHC and DOE commitments to safety with productivity. Regulatory drivers include DOE/EH-0256T, HSRCM and WHC-CM-1-6.

\section{Objectives}

- Implement an effective radiological survey and release program that meets DOE and WHC requirements.

- Support implementation of 10 CFR 835 and the HSRCM policies and guidelines.

- Support development and maintenance of WHC-IP-0718 procedures.

- Assure appropriate budget is defined and implemented as approved within MCS/FDS to support approved work scope.

- Provide a document safety review capability.

For further task detail see the Project Plan. 


\begin{tabular}{|l|c|c|}
\hline \multicolumn{1}{|c|}{$\begin{array}{c}\text { Radiological Control Direct } \\
\text { Funded Activities }\end{array}$} & $\begin{array}{c}\text { Westinghouse Hanford Company } \\
\text { Program Management } \\
\text { Radiological Control Direct Funded } \\
\text { Post Account No. }\end{array}$ & $\begin{array}{c}\text { Fy } 1995 \\
\text { Pite Support Program Plan } \\
\text { Direct funded Activities with }\end{array}$ \\
RC Programmatic oversight & & Revision \#0 \\
\hline
\end{tabular}

Radiological Control Training provides the training for Health Physics Technicians (HPTs) on a bill back basis. The customer is charged a rate for each training module. Training is provide for all levels of HPT training and Radiological worker training.

Specific training details can be found in the program plan for Technical Training. 
FIGURE 1

\begin{tabular}{|c|c|c|c|c|c|c|}
\hline \multicolumn{7}{|c|}{ ESQ FY 1995 Aligned Staff - Transition Projects } \\
\hline & $\begin{array}{c}\text { SAS } \\
(3 B 000)\end{array}$ & $\begin{array}{c}\text { HFD } \\
(3 \mathrm{COOO})\end{array}$ & $\begin{array}{l}\text { Safety } \\
(31000)\end{array}$ & $\begin{array}{c}\text { Radiological } \\
\text { Control } \\
(33000)\end{array}$ & $\begin{array}{c}Q A \\
(38000)\end{array}$ & TOTAL \\
\hline \multicolumn{7}{|c|}{ Program of Projects } \\
\hline TRP General & 10.3 & & & & & 10.3 \\
\hline PFP & 125.0 & 6.0 & 11.0 & 48.0 & 17.5 & 207.5 \\
\hline B Plant & & 1.0 & 4.5 & 22.0 & 4.6 & 32.1 \\
\hline PUREX & 1.0 & 2.0 & 5.5 & 34.0 & 6.6 & 49.1 \\
\hline FFTF & 21.0 & & 10.5 & 12.0 & 10.1 & 53.6 \\
\hline FMEF & & 1.0 & & & & 1.0 \\
\hline 300 Area & 2.4 & 1.0 & 2.0 & & 0.3 & 5.7 \\
\hline TBD & & & & 7.0 & & 7.0 \\
\hline Total & 159.7 & 11.0 & 33.5 & 123.0 & 39.1 & 366.3 \\
\hline
\end{tabular}


FIGURE 2

\begin{tabular}{|c|c|c|c|c|c|c|}
\hline \multicolumn{7}{|c|}{ ESQ FY 1995 Aligned Staff - Spent Nuclear Fuel Project } \\
\hline & $\begin{array}{c}\text { SAS } \\
(3 \mathrm{~B} 000)\end{array}$ & $\begin{array}{c}\text { HFD } \\
(3 \mathrm{COO0})\end{array}$ & $\begin{array}{c}\text { Safety } \\
(31000)\end{array}$ & $\begin{array}{c}\text { Radiological } \\
\text { Control } \\
(33000) \\
\end{array}$ & $\begin{array}{c}\text { QA } \\
(38000)\end{array}$ & TOTAL \\
\hline \multicolumn{7}{|c|}{ Spent Nuclear Fuel Project } \\
\hline K-BASINS & & & & 35.0 & & \\
\hline & & & & & & \\
\hline & & & & & & \\
\hline & & & & & & \\
\hline & & & & & & \\
\hline & & & & & & \\
\hline Total & 24.0 & 2.0 & 13.0 & 35.0 & 10.0 & 84.0 \\
\hline
\end{tabular}


FIGURE 3

\begin{tabular}{|c|c|c|c|c|c|c|}
\hline \multicolumn{7}{|c|}{ ESQ FY 1995 Aligned Staff - TWRS } \\
\hline & $\begin{array}{c}\text { SAS } \\
(3 B 000)\end{array}$ & $\begin{array}{c}\text { HFD } \\
(3 \mathrm{COO0})\end{array}$ & $\begin{array}{c}\text { Safety } \\
(31000)\end{array}$ & $\begin{array}{c}\text { Radiological } \\
\text { Control } \\
(33000) \\
\end{array}$ & $\begin{array}{c}\text { QA } \\
(38000)\end{array}$ & TOTAL \\
\hline \multicolumn{7}{|l|}{ TWRS } \\
\hline \multicolumn{7}{|l|}{ TWRS Plant } \\
\hline West Tank Farm & & & & 39.0 & & \\
\hline East Tank Farm/EVAP & & & & 41.0 & & \\
\hline Compliance Sampling & & & & 24.0 & & \\
\hline Mgmnt Support & & & & 5.0 & & \\
\hline Tech Support & & & & 14.0 & & \\
\hline Total & 5.0 & 4.0 & 63.5 & 123.0 & 72.0 & 267.5 \\
\hline
\end{tabular}




\section{FIGURE 4}

\begin{tabular}{|c|c|c|c|c|c|c|}
\hline \multicolumn{7}{|c|}{ ESQ FY 1995 Aligned Staff - HAE } \\
\hline & $\begin{array}{c}\text { SAS } \\
(3 \mathrm{BO00})\end{array}$ & $\begin{array}{c}\text { HFD } \\
(3 \mathrm{COO0})\end{array}$ & $\begin{array}{l}\text { Safety } \\
(31000)\end{array}$ & $\begin{array}{c}\text { Radiological } \\
\text { Control } \\
(33000) \\
\end{array}$ & $\begin{array}{c}\text { QA } \\
(38000)\end{array}$ & TOTAL \\
\hline \multicolumn{7}{|l|}{ WAE } \\
\hline \multicolumn{7}{|l|}{$\begin{array}{l}\text { Site Investigation/ } \\
\text { Interim Staff }\end{array}$} \\
\hline \multicolumn{7}{|l|}{ Field Support } \\
\hline \multicolumn{7}{|l|}{$\begin{array}{l}N \text { Reactor } \\
\text { Deactivation }\end{array}$} \\
\hline \multicolumn{7}{|l|}{ Inactive Facilities } \\
\hline T Plant & & & & 25.6 & & \\
\hline Solid Waste & & & & 17.6 & & \\
\hline $\begin{array}{l}200 \text { Area Liquid } \\
\text { Effluent }\end{array}$ & & & & 10.8 & & \\
\hline \multicolumn{7}{|l|}{100 Area Facilities } \\
\hline $\begin{array}{l}222 S \text { Laboratory \& } \\
\text { HSCF }\end{array}$ & & & & 27.0 & & \\
\hline Total & 0.0 & 1.0 & 22.5 & 81.0 & 43.0 & 147.5 \\
\hline
\end{tabular}


FIGURE 5

\begin{tabular}{|c|c|c|c|c|c|c|}
\hline \multicolumn{7}{|c|}{ ESQ FY 1995 Aligned Staff - Landlord } \\
\hline & $\begin{array}{c}\text { SAS } \\
(3 B 000)\end{array}$ & $\begin{array}{c}\text { HFD } \\
(3 \mathrm{COO0})\end{array}$ & $\begin{array}{c}\text { Safety } \\
(31000)\end{array}$ & $\begin{array}{l}\text { Radiological } \\
\text { Control } \\
(33000)\end{array}$ & $\begin{array}{c}\text { QA } \\
(38000)\end{array}$ & TOTAL \\
\hline \multicolumn{7}{|l|}{ Landlord } \\
\hline KEH LANDLORD ACTIVITY & & & & 6.0 & & \\
\hline $\begin{array}{l}\text { KEH VEHICLE } \\
\text { MAINTENANCE }\end{array}$ & & & & 6.0 & & \\
\hline & & & & & & \\
\hline & & & & & & \\
\hline & & & & & & \\
\hline & & & & & & \\
\hline Total & 0.0 & 13.0 & 2.0 & 12.0 & 0.0 & 27.0 \\
\hline
\end{tabular}

